\title{
Mission Design, Guidance, and Navigation of a Callisto-lo- Ganymede Triple Flyby Jovian Capture
}

Alan M. Didion

Follow this and additional works at: https://researchrepository.wvu.edu/etd

\section{Recommended Citation}

Didion, Alan M., "Mission Design, Guidance, and Navigation of a Callisto-lo-Ganymede Triple Flyby Jovian Capture" (2015). Graduate Theses, Dissertations, and Problem Reports. 5495.

https://researchrepository.wvu.edu/etd/5495

This Thesis is protected by copyright and/or related rights. It has been brought to you by the The Research Repository @ WVU with permission from the rights-holder(s). You are free to use this Thesis in any way that is permitted by the copyright and related rights legislation that applies to your use. For other uses you must obtain permission from the rights-holder(s) directly, unless additional rights are indicated by a Creative Commons license in the record and/ or on the work itself. This Thesis has been accepted for inclusion in WVU Graduate Theses, Dissertations, and Problem Reports collection by an authorized administrator of The Research Repository @ WVU. For more information, please contact researchrepository@mail.wvu.edu. 


\title{
Mission Design, Guidance, and Navigation of a Callisto-Io- Ganymede Triple Flyby Jovian Capture
}

\author{
Alan M. Didion \\ Thesis submitted \\ to the Statler College of Engineering \& Mineral Resources \\ at West Virginia University \\ in partial fulfillment of the requirements for the degree of \\ Master of Science in \\ Aerospace Engineering
}

Committee:

Alfred Lynam, Ph.D., Chair

John Christian, Ph.D.

Jason Gross, Ph.D.

Department of Mechanical \& Aerospace Engineering

Morgantown, West Virginia

2015

Keywords: Astrodynamics, Orbital Mechanics, Aerospace, Triple Flyby, Jupiter Copyright 2015 Alan M. Didion 


\title{
ABSTRACT \\ Mission Design, Guidance, and Navigation of a Callisto-Io- Ganymede Triple Flyby Jovian Capture
}

\author{
by Alan M. Didion
}

Use of a triple-satellite-aided capture maneuver to enter Jovian orbit reduces insertion $\Delta \mathrm{V}$ and provides close flyby science opportunities at three of Jupiter's four large Galilean moons. This capture can be performed while maintaining appropriate Jupiter standoff distance and setting up a suitable apojove for plotting an extended tour.

This paper has three main chapters, the first of which discusses the design and optimization of a triple-flyby capture trajectory. A novel triple-satellite-aided capture uses sequential flybys of Callisto, Io, and Ganymede to reduce the $\Delta \mathrm{V}$ required to capture into orbit about Jupiter. An optimal broken-plane maneuver is added between Earth and Jupiter to form a complete chemical/impulsive interplanetary trajectory from Earth to Jupiter. Such a trajectory can yield significant fuel savings over single and double-flyby capture schemes while maintaining a brief and simple interplanetary transfer phase.

The second chapter focuses on the guidance and navigation of such trajectories in the presence of spacecraft navigation errors, ephemeris errors, and maneuver execution errors. A powered-flyby trajectory correction maneuver (TCM) is added to the nominal trajectory at Callisto and the nominal Jupiter orbit insertion (JOI) maneuver is modified to both complete the capture and target the Ganymede flyby. A third TCM is employed after all the flybys to act as a JOI cleanup maneuver. A Monte Carlo simulation shows that the statistical $\Delta \mathrm{V}$ required to correct the trajectory is quite manageable and the flyby characteristics are very consistent. The developed methods maintain flexibility for adaptation to similar launch, cruise, and capture conditions.

The third chapter details the methodology and results behind a completely separate project to design and optimize an Earth-orbiting three satellite constellation to perform very long baseline interferometry (VLBI) as part of the $8^{\text {th }}$ annual Global Trajectory Optimisation Competition (GTOC8). A script is designed to simulate the prescribed constellation and record its observations; the observations made are scored according to a provided performance index. 


\section{Acknowledgements}

The author would like to sincerely thank the faculty and staff of West Virginia University, specifically the Mechanical and Aerospace Engineering Department of the Benjamin M. Statler College of Engineering and Mineral Resources for their guidance, support, and confidence. Furthermore, the author thanks the NASA West Virginia Space Grant Consortium and its staff for its immense support through various periods of the author's educational career up to and including parts of the graduate research described herein. And many thanks to Dr. Alfred Lynam, whose original research provided the foundation of the work presented here, whose guidance shaped its completion, and whose experience ensured that the author make the most of this research project through professional development and publication.

Special thanks to the American Institute of Aeronautics and Astronautics (AIAA) and the American Astronautical Society (AAS), at whose conferences the material for Chapter 1 (San Diego, CA, Aug. '14) and Chapter 2 (Vail, CO, Aug. '15) was presented and peer reviewed. The resulting suggestions and corrections resulted in the publication of Chapter 1's material in the AIAA Journal of Spacecraft and Rockets, and the prospective publication of Chapter 2's material in the journal Acta Astronautica.

Further thanks to the authors and participants involved in the $8^{\text {th }}$ annual Global Trajectory Optimisation Competition (GTOC8): an intense, month-long competition which provided the prompt to generate the material making up Chapter 3 of this manuscript. Specific thanks to Anastassios Petropoulos of NASA JPL, who authored the prompt for the 2015 competition.

Finally, the author expresses deep personal gratitude to family and friends, old and new. It is through the tremendous kindness, support, and confidence of these individuals that the author 
has managed to overcome personal hardship and physical disability to finish his graduate education and author this manuscript in a timely manner. 


\section{Table of Contents}

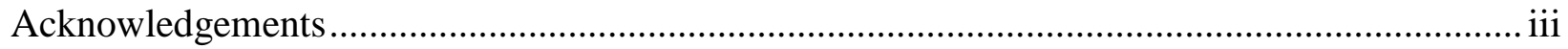

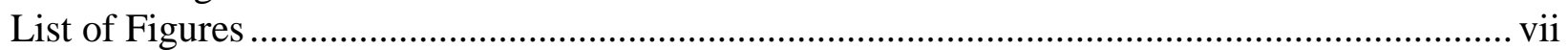

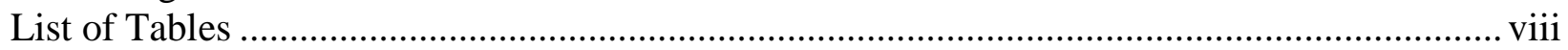

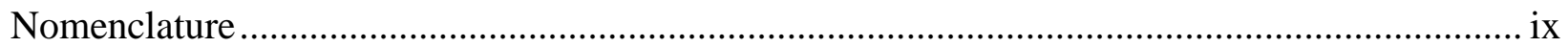

Mission Design, Guidance, and Navigation of a Callisto-Io-Ganymede Triple Flyby Jovian Capture 1

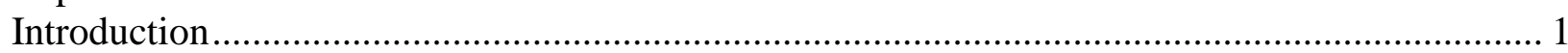

Chapter 1: Design of an Impulsive Trajectory from Earth to Triple Flyby Jovian Capture .......... 4

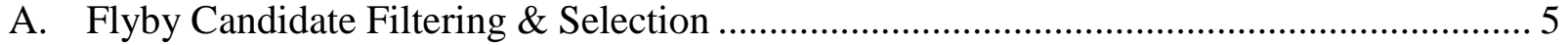

1. Candidate Selection Process....................................................................................... 5

2. Candidate Description ..................................................................................... 6

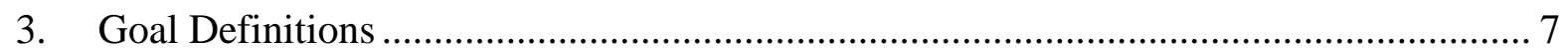

B. Trajectory Design \& Optimization in GMAT …...................................................... 9

1. Software Configuration and Setup ..................................................................... 9

2. Definition of Flyby Coordinate System ............................................................. 11

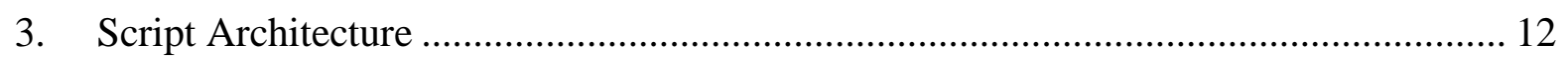

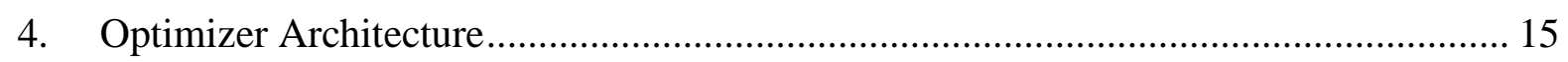

5. Launch Vehicle Considerations .......................................................................... 17

C. Results of GMAT Trajectory Optimization ................................................................. 18

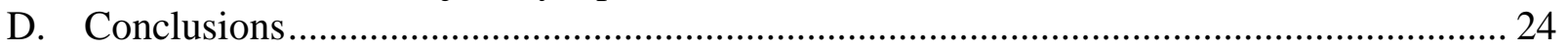

Chapter 2: Navigation and Guidance of a Triple Flyby Jovian Capture...................................... 26

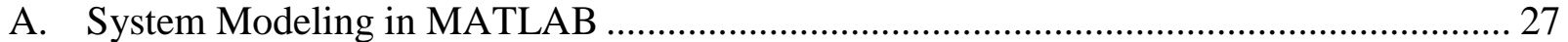

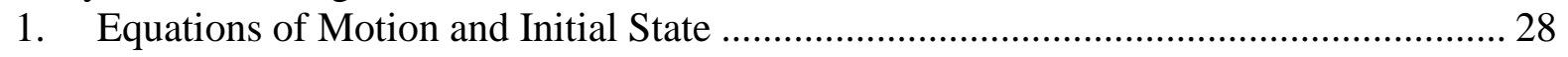

2. The State Transition Matrix ................................................................................... 29

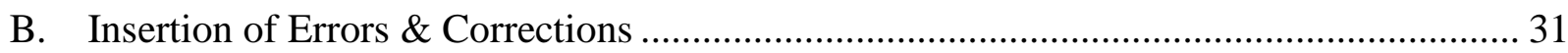

1. State Errors, Ephemeris Errors, and Delta-DOR .............................................. 31

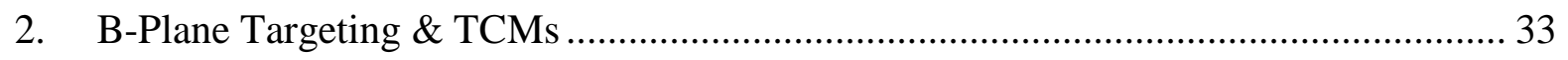

3. Maneuver Execution Error Modeling ..................................................................... 35

C. Statistical Modeling via Monte Carlo Simulation ...................................................... 37

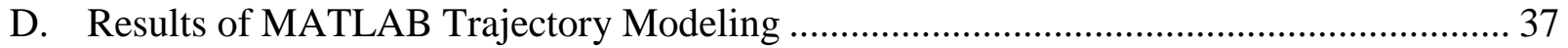

1. Verification of the MATLAB Model \& Nominal Characteristics ................................ 38

2. Monte Carlo Statistical Characteristics .......................................................................... 38

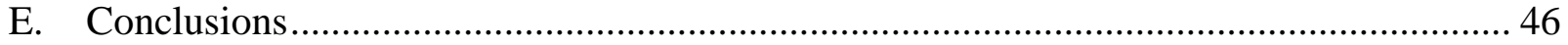

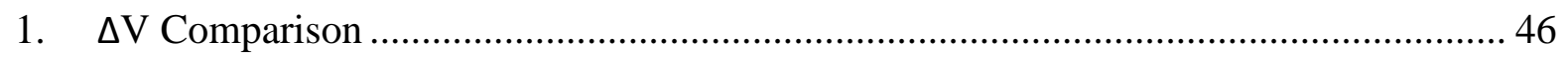

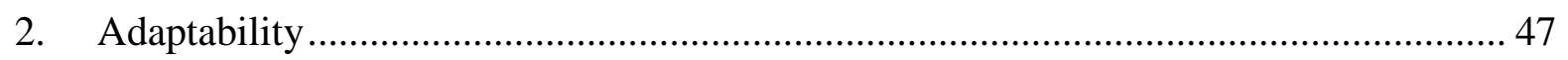

Chapter 3: $8^{\text {th }}$ Annual Global Trajectory Optimisation Competition ........................................ 48

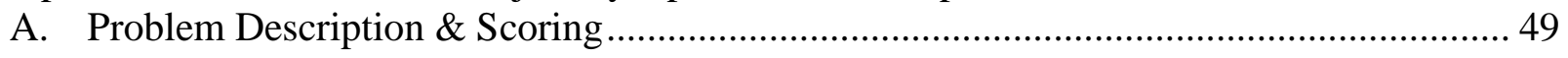

B. Given Conditions and Dynamical Model .......................................................... 52 


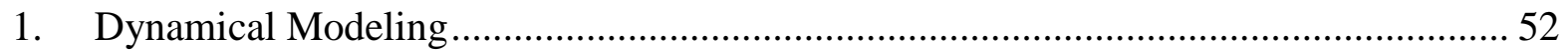

2. Patched-Conics for Lunar Flybys ...................................................................... 54

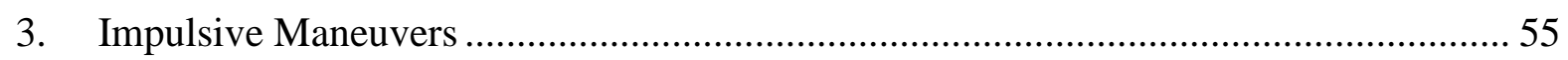

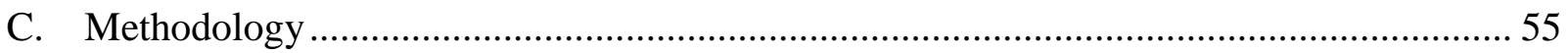

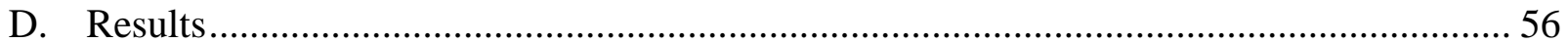

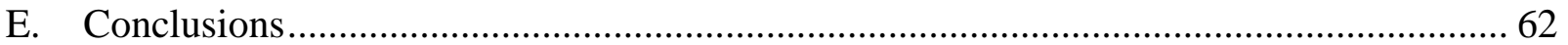

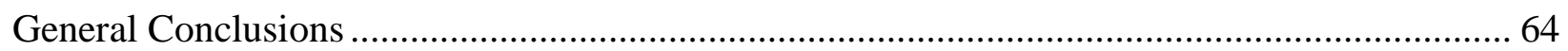

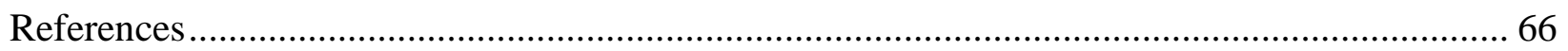




\section{List of Figures}

Figure 1: Basic Diagram of a Triple-Satellite-Aided Capture ............................................... 5

Figure 2: Definition of the B-plane Coordinate System ................................................... 12

Figure 3: Triple Flyby "Nested Solver" Flow Chart ........................................................... 14

Figure 4: Triple Flyby Trajectory Optimization Flow Chart ............................................... 16

Figure 5: Interplanetary Trajectory Optimization Flow Chart................................................. 16

Figure 6: SLS Payload vs. C3 Capabilities [32] .................................................................. 18

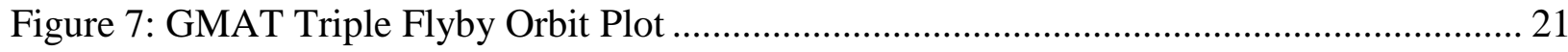

Figure 8: GMAT Plots for the Callisto, Io, and Ganymede Flybys Respectively ...................... 21

Figure 9: GMAT Plot of the Interplanetary Transfer and Earth Escape .................................. 23

Figure 10: Definition of the B-plane Dot Products.......................................................... 28

Figure 11: Example Delta-DOR Setup ..................................................................................... 33

Figure 12: Example Callisto-Io-Ganymede Trajectory, Jupiter-Centered Coordinates ............... 39

Figure 13: Example Callisto-Io-Ganymede Trajectory, Jupiter-Centered Coordinates, Zoomed 40

Figure 14: Callisto B-Plane Scatter, with 1- $\sigma$ and 3- $\sigma$ Error Ellipses.................................... 41

Figure 15: Io B-plane Scatter, with 1- $\sigma$ and 3- $\sigma$ Error Ellipses ........................................... 42

Figure 16: Ganymede B-plane Scatter, with 1- $\sigma$ and $3-\sigma$ Error Ellipses ................................... 43

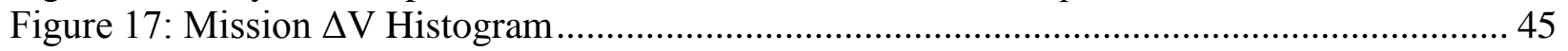

Figure 18: Diagram of an example VLBI Measurement ...................................................... 50

Figure 19: Geocentric X-Y Plot of Initial Constellation Architecture ..................................... 57

Figure 20: Geocentric Y-Z Plot of Initial Constellation Architecture ...................................... 57

Figure 21: Geocentric X-Y Plot of Lunar Rendezvous, Zoomed to the Moon's Orbit ................ 58

Figure 22: Selenocentric X-Y Plot of Lunar Rendezvous ...................................................... 59

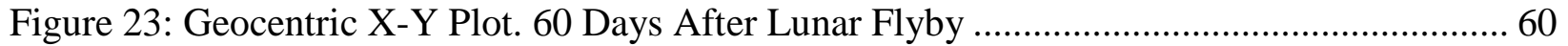

Figure 24: Geocentric X-Z Plot, 60 Days After Lunar Flyby ................................................. 60 


\section{List of Tables}

Table 1: Initial Guess for Jupiter-Centered Cartesian Elements........................................... 7

Table 2: Initial Guess for Jupiter-Centered Keplerian Elements ................................................ 7

Table 3: Altitude and $\Delta \mathrm{V}$ Targets for a Desirable Trajectory ............................................... 8

Table 4: Limits and Recommendations for a Desirable Trajectory ......................................... 8

Table 5: Physical Parameters of the Galilean Moons ................................................................. 10

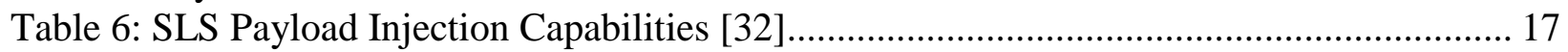

Table 7: Targeted Initial Jupiter-Centered Cartesian Elements for Triple Flyby ........................ 19

Table 8: Targeted Initial Jupiter-Centered Keplerian Elements for Triple Flyby ...................... 19

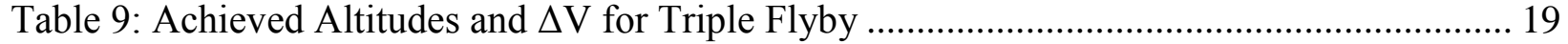

Table 10: Achieved Final Orbit Characteristics.................................................................... 20

Table 11: BPM Parameters ...................................................................................................... 22

Table 12: Spacecraft State Just After Earth Escape Maneuver................................................. 22

Table 13: Achieved Jupiter-Centered Keplerian Elements at First Apojove............................. 23

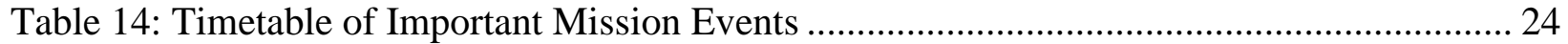

Table 15: Spacecraft Initial State, Jupiter-Centered Ecliptic J2000 ....................................... 28

Table 16: Standard Deviations for State Errors ................................................................... 32

Table 17: Cassini 2007-02 Maneuver Execution Error Model [39] ........................................ 35

Table 18: Expected Nominal Parameters from GMAT Model ............................................. 38

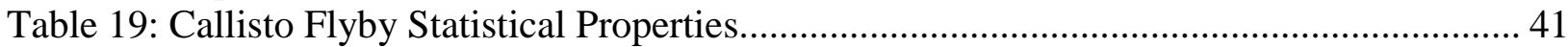

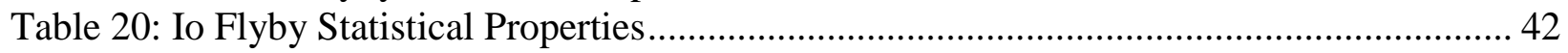

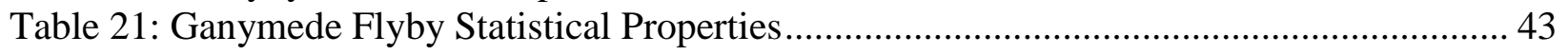

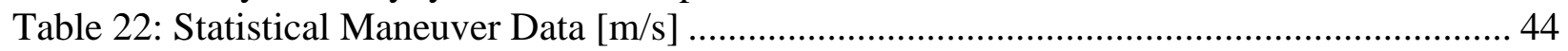

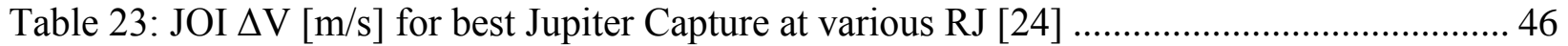

Table 24: Given Physical Constants ................................................................................ 52

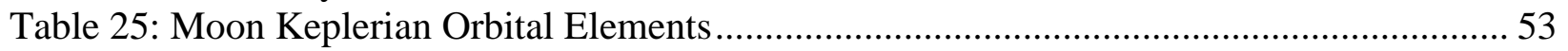

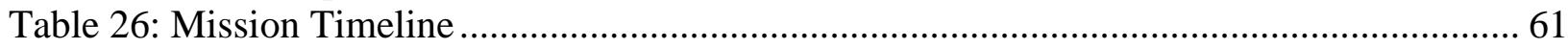

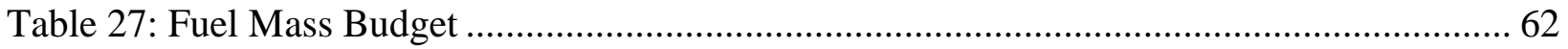




\section{Nomenclature}

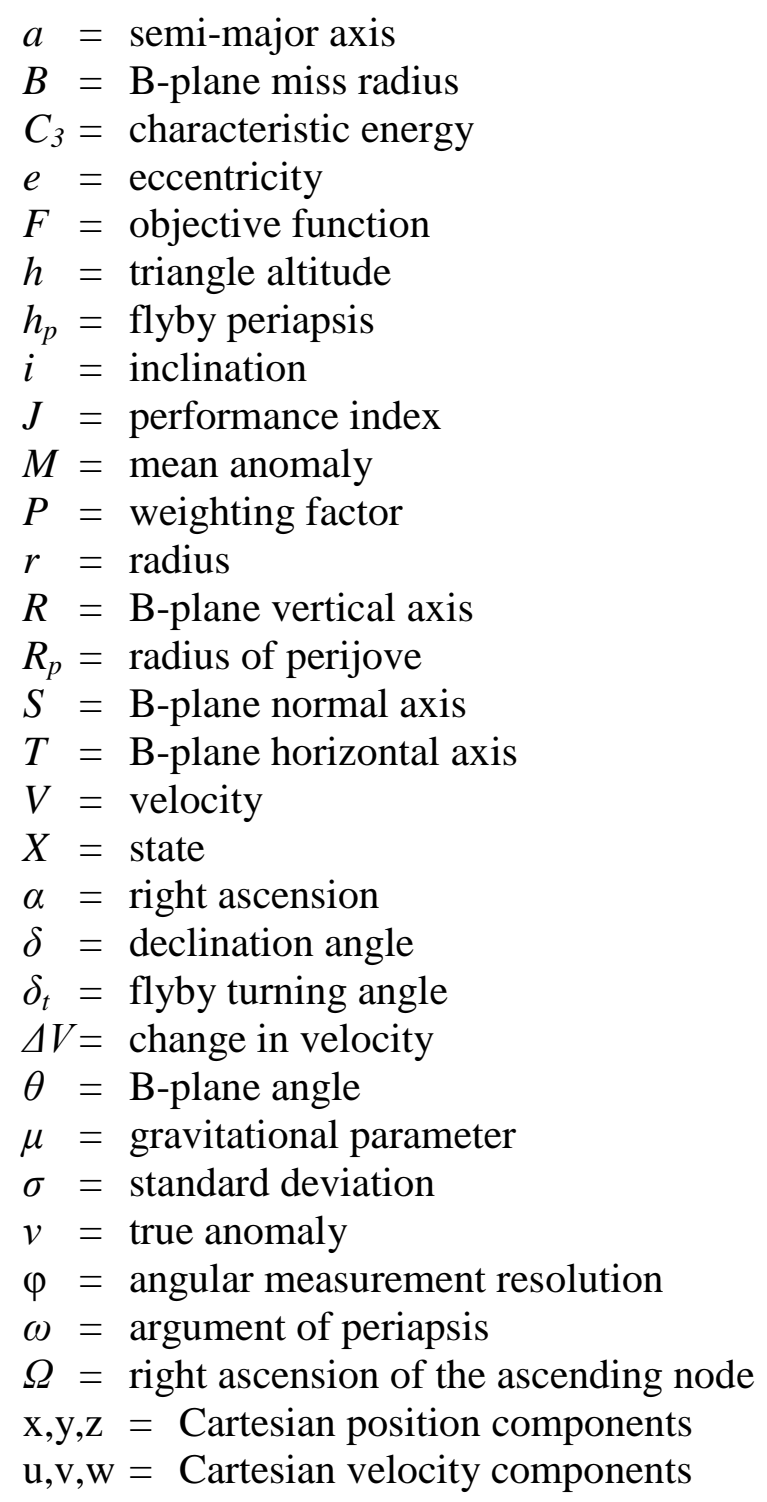




\title{
Mission Design, Guidance, and Navigation of a Callisto-Io-Ganymede Triple Flyby Jovian Capture
}

\author{
Alan M. Didion ${ }^{\mathrm{i}}$ \\ West Virginia University, Morgantown, West Virginia 26506
}

\section{Introduction}

$\mathrm{G}$ RAVITY-assist trajectories have been used in a number of deep space missions. The Galileo and Cassini missions both used several gravity-assist flybys of planets to obtain enough orbital energy to reach their destinations (Jupiter and Saturn, respectively) [1-8]. As Galileo arrived at Jupiter, it captured into orbit about Jupiter using a gravity assist of Io and a sizable impulsive $\Delta \mathrm{V}$ from its main engine. The Io gravity-assist reduced the $\Delta \mathrm{V}$ required to capture into Jupiter orbit by $185 \mathrm{~m} / \mathrm{s}$ [3]. After Galileo captured into Jupiter orbit, it performed dozens of gravity-assist flybys of Jupiter's Galilean moons, which have provided much scientific knowledge about the gravity fields and geology of each moon [9-12].

The use of gravity assists of planetary moons to reduce the $\Delta \mathrm{V}$ required to capture into planetary orbit is called "satellite-aided capture". While most papers discussing satellite-aided capture have focused on using only one satellite gravity assist to aid in the capture [13-18], several have proposed using two [19-23] or three [21-26] of Jupiter's Galilean moons to capture a spacecraft into orbit about Jupiter. Using three gravity-assists of Jupiter's Galilean moons is called "triple-satellite-aided capture". Further work by Lynam demonstrated that rare, impractical quadruple-satellite-aided capture opportunities do exist, but occur only once a decade and typically suffer from deep incursions into Jupiter's harsh radiation environment [27].

\footnotetext{
${ }^{i}$ M.S. Candidate, Department of Mechanical \& Aerospace Engineering, ESB, Evansdale Dr., Student Member AIAA
} 
Since Jupiter has four massive Galilean moons and any three of these moons can be encountered in differing orders, there are numerous permutations for triple-satellite-aided capture. Lynam et al. [21-22] discovered four geometrically possible "Laplacian triple-satelliteaided capture" sequences that use gravity assists of each of the Galilean moons that are involved in the 4:2:1 Laplace resonance: Ganymede, Europa, and Io. While these sequences occur more frequently than other triple-satellite-aided captures, they have a few deficiencies that make them less useful than more optimal double- and triple-satellite-aided capture sequences. These deficiencies include having low perijoves (two have perijoves $\left[\mathrm{R}_{\mathrm{p}}\right.$ 's] of slightly higher than 1 Jupiter radii [RJ]; two have $\mathrm{R}_{\mathrm{p}}$ 's of $2 \mathrm{RJ}$ ) that would increase radiation exposure and have lower $\Delta \mathrm{V}$ savings for most mission scenarios.

In addition to Laplacian triple-satellite-aided capture, the other three combinations of satellites are Callisto-Ganymede-Io, Callisto-Ganymede-Europa, and Callisto-Europa-Io triple flybys. Of these combinations, Callisto-Europa-Io is the definitive worst since Callisto and Europa are the weakest satellites for gravity-assist capture and must have a perijove that is below $6 \mathrm{RJ}$ to encounter Io, which is deep within Jupiter's radiation environment. Callisto-GanymedeEuropa sequences show some promise since they can have higher perijoves (Europa has an orbit of about 9.4 RJ), however they still suffer from the weaknesses of Callisto and Europa as gravity-assist capture bodies. The most promising combination is Callisto-Ganymede-Io since Ganymede and Io are the strongest of the Galilean moons for gravity-assist capture, although they also suffer from low perijoves of less than 6 RJ.

Although Callisto, Ganymede, and Io are the best combination of three moons to use for triple-satellite-aided capture, since they can be encountered in different orders, there are actually eight geometrically possible permutations. Lynam [25-26] developed a method that was able to 
find promising trajectories for two of these eight permutations: Callisto-Ganymede-Io-perijove and Callisto-Ganymede-perijove-Io.

This thesis is structured as follows. In Chapter 1, a suitable analysis candidate for the triplesatellite-aided capture sequence described was chosen by filtering existing broad search data. The chosen candidate was then verified through computer simulation and ultimately optimized for minimal fuel consumption. Additionally, the trajectory was back-propagated through interplanetary space to a feasible Earth-escape trajectory. In Chapter 2, this candidate was further refined in an entirely new dynamical model written by the author and subjected to realistic perturbations and knowledge uncertainties based on ephemeris, navigation, and maneuver execution inaccuracies. Chapter 3 stands alone, describing the author's contribution to his group's solution for the $8^{\text {th }}$ annual Global Trajectory Optimisation Competition (GTOC8). 


\section{Chapter 1: Design of an Impulsive Trajectory from Earth to Triple Flyby Jovian Capture}

This chapter builds on the work of Lynam by investigating one of the other six triple-satelliteaided capture trajectory permutations: the Callisto-Io-perijove-Ganymede sequence, wherein the spacecraft performs a double-flyby (Callisto, Io) and then performs an impulsive chemical maneuver at perijove before an outbound Ganymede flyby to close the orbit. It refines the heuristic reduction methods that Lynam used to find triple-satellite-aided capture sequences and it concentrates on finding ballistic trajectories from Earth to Jupiter that would use chemical propulsion rather than the low-thrust trajectories that Lynam [25-26] used. The trajectory candidate herein features the use of an interplanetary broken-plane maneuver (BPM) to ensure that the spacecraft's original trajectory plane intersects with an Earth encounter and that its final trajectory plane is coplanar with the orbits of the Galilean moons. Below, Figure 1 shows a basic diagram of how a triple-satellite-aided capture trajectory would look. Note how the final trajectory uses a triple flyby to close the orbit, as opposed to the hyperbolic incoming trajectory. Also note that the Ganymede flyby, being after perijove, must occur on the "left" side of the moon from the perspective of the spacecraft, i.e., this flyby features a negative "Bdot $T$ " value, as will be shown in the following sections. 


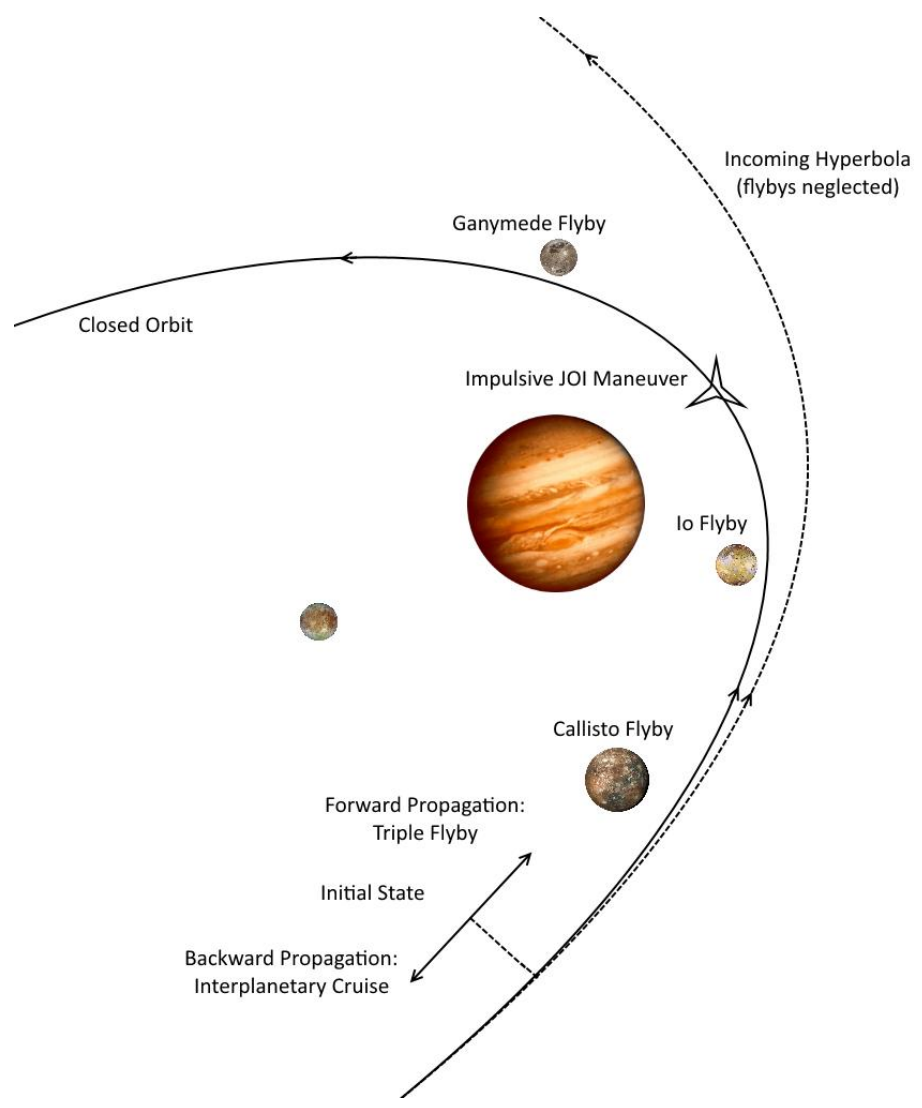

Figure 1: Basic Diagram of a Triple-Satellite-Aided Capture

\section{A. Flyby Candidate Filtering \& Selection}

The first priority in this analysis was to select a suitable candidate from given broad-search data and determine what qualities should be found in a "desirable candidate". This section describes the process of identifying desirable qualities in a candidate and filtering the data accordingly. Furthermore, desirable qualities for a fully targeted trajectory are identified and tabulated. These values will be used later as a general metric of success when examining the results of the optimization process.

\section{Candidate Selection Process}

To begin, a suitable candidate was chosen from a collection of approximately twenty thousand theoretically feasible capture orbits between the year 2024 and 2034 by sorting according to desirable properties. (This collection of trajectories was generated using a similar 
method to that of Lynam [25-26].) Candidate selection was performed by first choosing to use an incoming, rather than outgoing, Earth flyby trajectory and then eliminating all trajectories without an equatorial Callisto flyby; this reduced the number of candidates to approximately three hundred. All trajectories with incoming $\mathrm{R}_{\mathrm{p}}$ 's of less than $3 \mathrm{RJ}$ were then eliminated, as lower periapsides would expose the spacecraft to unnecessarily large amounts of radiation from Jupiter. Finally, the remaining trajectories were sorted from least to most required interplanetary $\Delta \mathrm{V}$. These criteria however are not able to account for the $\Delta \mathrm{V}$ required during the actual triplesatellite-aided capture, and could not be expected to unless each trajectory candidate was analyzed individually, which is not within the scope of this analysis. Instead, the chosen candidate will be optimized as much as possible.

\section{Candidate Description}

The chosen candidate's initial parameters are detailed in Table 1 and Table 2 in Cartesian and Keplerian elements respectively. Note that the epochs are given in Gregorian Coordinated Universal Time (UTCG) and Modified Julian Date (MJD) respectively. The UTCG epoch is more intuitive, but MJD will be used directly in the software, which uses a non-standard reference epoch. These conditions represent the untargeted initial guess for the candidate. Later, in targeting an optimal trajectory, some of these values will change; these final values will be referred to as the targeted initial state. This state will be the point from which the triple-satelliteaided capture trajectory is propagated forward and the interplanetary transfer trajectory is propagated backward; thus, it lies at a point sufficiently far from Jupiter but in the neighborhood. It can be thought of as the point at which the spacecraft enters the sphere of influence of Jupiter, and the models used will also change upon crossing this point to reflect this. 
Table 1: Initial Guess for Jupiter-Centered Cartesian Elements

\begin{tabular}{lcrr}
\hline Parameter & Symbol & Value & Units \\
\hline \hline Epoch & {$[\mathrm{N} / \mathrm{A}]$} & 2025 FEB 03 02:01:24 & UTC G \\
X Position & $\mathrm{X}$ & -4589880.285 & {$[\mathrm{~km}]$} \\
Y Position & $\mathrm{Y}$ & -8789.404 & {$[\mathrm{~km}]$} \\
Z Position & $\mathrm{Z}$ & -61966.500 & {$[\mathrm{~km}]$} \\
X Velocity & $\mathrm{Vx}$ & 9.240 & {$[\mathrm{~km} / \mathrm{s}]$} \\
Y Velocity & $\mathrm{Vy}$ & -1.843 & {$[\mathrm{~km} / \mathrm{s}]$} \\
Z Velocity & $\mathrm{Vz}$ & 0.067 & {$[\mathrm{~km} / \mathrm{s}]$} \\
V infinity & $\mathrm{V}_{\infty}$ & 5.622 & {$[\mathrm{~km} / \mathrm{s}]$} \\
\hline
\end{tabular}

Table 2: Initial Guess for Jupiter-Centered Keplerian Elements

\begin{tabular}{lcrr}
\hline Parameter & Symbol & Value & Units \\
\hline \hline Epoch & {$[\mathrm{N} / \mathrm{A}]$} & 30709.584 & $\mathrm{MJD}$ \\
Semi-major Axis & $\mathrm{a}$ & -3774011.246 & {$[\mathrm{~km}]$} \\
Eccentricity & $\mathrm{e}$ & 1.07364 & {$[\mathrm{~N} / \mathrm{A}]$} \\
Inclination & $\mathrm{i}$ & 1.950 & {$[\mathrm{deg}]$} \\
RAAN & $\Omega$ & 336.747 & {$[\mathrm{deg}]$} \\
AOP & $\omega$ & 347.910 & {$[\mathrm{deg}]$} \\
True Anomaly & $v$ & 215.465 & {$[\mathrm{deg}]$} \\
Hyperbolic Perijove & $\mathrm{R}_{\mathrm{p}}$ & 3.887 & {$[\mathrm{RJ}]$} \\
V infinity & $\mathrm{V}_{\infty}$ & 5.622 & {$[\mathrm{~km} / \mathrm{s}]$} \\
\hline
\end{tabular}

It will be shown that of particular impact on the trajectory's "desirable qualities" are the starting epoch and eccentricity, and inclination to a lesser degree. Some parameters will be shown to correlate heavily with changes in particular legs of the trajectory while others will have a more subtle influence.

\section{Goal Definitions}

Prior to any targeting, the GMAT default script was modified as will be discussed in the next section, adding the Galilean moons into the simulation environment and creating the necessary coordinate systems to accurately display the solution. An initial propagation of the approximation candidate yielded a rough triple flyby trajectory through the Jupiter system which did not achieve the stated goals, so targeting had to be performed in order to refine the trajectory to a usable state. To define "usable state", some reasonable but adjustable parameters were set as targets for the triple flyby trajectory. These target values are detailed in Table 3 below, and 
represent the features of a feasible, efficient capture sequence to place a spacecraft into a desirable two-hundred day equatorial science orbit around Jupiter. It is important to note here: recall that the sequence includes a ballistic double flyby, followed by a Jupiter Orbit Insertion (JOI) burn at perijove which then allows the spacecraft to ballistically fly by Ganymede for the exact assist necessary to place it in a relatively equatorial, closed, two-hundred day Jupiter orbit. Table 4 also details the parameters that will be referred to as describing a "desirable", marketable final orbit. These parameters ensure that the final science orbit is useful and that the incoming trajectory does not expose the spacecraft to excessive amounts of Jupiter's radiation.

Table 3: Altitude and $\Delta V$ Targets for a Desirable Trajectory

\begin{tabular}{lrr}
\hline Parameter & Value & Units \\
\hline \hline Callisto Altitude & 100.0 & {$[\mathrm{~km}]$} \\
Callisto $\theta$ & 0.0 & {$[\mathrm{deg}]$} \\
Io Altitude & 300.0 & {$[\mathrm{~km}]$} \\
Io $\theta$ & 0.0 & {$[\mathrm{deg}]$} \\
JOI Burn Magnitude & 0.230 & {$[\mathrm{~km} / \mathrm{s}]$} \\
Ganymede Altitude & 100.0 & {$[\mathrm{~km}]$} \\
Ganymede $\theta$ & 180.0 & {$[\mathrm{deg}]$} \\
\hline
\end{tabular}

Table 4: Limits and Recommendations for a Desirable Trajectory

\begin{tabular}{lrr}
\hline Parameter & Value & Units \\
\hline \hline Orbital Period (final) & $\sim 200$ & {$[$ days] } \\
Inclination (final) & $\sim 0$ & [deg] \\
Perijove (burn) & $>3.0$ & {$[\mathrm{RJ}]$} \\
\hline
\end{tabular}

The flyby altitudes will be fairly low to make the most use of the gravity assists as well as to gather science data during close approaches of the moons, especially while passing through the volcanic plumes of Io, which can exceed the $300 \mathrm{~km}$ targeted periapsis of this mission. The "BdotR" values at Callisto and Ganymede are both targeted to be approximately zero; this will help ensure that the interplanetary and final trajectories, respectively, are approximately equatorial with respect to their central body. The Io flyby will use an off-plane BdotR value to 
reconcile the resulting inclination differences between the two extremes, using Io's large gravity assist potential in place of the equivalent large $\Delta \mathrm{V}$ that would be required. The flyby altitudes will mainly dictate appropriate "BdotT" values, which are the most influential geometric factor in acquiring equivalent $\Delta \mathrm{V}$ from gravity assist maneuvers.

\section{B. Trajectory Design \& Optimization in GMAT}

In order to generate high-fidelity orbit simulations and formulate optimal conditions for a triple-satellite-aided capture maneuver, the NASA General Mission Analysis Tool (hereafter referred to as GMAT) software was used. The GMAT software was chosen due to its adaptability to such esoteric mission profiles such as that described in this thesis as well as its open source availability and computational flexibility and accuracy. While less widely used than other similar astrodynamic propagation software like Systems Tool Kit (STK), it is easily reconfigured to fit mission specifics (such as addition of the Galilean moons), provides a simple GUI and script editing interface, and is capable of interfacing with MATLAB in order to use its optimization subroutines, in addition to being freely available in its full-release form online. This section describes the setup of this software and special adjustments made in order to properly simulate this complex situation. Furthermore, some prerequisite material such as coordinate frames will be defined. And finally, the methodology behind building the propagation and optimization routines will be explained in detail.

\section{Software Configuration and Setup}

The GMAT software provides a default environment which includes the sun, each of the eight planets, Pluto, and the earth's moon. Additionally, the default scenario includes multiple Earthcentered coordinate systems and an Earth-orbiting generic spacecraft. For the purposes of a Jovian triple-satellite-aided capture trajectory, the default spacecraft can be disposed of in favor 
of a customized spacecraft while the Earth-centered coordinate systems can be ignored, but not deleted. In order to model the Jovian system and its Galilean moons, additional celestial bodies were added with the gravitational parameter, equatorial radius, texture map, and SPICE ephemeris data corresponding to each of the four major moons. All interplanetary propagation will incorporate gravitational effects of point masses representing the sun, all of the planets, and Pluto, while all propagation within the Jovian sphere of influence will incorporate such effects from the sun, Jupiter, and the four Galilean moons. To properly display each of the three flyby maneuvers and provide relevant orbital parameters, an "orbit view" graphical output window was created corresponding to each of the three new moon coordinate systems as well as a Jupiter-centered and Sun-centered coordinate systems. Because the Galilean moons must be added manually into the simulation environment, their precise radii and gravitational parameters were required. These parameters are set according to the values provided in the JPL Solar System Dynamics planetary satellite physical parameters table, and are detailed below in Table 5 $[28-31]{ }^{\mathrm{ii}}$

Table 5: Physical Parameters of the Galilean Moons

\begin{tabular}{lcc}
\hline \multicolumn{1}{c}{ Body } & $\begin{array}{c}\boldsymbol{\mu} \\
{\left[\mathrm{km}^{3} / \mathrm{s}^{2}\right]}\end{array}$ & $\begin{array}{c}\text { Mean Radius } \\
{[\mathrm{km}]}\end{array}$ \\
\hline \hline Io & 5959.916 & 1821.6 \\
Europa & 3202.739 & 1560.8 \\
Ganymede & 9887.834 & 2631.2 \\
Callisto & 7179.289 & 2410.3 \\
\hline
\end{tabular}

To propagate astrodynamical systems, GMAT employs a numerical integrator which can be adjusted with custom integrator types, step sizes, and stopping tolerances (acceptable accuracy), among other parameters. As is common practice with close flyby maneuvers, the default

\footnotetext{
${ }^{i i}$ Planetary Satellite Physical Parameters, JPL Solar System Dynamics, [online], http://ssd.jpl.nasa.gov/?sat_phys_par (Accessed: 6 January 2014)
} 
"RungeKutta89" integrator was changed to the "PrinceDormand78" integrator, which is specifically designed for the required type of adaptive step sizing, yielding higher accuracy as the spacecraft approaches the stronger gravitational field close to celestial bodies and higher computational speed in interplanetary voids where large steps can be taken. Using these specially tailored tools, GMAT was used to refine initial guess parameter approximations into a complete orbital profile with precise $\Delta \mathrm{V}$ and moon body-plane (B-plane) targets based on numerical calculation and ephemeris data. It is important to note that with integration techniques that use adjustable step sizing, such as PrinceDormand78, safeguards must be inserted into the script to stop propagation if the solution breaches the surface of a celestial body, which is treated as a point mass. If this is not done and the solution approaches the core of a body, its gravitational singularity, the adjustable step size will shrink exponentially and severely hinder the solving

process. In this situation, such safeguards were accomplished by causing the solver to revert to the "JupiterOnly" propagator if the spacecraft was below the surface of a moon. This propagator neglected the gravitational effects of the moons and the spacecraft would drift through the moon to be reset and that run deleted without slowing down the process.

\section{Definition of Flyby Coordinate System}

Moon flyby maneuvers will be described throughout this analysis using the flyby body plane (B-plane) parameters. The parameters of most importance are the "BdotT" and "BdotR" dot products (as denoted in GMAT), shown in Figure 2 below, which quantify respectively the horizontal and vertical components of the "B" miss radius of the incoming hyperbola. These quantities, it will be shown, can be correlated to the change in the spacecraft's Jupiter-centered orbital energy and inclination, respectively. This means that flybys with lower BdotT values will serve to drastically change the orbital energy of the spacecraft's Jupiter-centered orbit while, 
similarly, high BdotR flybys will drastically change the inclination of the orbit. In this figure, the "S" vector is simply the unit vector centered on the moon parallel to the incoming velocity asymptote, from which "R" and " $T$ " are orthogonally derived. These vectors, with the miss radius, $B$, and $B$-plane angle, $\Theta$, fully describe a celestial flyby.

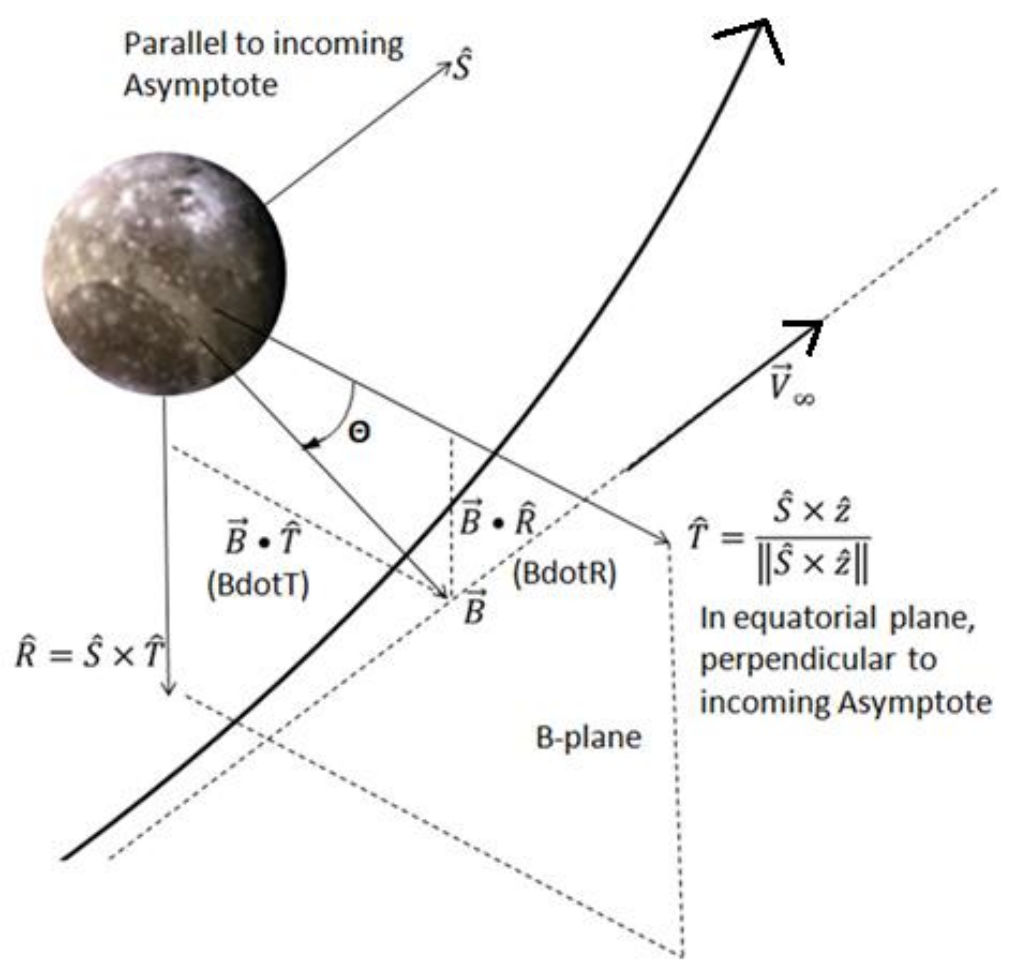

Figure 2: Definition of the B-plane Coordinate System

\section{Script Architecture}

To attempt to meet these conditions, initial rough targeting scripts were formulated that aimed to determine an initial state that would ballistically complete a suitable flyby of Callisto and Io without additional $\Delta \mathrm{V}$ adjustment. In order to achieve this, it was necessary to identify all variables available and examine those whose adjustment would serve to achieve different target parameters. By examining the desired mission profile, it became clear that these variables were the six Keplerian elements and starting epoch of the flyby trajectory as it enters Jupiter's sphere of influence (SOI), the three flyby altitudes, the JOI burn $\Delta \mathrm{V}$ magnitude, and the Io B-plane 
angle. The Jupiter SOI elements were to then be ballistically back-propagated to a broken-plane maneuver to flyby Earth. This yielded variables in the interplanetary scenario of time of flight (TOF) from Jupiter SOI to the BPM and the three BPM burn Cartesian elements. The BPM burn elements and TOF are of importance primarily to the interplanetary cruise scenario. Furthermore, the Jupiter SOI-state (or incoming) semi-major axis will greatly influence the interplanetary trajectory, but the flyby sequence will be hardly affected by its alteration. Likewise, the Callisto flyby will be strongly governed by the incoming state's epoch, inclination, right-ascension of the ascending node, and argument of periapsis; the subsequent Callisto flyby altitude will dictate the Io flyby parameters. The subsequent Io flyby altitude and B-plane angle will dictate the Ganymede flyby parameters, fully ballistic with the exception of a pure retrograde JOI burn at perijove. All of these parameters, with Ganymede's flyby included, will then determine the final orbit's elements and the most important parameters: the orbital period, $\mathrm{R}_{\mathrm{p}}$, and inclination.

Because of the somewhat disjoint nature of the effects of the variables in the problem, a "nested loop" structure was used to target individual phases of the trajectory, organized as follows. The interplanetary and triple flyby trajectories are mostly disjoint, patched only by the Keplerian elements at the exact epoch of "initial state" on the Jupiter approach hyperbola as defined by the initial guesses. Therefore, to simplify the programming approach, the triple flyby was modeled in a forward propagated script while the interplanetary trajectory was modeled in a completely separate, backwards propagated script sharing the spacecraft "initial state". The triple flyby trajectory is split into a nested double loop structure, with an internal differential corrector loop varying the incoming epoch, inclination, right ascension of the ascending node, and argument of periapsis. This inner loop was set to achieve given targets for Callisto and Io flyby BdotT and BdotR values. The outer differential corrector loop varied incoming eccentricity and 
the Io B-plane angle. Each outer loop iteration also ran the inner loop until convergence and then propagated the results to perijove where it executed the chosen $\Delta \mathrm{V}$ and continued propagating to Ganymede. There, it attempted to achieve a proper Ganymede flyby altitude to achieve the desired final orbital period. It is important to note that in this structure, each iteration of the outer loop required a complete convergence cycle of the inner loop, and so the outer loop can require considerable time and computational power to converge. The script logic for the triple flyby solver is shown the form of a flow chart in Figure 3.

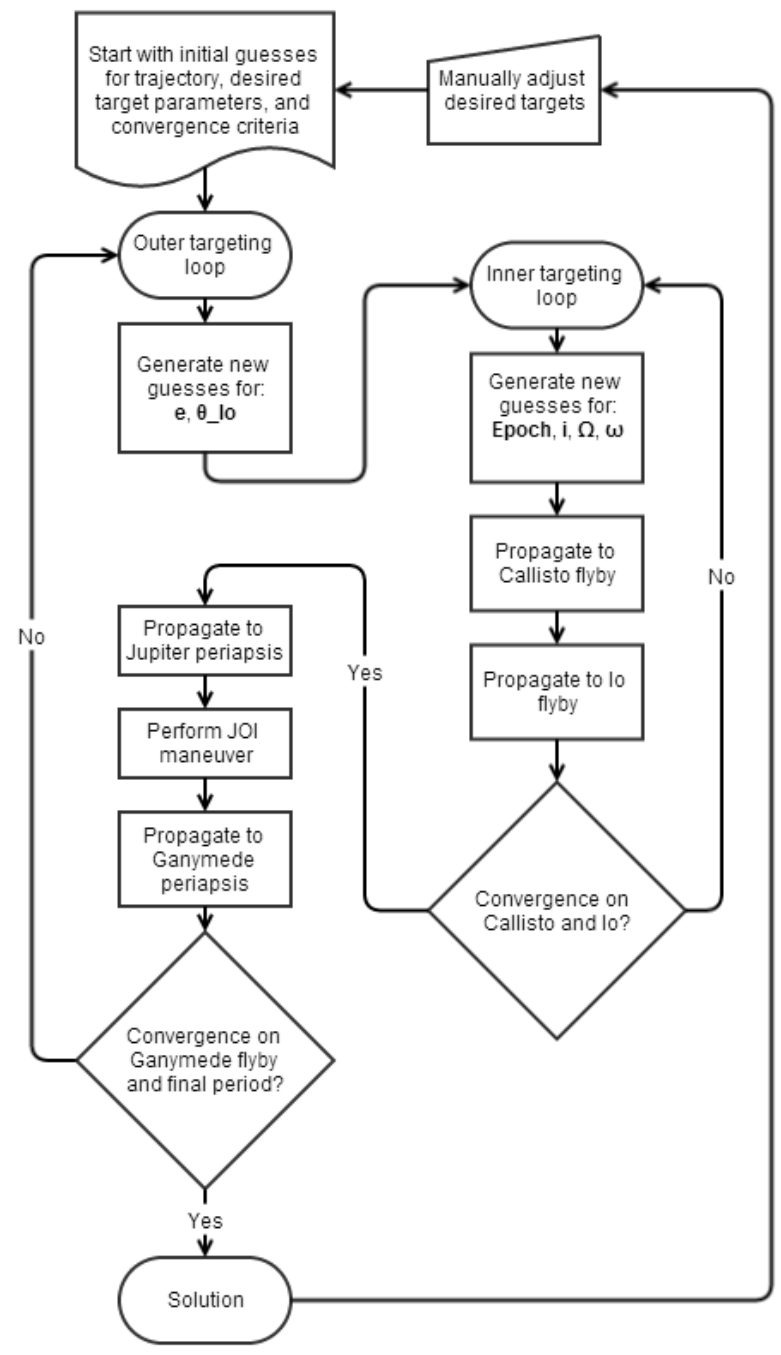

Figure 3: Triple Flyby "Nested Solver" Flow Chart 


\section{Optimizer Architecture}

By using a MATLAB script interface, GMAT can solve problems with non-linear constraints through an optimization routine included in the MATLAB optimization toolbox, known by the handle "FminconOptimizer". This optimization routine allows GMAT to solve for the orbital parameters and flyby altitudes that allow a triple flyby situation requiring the minimum JOI maneuver $\Delta \mathrm{V}$ possible without being given a specific desired $\Delta \mathrm{V}$ value. This function was used also to minimize the deviation from a desired "reasonable" Earth periapsis radius corresponding to an Earth escape when targeting Earth during the interplanetary back-propagation phase. This was done by varying the semi-major axis of the Jupiter-centered hyperbola until the achieved Earth periapsis was "close" to one set by the investigator. Here, "reasonable" refers to a trajectory achievable by whatever launch vehicles are available for the mission and is widely variable. The details of the launch and escape phase are largely unexamined here as it lies beyond the scope of the investigation; as such, the tolerances on the interplanetary optimizer are large. However, the capabilities of the upcoming Space Launch System (SLS) and how it could be used for such an escape are detailed in a sub-section to follow. The addition of the optimization process helps ensure that no solution is selected with largely arbitrarily chosen constraints, but that every value within the simulation has been carefully optimized and shown to provide the best possible trajectory in terms of minimal propellant usage and adequate flyby altitude. The optimizer script logic for the triple flyby and interplanetary trajectories can be seen in Figure 4 and Figure 5 respectively. 


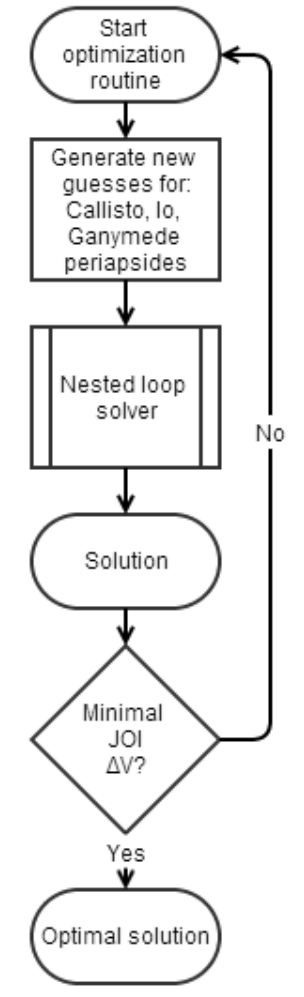

Figure 4: Triple Flyby Trajectory Optimization Flow Chart

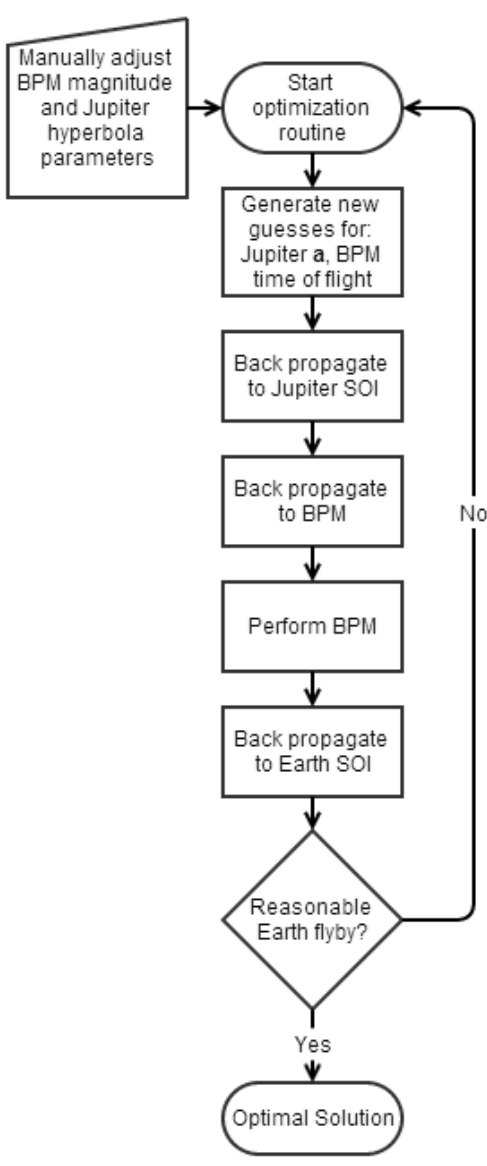

Figure 5: Interplanetary Trajectory Optimization Flow Chart

The flow of the interplanetary trajectory's script, as shown in Figure 5 consists of only one loop which varies the time of flight to BPM, and Jupiter centered semi-major axis at the initial state. It then propagated backwards to and executed the BPM before propagating further back to achieve an Earth encounter. Backwards propagation is identical to the previously used forward propagation, but with negative time-steps. This "split-propagation" scheme, with two scripts propagating in opposite directions, allows the two scripts to achieve the two halves of the mission independently and meet at a common point in space and time. Such an approach was necessary given the complexity of the triple-satellite-aided capture trajectory and its incredible sensitivity to initial conditions in contrast to the interplanetary trajectory's insensitivity to same. 
Furthermore, because the interplanetary trajectory exists over such large time and space constraints, it is easier for it to target a large range of patch conditions, as required to achieve the triple-satellite-aided capture.

\section{Launch Vehicle Considerations}

The most reasonable option for launch and interplanetary injection for such a mission as herein described would be through the use of the upcoming NASA Space Launch System (SLS) launch vehicle. Currently undergoing design reviews, the SLS will be a versatile new heavy-lift launch vehicle operated by the United States for the future of beyond-Earth exploration of all varieties. Table 6 details the projected performance for the two main SLS configurations.

Table 6: SLS Payload Injection Capabilities [32]

\begin{tabular}{lccc}
\hline \multicolumn{1}{c}{ Trajectory } & $\begin{array}{c}\mathbf{C}_{\mathbf{3}} \\
{\left[\mathbf{k m}^{2} / \mathbf{s}^{\mathbf{2}}\right]}\end{array}$ & $\begin{array}{c}\text { SLS Block 1 } \\
{[\mathbf{m t}]}\end{array}$ & $\begin{array}{c}\text { SLS Block 1b } \\
{[\mathbf{m t}]}\end{array}$ \\
\hline \hline Geostationary Orbit & & 14.60 & 26.80 \\
Trans-Mars Injection & 11.0 & 19.50 & 33.00 \\
2022 Europa EGA & 28.9 & 12.90 & 25.10 \\
2022 Europa Direct & 85.4 & 4.38 & 8.92 \\
Jupiter Trojan Belt & 90.0 & 3.96 & 7.59 \\
67P/C-G Comet Sample Return & 94.3 & 3.61 & 6.59 \\
Saturn/Titan/Enceladus & 106.0 & 2.72 & 5.12 \\
Uranus & 135.5 & 1.01 & 1.48 \\
\hline
\end{tabular}

Current estimates boast that the SLS Block 1 will be able to boost approximately 4.4 metric tons into a direct trajectory for Europa interception and Block 1b will be able to similarly inject $8.9 \mathrm{mt}$ [32]. For reference, Cassini-Huygens weighed approximately $5.7 \mathrm{mt}$ (wet, and with Huygens; $2.1 \mathrm{mt}$ dry). Below, find a figure of injected payload vs. $\mathrm{C}_{3}$ characteristic energy for both block configurations, compared against the United Launch Alliance (ULA) Delta IV Heavy, the current highest-capacity launch vehicle in operation. Not included is the SpaceX Falcon Heavy, which would perform between the Delta IV Heavy and the SLS. Note: one can expect 
that the direct interplanetary trajectory used here will be comparable to the "Europa Direct" data point.

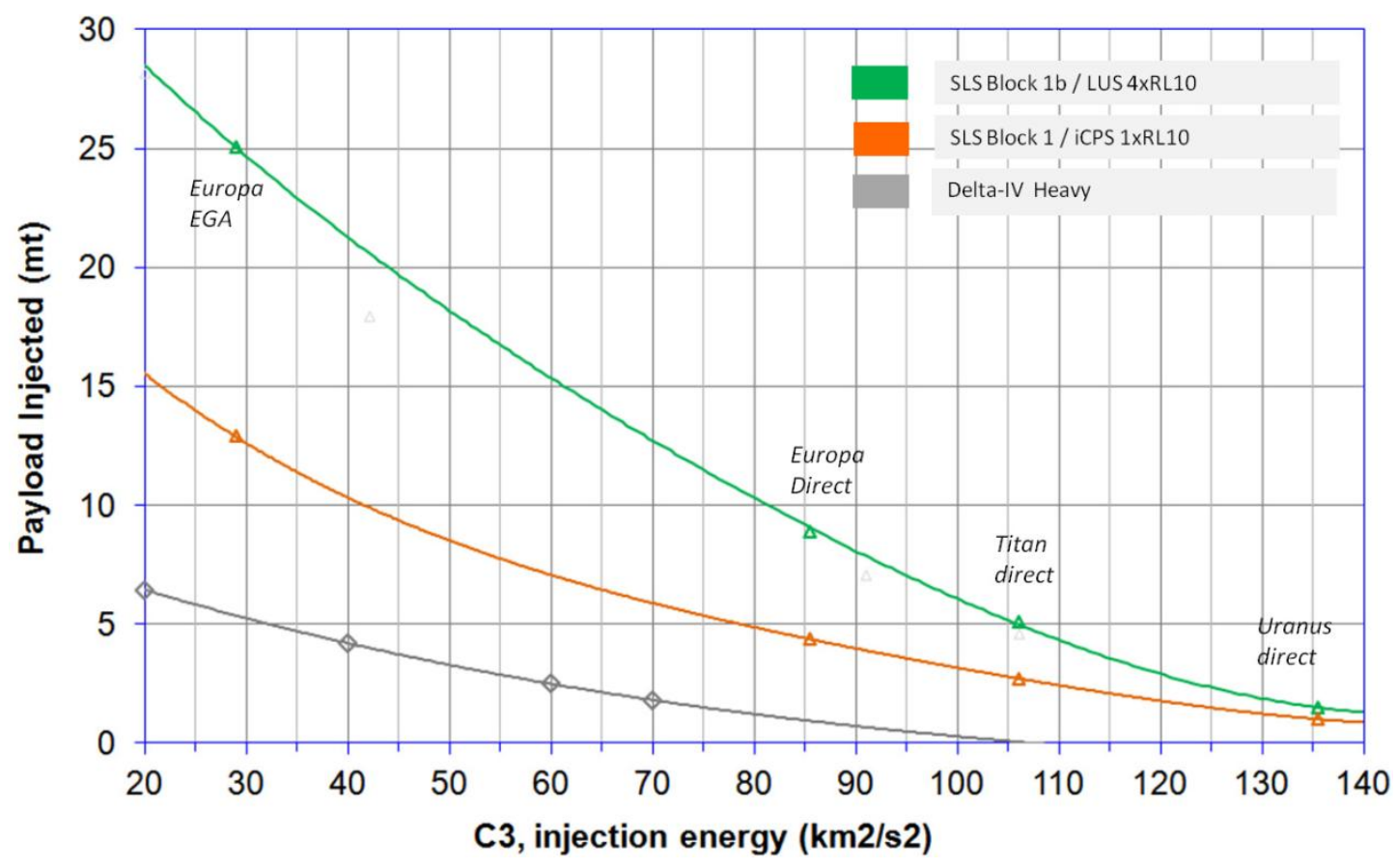

Figure 6: SLS Payload vs. $\mathrm{C}_{3}$ Capabilities [32].

\section{Results of GMAT Trajectory Optimization}

With proper setup, the patched, nested-loop optimizer script architecture produced a full impulsive trajectory from Earth to a precise triple-satellite-aided Jovian capture using only a minimal retrograde perijove maneuver and a single interplanetary broken plane maneuver. The mission successfully escaped Earth, cruised to Jupiter and captured using less propellant than comparable missions and guarantees science opportunities in the form of close flybys to three of the four Galilean moons and Jupiter prior to completion of the first Jupiter orbit and a desirable final orbit for the extent of the mission. The analysis concluded with the spacecraft at first apojove. At which point the orbit can be further adjusted to raise perijove and target a specific science flyby. 
Achieved mission parameters are detailed below in Tables 5-8; these can be compared to the initial guesses and goals as previously detailed in Tables 1-4. It can be seen that some restrictions have been relaxed both manually and by the solver routine in order to achieve the ultimate final period goal. Most notable is the relaxation of the constraint on the Callisto flyby altitude, allowed to fluctuate low in order to converge on the necessary Io flyby to achieve the desired Ganymede parameters.

Table 7: Targeted Initial Jupiter-Centered Cartesian Elements for Triple Flyby

\begin{tabular}{lcrr}
\hline Parameter & Symbol & Value & Units \\
\hline \hline Epoch & {$[\mathrm{N} / \mathrm{A}]$} & 02 Feb 2025 21:19:19 & UTC G \\
X Position & $\mathrm{X}$ & -4714128.923 & {$[\mathrm{~km}]$} \\
Y Position & $\mathrm{Y}$ & 68943.330 & {$[\mathrm{~km}]$} \\
Z Position & $\mathrm{Z}$ & -60914.940 & {$[\mathrm{~km}]$} \\
X Velocity & $\mathrm{Vx}$ & 9.078 & {$[\mathrm{~km} / \mathrm{s}]$} \\
Y Velocity & $\mathrm{Vy}$ & -1.970 & {$[\mathrm{~km} / \mathrm{s}]$} \\
Z Velocity & $\mathrm{Vz}$ & 0.060 & {$[\mathrm{~km} / \mathrm{s}]$} \\
V infinity & $\mathrm{V}_{\infty}$ & 5.704 & {$[\mathrm{~km} / \mathrm{s}]$} \\
\hline
\end{tabular}

Table 8: Targeted Initial Jupiter-Centered Keplerian Elements for Triple Flyby

\begin{tabular}{lcrr}
\hline Parameter & Symbol & Value & Units \\
\hline \hline Epoch & {$[\mathrm{N} / \mathrm{A}]$} & 30709.388 & $\mathrm{MJD}$ \\
Semi-major Axis & $\mathrm{a}$ & -3894011.246 & {$[\mathrm{~km}]$} \\
Eccentricity & $\mathrm{e}$ & 1.07342 & {$[\mathrm{~N} / \mathrm{A}]$} \\
Inclination & $\mathrm{i}$ & 1.956 & {$[\mathrm{deg}]$} \\
RAAN & $\Omega$ & 336.929 & {$[\mathrm{deg}]$} \\
AOP & $\omega$ & 346.780 & {$[\mathrm{deg}]$} \\
True Anomaly & $v$ & 215.465 & {$[\mathrm{deg}]$} \\
Hyperbolic Perijove & $\mathrm{Rp}$ & 3.999 & {$[\mathrm{RJ}]$} \\
\hline
\end{tabular}

Table 9: Achieved Altitudes and $\Delta \mathrm{V}$ for Triple Flyby

\begin{tabular}{lrr}
\hline Parameter & Value & Units \\
\hline \hline Callisto Altitude & 54.8 & {$[\mathrm{~km}]$} \\
Callisto $\theta$ & 0.0 & {$[\mathrm{deg}]$} \\
Io Altitude & 279.0 & {$[\mathrm{~km}]$} \\
Io $\theta$ & -26.3 & {$[\mathrm{deg}]$} \\
JOI Burn Magnitude & 0.264 & {$[\mathrm{~km} / \mathrm{s}]$} \\
Ganymede Altitude & 97.3 & {$[\mathrm{~km}]$} \\
Ganymede $\theta$ & 168.6 & {$[\mathrm{deg}]$} \\
\hline
\end{tabular}


Table 10: Achieved Final Orbit Characteristics

\begin{tabular}{lrr}
\hline Parameter & Value & Units \\
\hline \hline Orbital Period (final) & 198.913 & [days] \\
Inclination (final) & 2.329 & [deg] \\
Perijove (JOI) & 3.237 & [RJ] \\
\hline
\end{tabular}

As shown in Table 9, the optimization sequence was unable to achieve both the desired period and the $\Delta \mathrm{V}$ recommendation within flyby altitude restrictions, and the choice was made to sacrifice more propellant in favor of the lower orbital period. However, the perijove at which the JOI maneuver is performed is above the desired minimum (of $3 \mathrm{RJ}$ ), safeguarding the spacecraft from unnecessary radiation exposure. The periapsis altitude of the final orbit, however, was low and would require adjustment for a small amount of prograde $\Delta \mathrm{V}$ at the moment of first apoapsis, which has not been modeled here. Such periapsis adjustment has been left to those tailoring the mission to their specific objectives. A graphic representation of the capture orbit through the Jovian system can be seen in Figure 7. In this figure, the incoming hyperbola intersects the orbit of each moon (except Europa) at the point of each respective flyby and the spacecraft is captured into a final closed orbit with the apojove out of frame. Each flyby is shown individually in Figure 8. 


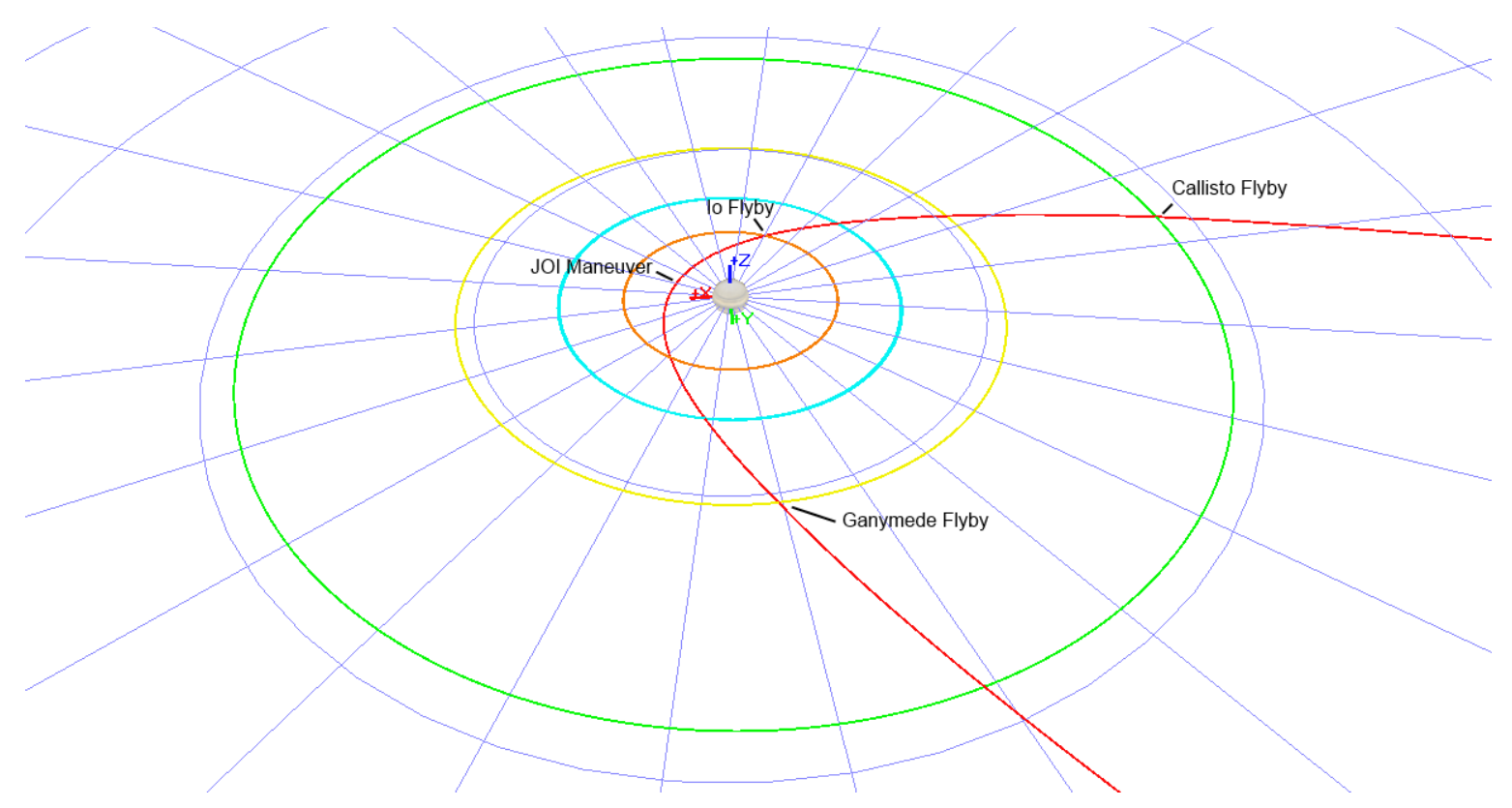

Figure 7: GMAT Triple Flyby Orbit Plot

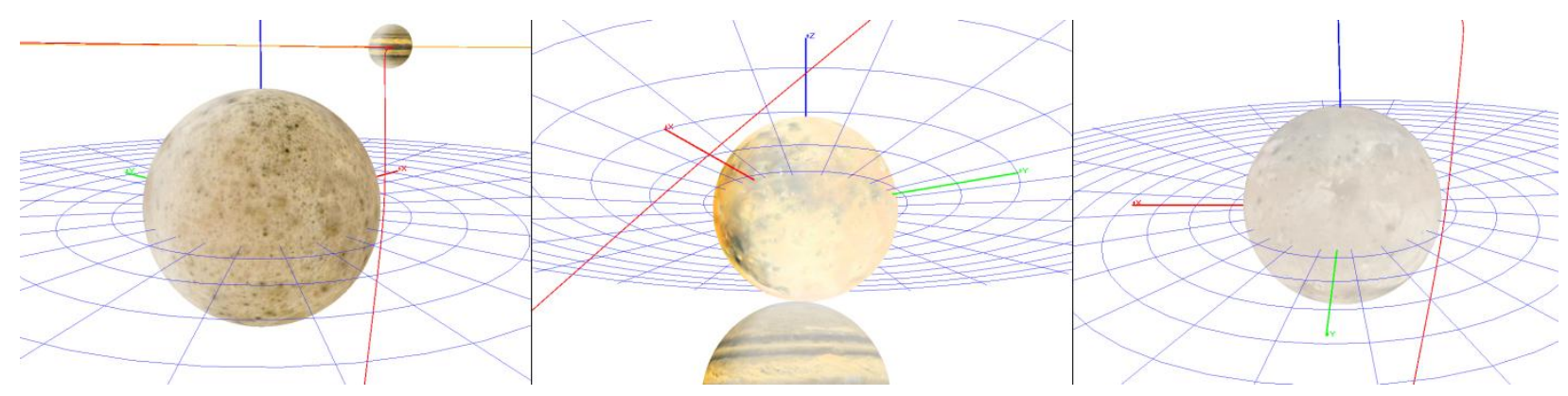

Figure 8: GMAT Plots for the Callisto, Io, and Ganymede Flybys Respectively

Back-propagating from the "targeted initial state" given in Tables 5-6, the interplanetary transfer trajectory was optimized to provide a suitable Earth escape. The Earth escape parameters were somewhat arbitrary and can be adjusted to suit the specific target mission or launch capabilities. For the purpose of initial rough targeting, an arbitrary Earth escape from a circular, 20,000 km orbit was used. The BPM specifics are detailed below in Table 11, as are the Earth escape specifics in Table 12. As shown, the BPM requires a total of approximately $12 \mathrm{~m} / \mathrm{s}$ of $\Delta \mathrm{V}$ to connect the Jupiter state with the Earth state given in Table 12. The resultant Earth $\mathrm{V}_{\infty}$ is approximately $9.253 \mathrm{~km} / \mathrm{s}$, corresponding to a $\mathrm{C}_{3}$ characteristic energy of $83.618 \mathrm{~km}^{2} / \mathrm{s}^{2}$. 
Recalling Table 6 and Figure 6, this $\mathrm{C}_{3}$ is comparable to that of the "2022 Europa Direct" trajectory at $85.4 \mathrm{~km}^{2} / \mathrm{s}^{2}$, meaning that the SLS Block 1 could inject a probe comparable to Cassini into the trajectory described herein. Further, Block $1 \mathrm{~b}$ could significantly increase this injected mass. The interplanetary transfer, Earth and Jupiter intercepts, and BPM location are shown in a heliocentric view in Figure 9.

Table 11: BPM Parameters

\begin{tabular}{lrr}
\hline Parameter & Value & Units \\
\hline \hline TOF & 610.0 & {$[$ days] } \\
$\Delta \mathrm{V}_{\mathrm{x}}$ & 7.50 & {$[\mathrm{~m} / \mathrm{s}]$} \\
$\Delta \mathrm{V}_{\mathrm{y}}$ & -8.36 & {$[\mathrm{~m} / \mathrm{s}]$} \\
$\Delta \mathrm{V}_{\mathrm{z}}$ & 3.50 & {$[\mathrm{~m} / \mathrm{s}]$} \\
$\Delta \mathrm{V}$ & 11.76 & {$[\mathrm{~m} / \mathrm{s}]$} \\
\hline
\end{tabular}

Table 12: Spacecraft State Just After Earth Escape Maneuver

\begin{tabular}{lcrr}
\hline Parameter & Symbol & Value & Units \\
\hline \hline Epoch & {$[\mathrm{N} / \mathrm{A}]$} & 29755.579 & $\mathrm{MJD}$ \\
Semi-major Axis & $\mathrm{a}$ & -4655.913 & {$[\mathrm{~km}]$} \\
Eccentricity & $\mathrm{e}$ & 5.31685 & {$[\mathrm{~N} / \mathrm{A}]$} \\
Inclination & $\mathrm{i}$ & 165.032 & {$[\mathrm{deg}]$} \\
RAAN & $\Omega$ & 273.631 & {$[\mathrm{deg}]$} \\
AOP & $\omega$ & 181.598 & {$[\mathrm{deg}]$} \\
True Anomaly & $v$ & 0.000 & {$[\mathrm{deg}]$} \\
Characteristic Energy & $\mathrm{C}_{3}$ & 83.618 & {$\left[\mathrm{~km}^{2} / \mathrm{s}^{2}\right]$} \\
\hline
\end{tabular}




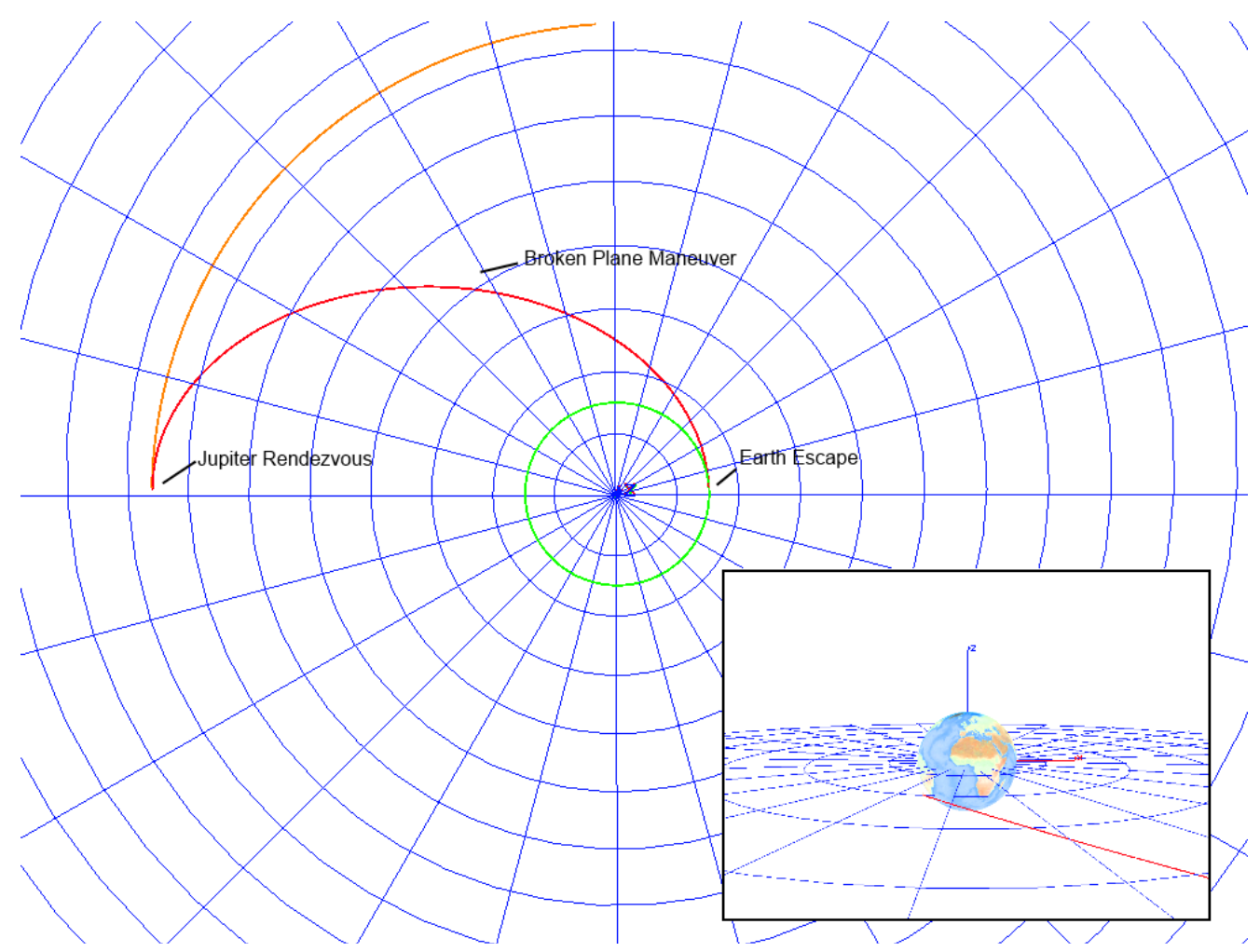

Figure 9: GMAT Plot of the Interplanetary Transfer and Earth Escape

The mission is further detailed below in Table 13, a final orbit state at first apojove, where this analysis concludes. This state represents the "starting point" for final adjustment to a desired science orbit, starting at the first apojove after capture. Also, the entire mission is chronicled in Table 14 in the form of a mission timetable covering the events from Earth SOI escape to the first apojove. Both mission elapsed time (MET) and coordinated universal time (UTC) are given for each event. The full trajectory from Earth escape maneuver to first apojove takes a total of approximately 2.9 years to complete.

Table 13: Achieved Jupiter-Centered Keplerian Elements at First Apojove

\begin{tabular}{lcrr}
\hline Parameter & Symbol & Value & Units \\
\hline \hline Epoch & {$[\mathrm{N} / \mathrm{A}]$} & 30813.663 & $\mathrm{MJD}$ \\
Semi-major Axis & $\mathrm{a}$ & 9823607.400 & {$[\mathrm{~km}]$} \\
Eccentricity & $\mathrm{e}$ & 0.98388 & {$[\mathrm{~N} / \mathrm{A}]$} \\
Inclination & $\mathrm{i}$ & 2.329 & {$[\mathrm{deg}]$} \\
RAAN & $\Omega$ & 8.044 & {$[\mathrm{deg}]$} \\
AOP & $\omega$ & 317.780 & {$[\mathrm{deg}]$} \\
True Anomaly & $\mathrm{N}$ & 180.000 & {$[\mathrm{deg}]$} \\
Perijove & $\mathrm{Rp}$ & 2.215 & {$[\mathrm{RJ}]$} \\
\hline
\end{tabular}


Table 14: Timetable of Important Mission Events

\begin{tabular}{lrr}
\hline Event & MET (T+) & \multicolumn{1}{c}{ UTC Time } \\
\hline \hline Earth Escape Maneuver & 0/00:00:00 & 25 Jun 2022 01:53:32 \\
SC Leaves Earth SOI & 1/03:17:59 & 26 Jun 2022 05:11:31 \\
BPM Performed & $501 / 09: 09: 01$ & 08 Nov 2023 11:02:34 \\
SC Enters Jupiter SOI & $878 / 04: 38: 27$ & 19 Nov 2024 06:31:59 \\
Callisto Periapsis & $957 / 00: 11: 48$ & 06 Feb 2025 02:05:20 \\
Io Periapsis & $958 / 05: 09: 06$ & 07 Feb 2025 07:02:38 \\
JOI Maneuver & $958 / 09: 35: 35$ & 07 Feb 2025 11:29:08 \\
Ganymede Periapsis & $959 / 02: 01: 04$ & 08 Feb 2025 03:54:36 \\
First Apojove & $1058 / 02: 00: 34$ & 18 May 2025 03:54:06 \\
\hline
\end{tabular}

\section{Conclusions}

This approach was designed to function for a variety of initial states leading to a Callisto-Ioperijove-Ganymede flyby sequence. With the inclusion of an optimized broken plane maneuver, the total interplanetary and capture $\Delta \mathrm{V}$ for this mission was $275.8 \mathrm{~m} / \mathrm{s}$, as compared to $330 \mathrm{~m} / \mathrm{s}$ for a Ganymede-Io-JOI double-satellite-aided capture. In the future, the scripts generated here can be used to find additional triple flyby missions to Jupiter by choosing any of the unused initial guesses in different time windows than the one refined here. Additionally, the sequence can be altered to solve for the other triple flyby scenarios such as Callisto-Ganymede-Io triple flybys.

As was shown in this chapter, this particular mission scenario allows for multiple close flybys of Galilean moons, which provide the opportunity to perform large amounts of scientific observation during close approach, especially through the volcanic plumes of Io. Additionally, the triple flyby maneuver allows for a fuel-efficient entry into the Jovian system which has not been accomplished thus far. However, this scenario could greatly benefit from a longer multipleflyby or low-thrust tour of the inner solar system before arrival at Jupiter, akin to the mission of the Juno spacecraft. Further refinement of this mission may include such elements to produce a fully efficient, albeit longer, low-thrust mission from Earth to Jupiter on minimal resources to achieve maximum science. However, the impulsive mission described here requires minimal 
propellant after detachment from the launch vehicle after Earth escape. Furthermore, the elapsed time between Earth escape and Jupiter capture (at Ganymede) is only 2.6 years, which is considerably shorter than comparable mission designs. In the next chapter, the mission described here will be perturbed and the resulting statistical properties will be analyzed. 


\section{Chapter 2: Navigation and Guidance of a Triple Flyby Jovian Capture}

As previously described, satellite-aided captures involve the use of gravity-assist flybys of a satellite or satellites to reduce the orbital energy of a spacecraft such that it becomes captured by the host planet. Sometimes, the spacecraft can be captured solely by the flyby(s), but often an insertion burn is needed to complete the capture. This capture strategy can be compared to typical orbital insertion which closes the incoming hyperbola with a single retrograde burn at periapsis. Satellite-aided capture maneuvers make use of a satellite's large gravity to significantly reduce the magnitude of the insertion burn or eliminate the need for one entirely $[13,33]$. These maneuvers are applicable to any sizable planetary moon system, but are of particular interest in the Jupiter system wherein the four Galilean moons provide large gravityassist potential, are in a predictable resonance pattern, and are of relevant scientific interest [22,34-36]. Double-, triple- and rare quadruple-satellite-aided capture opportunities have been predicted and examined by Lynam [25-27]. A preliminary navigation analysis of double and triple-satellite-aided capture trajectories was performed by Lynam and Longuski [24]. They demonstrated that trajectory correction maneuvers are needed to successfully navigate triplesatellite-aided capture trajectories. Chapter 1 examined and targeted a particular triple-satelliteaided capture trajectory that encountered Jupiter's Galilean moons Callisto, Io and Ganymede, and included a retrograde insertion maneuver at perijove (before the Ganymede encounter) [3738]. This trajectory was modeled without navigation errors and was purely ballistic, except for the main impulsive perijove maneuver. It was found that the Io, and especially Ganymede, flybys were sensitive to initial conditions, and the question of navigational feasibility arose. Similar work by Patrick and Lynam examined a similar maneuver, but with a different Galilean moon encounter sequence, and more importantly, made use of low-thrust solar electric propulsion 
(SEP) [39]. Further such work showed marked improvement, but still suffered from navigational ambiguity that may benefit from the analysis herein described [40].

This chapter further investigates the Callisto-Io-JOI-Ganymede (CIJG) sequence formulated by Didion and Lynam and detailed in the previous chapter [37-38]. Through building a new, original, more flexible model written in MATLAB, the navigational limitations of the NASA General Mission Analysis Tool (GMAT) were eliminated and computation times were decreased. Moreover, access to tailored data, such as the state transition matrix (STM), was facilitated. Spacecraft position and velocity errors were modeled pseudo-randomly along with similar ephemeris knowledge errors pertaining to each Galilean moon. The insertion maneuver was given an error model according to Wagner and Goodson, which was also applied to new trajectory correction maneuvers [41]. The TCMs were used to correct the propagated pseudorandom errors, and each piece of data was carefully collected. An outer Monte Carlo repetition loop ensured that each of thousands of mission simulations were sufficiently randomized and cataloged. This data was then collated and/or averaged for detailed analysis.

\section{A. System Modeling in MATLAB}

The previous GMAT model had much utility in ease of use and detailed graphics, which made it very suitable for prototyping the triple-satellite-aided capture trajectory. However, the built-in features suffered from long computational times and the difficulty in stopping the integrator to extract data, partially due to the complexity of this trajectory. It was decided that a MATLAB model, built in-house and starting from nothing, would be more adaptable and

accessible. With this new model, pseudo-random errors could be injected at specific times and the STM could be carried and used to target TCMs and correct the trajectory. 
A model was built in MATLAB which made use of the ode113 differential equation solver to integrate the seven-body Jovian dynamical system through time. In Jupiter-centered coordinates, the four Galilean moons and the sun propagated according to ephemeris checks for each timestep. The four moons, the sun, and Jupiter formed the gravitational model, with a spacecraft of negligible mass as the seventh body. For reference, the definitions of the B-plane dot-products are reiterated below in Figure 10.

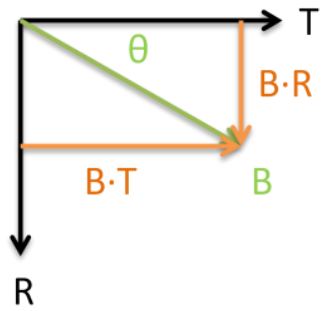

Figure 10: Definition of the B-plane Dot Products

\section{Equations of Motion and Initial State}

The spacecraft began at a similar initial state as previously determined by the GMAT model in Chapter 1. Table 15 below details the Jupiter-centered coordinates, reflecting a slight optimization difference, and targeting a 300 rather than 200 day final period [37-38]. The epoch was February 3, 2025, 02:30:30.595.

Table 15: Spacecraft Initial State, Jupiter-Centered Ecliptic J2000

\begin{tabular}{rccc}
\hline & $\mathbf{x}$ & $\mathbf{y}$ & $\mathbf{z}$ \\
\hline \hline Position $[\mathrm{km}]$ & -4568345.274 & 1030.943 & -60834.882 \\
Velocity $[\mathrm{km} / \mathbf{s}]$ & 9.248 & -1.868 & 0.064 \\
\hline
\end{tabular}

The model was propagated using the 3-body equations of motion as described by Vallado, which were adjusted for seven bodies [42]. This was done simply by adding more terms for each gravitational source and caused negligible additional complexity because it assumes the spacecraft has no gravity of its own and the celestial bodies are "on rails", or do not experience dynamical forces; they only move according to the ephemeris predictions. 


$$
\ddot{\vec{r}}_{J-S C}=-\frac{\mu_{J} \vec{r}_{J-S C}}{\left\|\vec{r}_{J-S C}\right\|^{3}}+\sum_{i=1}^{5} \mu_{i}\left(\frac{\vec{r}_{S C-i}}{\left\|\vec{r}_{S C-i}\right\|^{3}}-\frac{\vec{r}_{J-i}}{\left\|\vec{r}_{J-i}\right\|^{3}}\right)
$$

In Equation 1, "SC" denotes a quantity pertaining to the spacecraft, "J" for Jupiter, and "i" for each third body; e.g. terms such as "SC-i" denote quantities pertaining to a third body in relation to the spacecraft. This equation describes how the spacecraft accelerates in response to the gravity of Jupiter and each of the "third bodies". In this case, the term for a third body was repeated a total of five times using the parameters for the sun and each of the four Galilean moons respectively. This served as the differential equation for the ode113 integrator, solving for the trajectory of the spacecraft with time as it traveled through the system.

\section{The State Transition Matrix}

Each integration step propagated the state of the spacecraft (six elements) as well as an STM which incorporated the errors for each body, allowing for easy propagation of inserted errors as described in the next section. Equation 2 shows how the STM, denoted $\Phi$, updates the system state with respect to the initial state.

$$
\text { state }=\Phi\left(t, t_{0}\right) * \text { state }_{0}
$$

In row form, the array handled by ode113 was a 1x330 row vector, which was reduced to a 1x324 vector after separating the STM from the first six entries that represent the spacecraft state. This could then be easily reshaped into the 18x18 STM (Equation 3), as it was used in the following sections to handle error propagation and B-plane targeting. 


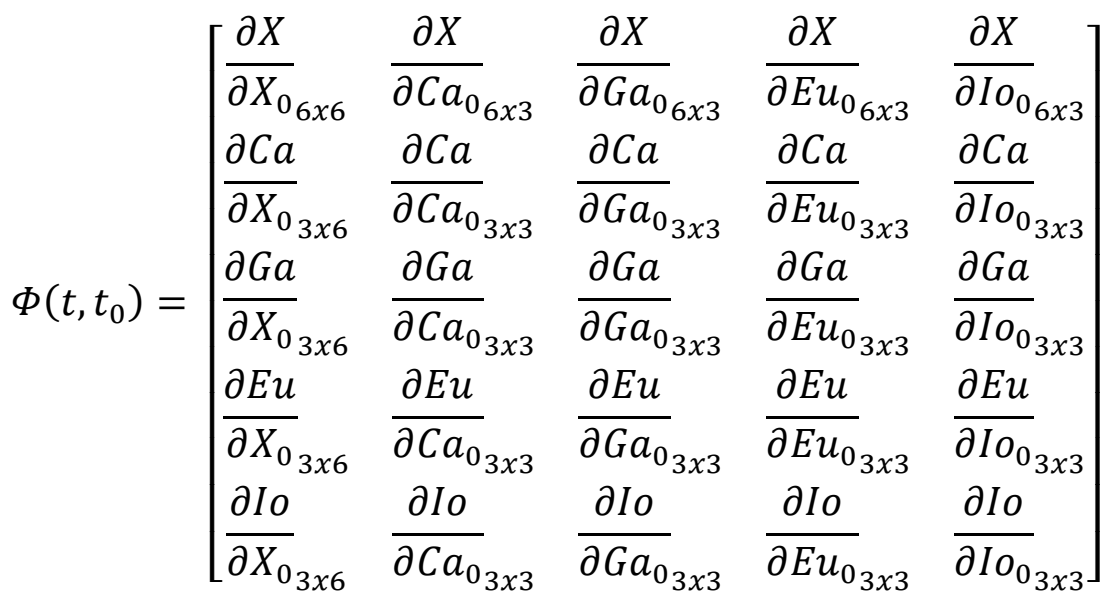

$$
\begin{aligned}
& \frac{\partial X}{\partial X_{0}}=\left[\begin{array}{llllll}
\frac{\partial x}{\partial x_{0}} & \frac{\partial x}{\partial y_{0}} & \frac{\partial x}{\partial z_{0}} & \frac{\partial x}{\partial u_{0}} & \frac{\partial x}{\partial v_{0}} & \frac{\partial x}{\partial w_{0}} \\
\frac{\partial y}{\partial x_{0}} & \frac{\partial y}{\partial y_{0}} & \frac{\partial y}{\partial z_{0}} & \frac{\partial y}{\partial u_{0}} & \frac{\partial y}{\partial v_{0}} & \frac{\partial y}{\partial w_{0}} \\
\frac{\partial z}{\partial x_{0}} & \frac{\partial z}{\partial y_{0}} & \frac{\partial z}{\partial z_{0}} & \frac{\partial z}{\partial u_{0}} & \frac{\partial z}{\partial v_{0}} & \frac{\partial z}{\partial w_{0}} \\
\frac{\partial u}{\partial x_{0}} & \frac{\partial u}{\partial y_{0}} & \frac{\partial u}{\partial z_{0}} & \frac{\partial u}{\partial u_{0}} & \frac{\partial u}{\partial v_{0}} & \frac{\partial u}{\partial w_{0}} \\
\frac{\partial v}{\partial x_{0}} & \frac{\partial v}{\partial y_{0}} & \frac{\partial v}{\partial z_{0}} & \frac{\partial v}{\partial u_{0}} & \frac{\partial v}{\partial v_{0}} & \frac{\partial v}{\partial w_{0}} \\
\frac{\partial w}{\partial x_{0}} & \frac{\partial w}{\partial y_{0}} & \frac{\partial w}{\partial z_{0}} & \frac{\partial w}{\partial u_{0}} & \frac{\partial w}{\partial v_{0}} & \frac{\partial w}{\partial w_{0}}
\end{array}\right]
\end{aligned}
$$

Equation 4 exposes the details of the first block of the STM as written in Equation 3. Here, the spacecraft state (3-D position and velocity) is represented by $\mathrm{X}$, and each Galilean moon is represented by the first two letters of its name. It is important to note that in practice, many of these blocks will become zero or one, while some will contain content of actual use. For example, the correlation between a moon's position and itself is the identity matrix, and that of a moon with another is zero. This means that only the top row of blocks in Equation 3 will have significant content, as they represent the effect of the celestial bodies (and the spacecraft itself) on the spacecraft state. Because the entire problem is set in Jupiter-centered coordinates, the 
partial derivative term represented by Equation 4 not only embodies the motion of the spacecraft, but also the influence on it by Jupiter's gravity well.

\section{B. Insertion of Errors \& Corrections}

The trajectory was split into mission "phases", defined as branches between specific points of interest at which the integration paused. In the scheme used here, the pauses represented an instant in which several simultaneous, instantaneous processes took place. Pauses were enforced by an "events" function inserted into the ode113 options, and took place at critical points such as periapsides, as follows: initial state, Callisto periapsis, Io periapsis, Jupiter periapsis, Ganymede periapsis, arbitrary point outside Ganymede's influence, Jupiter apoapsis. At each pause, the spacecraft gained instantaneous knowledge of its state and the bodies around it, which it used to target TCMs and correct the next phase of the trajectory when appropriate. Once targeted, the TCMs were instantaneously executed before the end of the pause. All of these pieces of knowledge, as well as the TCM execution itself, were subject to error, as is explained in the following sub-sections.

\section{State Errors, Ephemeris Errors, and Delta-DOR}

At each pause, appropriate state errors were determined for the situation before integration resumed; these represent knowledge errors in the spacecraft's position and velocity as well as ephemeris errors for the position of each Galilean moon. The errors were implemented using a call of "randn" for each value each time, multiplying the randn result by a "reasonable" standard deviation value for each error, as detailed below in Table 16. It was assumed that radiometric navigation could provide sufficient trajectory knowledge to reduce the initial state uncertainty to that level. The errors on the moons' positions represent the uncertainty of our present ephemeris knowledge of the Galilean moons. 
Table 16: Standard Deviations for State Errors

\begin{tabular}{l|ccc|ccc}
\hline & \multicolumn{3}{|c|}{ Position [km] } & \multicolumn{3}{c}{ Velocity [mm/s] } \\
SC & $\delta \mathbf{x}$ & $\boldsymbol{\delta y}$ & $\boldsymbol{\delta z}$ & $\boldsymbol{\delta}$ & $\boldsymbol{\delta} \mathbf{v}$ & $\boldsymbol{\delta} \mathbf{w}$ \\
\hline \hline Initial State & 3.0 & 1.0 & 3.0 & 1.0 & 1.0 & 1.0 \\
Other & 0.1 & 0.1 & 0.1 & 1.0 & 1.0 & 1.0 \\
\hline Moons & & & & & & \\
\hline Departure & 0.1 & 0.1 & 0.1 & & & \\
Arrival & 5.0 & 5.0 & 5.0 & & & \\
\hline
\end{tabular}

For each pause, there were six errors applied to the spacecraft state and three position errors applied to each applicable moon. The spacecraft state error was assumed to be large at the initial state and an order of magnitude smaller at all other pauses. At each pause, the values in the lower half of Table 16 detail the smaller errors at the moon currently being departed and larger errors for the next to be encountered, e.g. at the pause at Callisto periapsis, there is a small error on Callisto's position and a large error on Io's position. Likewise, the pause at Ganymede periapsis experienced small error in Ganymede's position, but no "arrival" error, because the next pause point is in open Jovian space. Once a vector of reasonable errors was determined, it was multiplied by the propagated STM from the previous integration and the spacecraft state was updated to reflect the error. This new state was the state plotted and used for the next integration.

Note that the $\delta \mathrm{x}$ and $\delta \mathrm{z}$ component of initial state position error are three times the $\delta \mathrm{y}$ component. This error represents the influence of error inherent in Delta-Differential One-Way Ranging (Delta-DOR) navigation for the interplanetary phase. Delta-DOR ranging, which uses the Deep Space Network (DSN) for Very-Long-Baseline Interferometry (VLBI), is subject to more error in the plane normal to the vector connecting Earth and the spacecraft, and much less in the range direction. This measurement is performed by measuring the angular movement of the spacecraft and Earth, and comparing against a stationary distant celestial object, like a quasar. Below, Figure 11 shows a graphical representation of this method. 


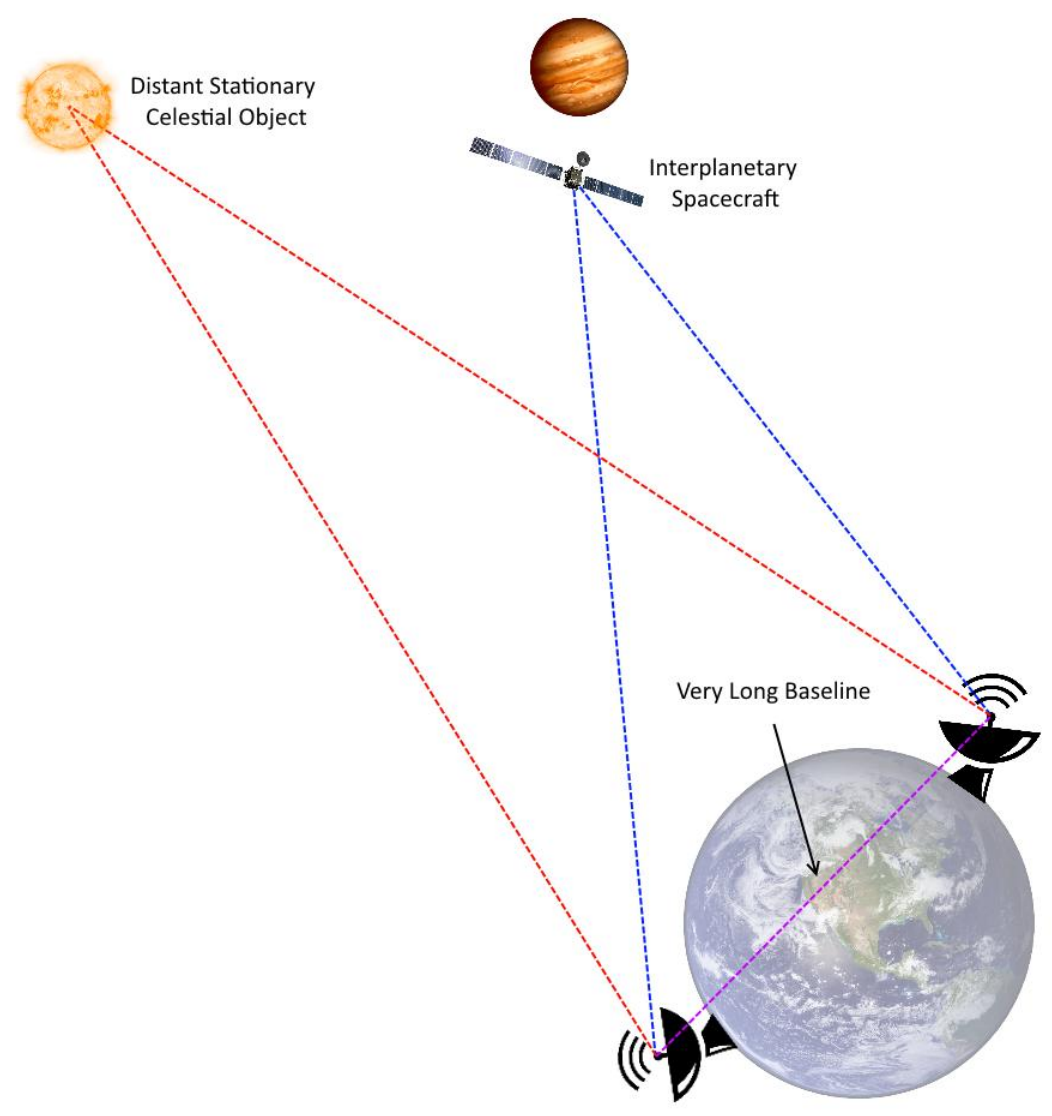

Figure 11: Example Delta-DOR Setup

Current techniques, as described by Curkendall and Border, boast the ability to reduce DeltaDOR errors to less than one nanoradian (in ideal conditions) and around two nanoradians consistently, which translate to approximately $0.8 \mathrm{~km}$ and $1.6 \mathrm{~km}$ respectively at the range in question [43]. This value was conservatively increased to 2.5 nanoradians for this analysis, or about $2 \mathrm{~km}$. This is added to an optimistic $1 \mathrm{~km}$ in Jupiter ephemeris error, which assumes the successful characterization of the Jupiter system ephemeris by Juno, to produce the tabulated values.

\section{B-Plane Targeting \& TCMs}

At Callisto periapsis, a TCM was used to ensure the next arrival at Io was in the required area of the Io B-plane in order to achieve the desired flyby, i.e. matching the desired B-plane dot 
products: $B \cdot T$ and $B \cdot R$. Here $\mathrm{S}$ is the B-plane normal, T corresponds to the moon's equator, normal to $\mathrm{S}$ and in the B-plane, and $R=S \times T$. Likewise, the composite JOI maneuver was targeted to properly intercept Ganymede's B-plane. To achieve this, the Callisto periapsis pause and the JOI pause featured B-plane targeting loops which would apply a "guess" TCM, propagate until arrival, calculate the B-plane, and repeat with a new TCM depending on an objective function until agreement with desired values was reached. The objective function logic is explained below in Equations 5-8, given the B-plane dot products and derivatives from geometry, where $\Phi$ is the STM as previously described and X is the spacecraft state.

$$
\begin{gathered}
F=\left[\begin{array}{l}
B \cdot R_{a c t}-B \cdot R_{\text {nom }} \\
B \cdot T_{a c t}-B \cdot T_{\text {nom }}
\end{array}\right] \\
\frac{\partial X}{\partial u}=\Phi(1: 6,4), \quad \frac{\partial X}{\partial v}=\Phi(1: 6,5), \quad \frac{\partial X}{\partial w}=\Phi(1: 6,6) \\
D F=\left[\begin{array}{lll}
\frac{\partial(B \cdot R)}{\partial X} * \frac{\partial X}{\partial u} & \frac{\partial(B \cdot R)}{\partial X} * \frac{\partial X}{\partial v} & \frac{\partial(B \cdot R)}{\partial X} * \frac{\partial X}{\partial w} \\
\frac{\partial(B \cdot T)}{\partial X} * \frac{\partial X}{\partial u} & \frac{\partial(B \cdot T)}{\partial X} * \frac{\partial X}{\partial v} & \frac{\partial(B \cdot T)}{\partial X} * \frac{\partial X}{\partial w}
\end{array}\right] \\
\Delta V=T C M_{1}=T C M_{0}-D F^{T} /\left(D F^{T} * D F\right) * F
\end{gathered}
$$

In Equation 5, an objective function $\mathrm{F}$ was set up, representing the difference between the desired, nominal (nom) B-plane dot products and the actual values achieved. This objective function ultimately determined when the target was reached and the targeting loop could end. Equation 6 represented state derivatives as extracted from the STM, which were needed to take the derivative of F, as calculated in Equation 7. Finally, in Equation 8, the TCM was updated with a new "guess", according to a function of the derivative of the objective function with the 
change in TCM components. This logic then repeated, integrating from the same state with the new TCM, calculating the B-plane parameters each time until both values of the F matrix were lower than an applied tolerance; in this case, the tolerance used was one meter. This entire process served to both target the necessary ideal TCMs and to correct position and velocity error as inserted previously. However, these maneuvers were still subject to error themselves, as described in the next sub-section.

\section{Maneuver Execution Error Modeling}

Maneuver execution error was applied onto each TCM as well as the JOI maneuver and was based on the maneuver error model analyzed by Wagner and Goodson [41]. This model provides proportional and fixed error magnitudes describing Cassini's main engine assembly (MEA) and reaction control system (RCS) propulsion methods. The error for TCMs 1 and 2 (Callisto periapsis and post-Ganymede respectively) were modeled using the RCS values, while the JOI composite maneuver was modeled using the MEA values as shown in Table 17. These values represent the standard deviation values and errors are applied by multiplying a randn call by the model equation (Equation 9) and adding them vectorially onto the ideal maneuvers. The equation is also given below, with $\sigma_{1}$ being the fixed values, $\sigma_{2}$ being the proportional values, and y being the magnitude of the nominal maneuver.

Table 17: Cassini 2007-02 Maneuver Execution Error Model [39]

\begin{tabular}{l|l|rr}
\hline \multicolumn{2}{|l}{} & MEA & RCS \\
\hline \hline Magnitude & Proportional [\%] & 0.02 & 1.2 \\
& Fixed [mm/s] & 5.0 & 0.8 \\
Pointing & Proportional [mrad] & 0.6 & 5.5 \\
(per axis) & Fixed [mm/s] & 3.0 & 0.0 \\
\hline
\end{tabular}




$$
d \overrightarrow{\Delta V}=\operatorname{randn} * \sqrt{\sigma_{1}^{2}+y^{2}{\sigma_{2}}^{2}}
$$

This analysis leads to the following: Equations 10-13, with "mag", "point1", and "point2" forming an orthogonal set representing the magnitude error, in the direction of the nominal maneuver, and two pointing errors, oriented at right angles to the maneuver direction, $\Delta \widehat{V}$. These equations are used similarly when calculating the TCM errors with the RCS values, wherein the "УлoI" would be replaced by "УтСм", the magnitude of the nominal TCM, etc.

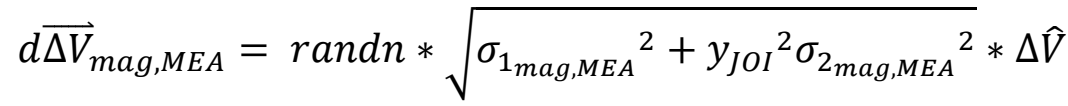

$$
\begin{aligned}
& d \overrightarrow{\Delta V}_{\text {point } 1, M E A}=\operatorname{rand} n * \sqrt{{\sigma_{1_{\text {point }, M E A}}{ }^{2}+y_{J O I}{ }^{2}{\sigma_{2 o i n t, M E A}}^{2}}_{\Delta}^{2}} * \Delta \widehat{V}_{p 1} \\
& d \overrightarrow{\Delta V}_{\text {point } 2, M E A}=\operatorname{rand} n * \sqrt{{\sigma_{1_{\text {point }, M E A}}{ }^{2}+y_{J O I}{ }^{2}{\sigma_{2 \text { point }, M E A}}^{2}}_{\Delta}} * \Delta \widehat{V}_{p 2} \\
& d \overrightarrow{\Delta V}_{M E A}=d \overrightarrow{\Delta V}_{m a g, M E A}+d \overrightarrow{\Delta V}_{\text {point } 1, M E A}+d \overrightarrow{\Delta V}_{\text {point } 2, M E A}
\end{aligned}
$$

In these equations, the unit vectors can be described by relations in Equation 14, where $\hat{z}$ is the vertical direction unit vector.

$$
\Delta \widehat{V}=\frac{\overrightarrow{\Delta V}}{\|\overrightarrow{\Delta V}\|}, \quad \Delta \widehat{V}_{p 1}=\Delta \widehat{V} \times \hat{z}, \quad \Delta \widehat{V}_{p 2}=\Delta \widehat{V} \times \Delta \widehat{V}_{p 1}
$$

The result of Equation 14 was then added onto the nominal maneuver and propagation continued. This occurred using the appropriate values from Table 17 for each of the two TCMs and for the adjusted JOI composite maneuver. 


\section{Statistical Modeling via Monte Carlo Simulation}

In order to determine the stochastic robustness of this mission profile and ultimately determine its average sensitivity to reasonable perturbations, a Monte Carlo simulation scheme was developed to repeat the MATLAB script several thousand times, each with new pseudorandom perturbations. This scheme was implemented by creating a simple frame script which randomizes the "randstream" seed variable based on the current clock time and the mt19937ar (Mersenne Twister) algorithm, ensuring that each repeated run receives legitimately different pseudo-random values for continued calls of randn. Without this measure, it would be unclear whether the randstream was being reused for continued runs conducted in a single session. The repetition script set up a while loop which ran until a set time of day (usually overnight), repeatedly running the simulation function and appending a vector of mission data onto a large array. This large array was then saved into a .mat file and an excel spreadsheet for thorough statistical post-processing.

\section{Results of MATLAB Trajectory Modeling}

This section discusses the outcome of performing the triple-satellite-aided capture sequence in the complete Jovian system model in MATLAB. First, data is presented to verify that without perturbations, a nominal trajectory through this sequence matched, within reasonable tolerance, the GMAT trajectory of the same initial conditions and given maneuvers. Second, the results of the Monte Carlo repetition of the script, with pseudo-random perturbations injected at the appropriate points, are presented and discussed. Data on the statistical distributions of physical trajectory parameters are presented, with graphical representations of each of the individual moons' flyby B-planes. 


\section{Verification of the MATLAB Model \& Nominal Characteristics}

The new MATLAB model was verified for accuracy against the previously used GMAT model before proceeding. This was done by giving the MATLAB model the full set of initial spacecraft conditions, the set JOI magnitude, and the ephemeris epoch used in the GMAT model. The spacecraft was then allowed to freely propagate through the nominal trajectory without any perturbations, targeting, or trajectory correction. At the end, the position of the spacecraft during flybys was compared to the GMAT model. The radial error at Callisto was $0.10 \%$, which when left to ballistically propagate without errors or correction led to a $2.67 \%$ radial error at Ganymede closest approach. The final period was 295.271 days, within 5 days of the 300 day target. This error was deemed acceptable, and analysis continued with the new MATLAB model. Below, in Table 18, are the "expected" nominal trajectory parameters for the mission (from GMAT), against which will later be compared the average values of the Monte Carlo simulation.

Table 18: Expected Nominal Parameters from GMAT Model

\begin{tabular}{lrr}
\hline Parameter & Value & Unit \\
\hline \hline Callisto Alt. & 505.0 & {$[\mathrm{~km}]$} \\
Io Alt. & 282.0 & {$[\mathrm{~km}]$} \\
Ganymede Alt. & 98.5 & {$[\mathrm{~km}]$} \\
Perijove Radius & 3.3 & {$[\mathrm{RJ}]$} \\
JOI & 253.0 & {$[\mathrm{~m} / \mathrm{s}]$} \\
Final Period & 300.0 & {$[\mathrm{day}]$} \\
\hline
\end{tabular}

\section{Monte Carlo Statistical Characteristics}

Using the Monte Carlo repetition frame script previously described, a total of 1887 mission runs were completed and all relevant values were catalogued. Brief post-processing of the data in both Excel and MATLAB confirmed that of the 1887 runs, not a single case resulted in failure, i.e., no run failed to converge, and no trajectory intersected the surface of a moon, used excessive $\Delta \mathrm{V}$, or failed to achieve the desired 300 day final orbital period. Furthermore, the data collected 
was analyzed to extract the statistical properties of each individual quantity of interest, including the minimum, maximum, range, and standard deviation of each flyby radius/altitude, B-plane parameters, final period, and TCM and JOI magnitudes. Below in Figure 12 is an example trajectory, a single run through the triple-satellite-aided capture sequence showing the locations of each of the flybys at the time of closest approach, apojove is not shown. All integration pauses are marked with an $\mathrm{X}$, moons with a circle, and Jupiter with a star. Note the post-Ganymede TCM pause that does not coincide with a flyby, and the JOI pause at perijove. On this scale, all of the Monte Carlo runs would look exactly like Figure 12. Figure 13 is the very same trajectory, but zoomed to more precisely detail the individual mission phases.

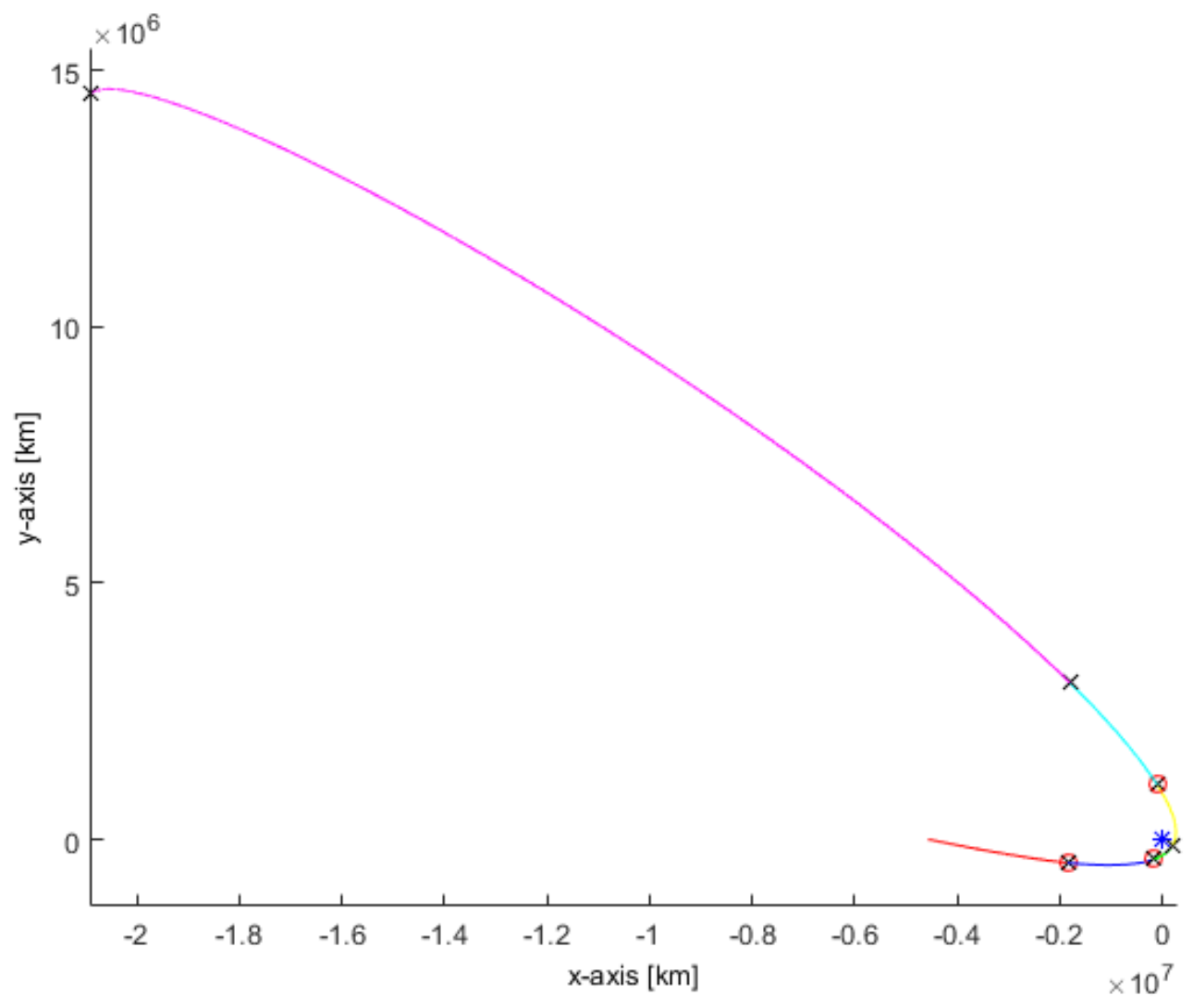

Figure 12: Example Callisto-Io-Ganymede Trajectory, Jupiter-Centered Coordinates 


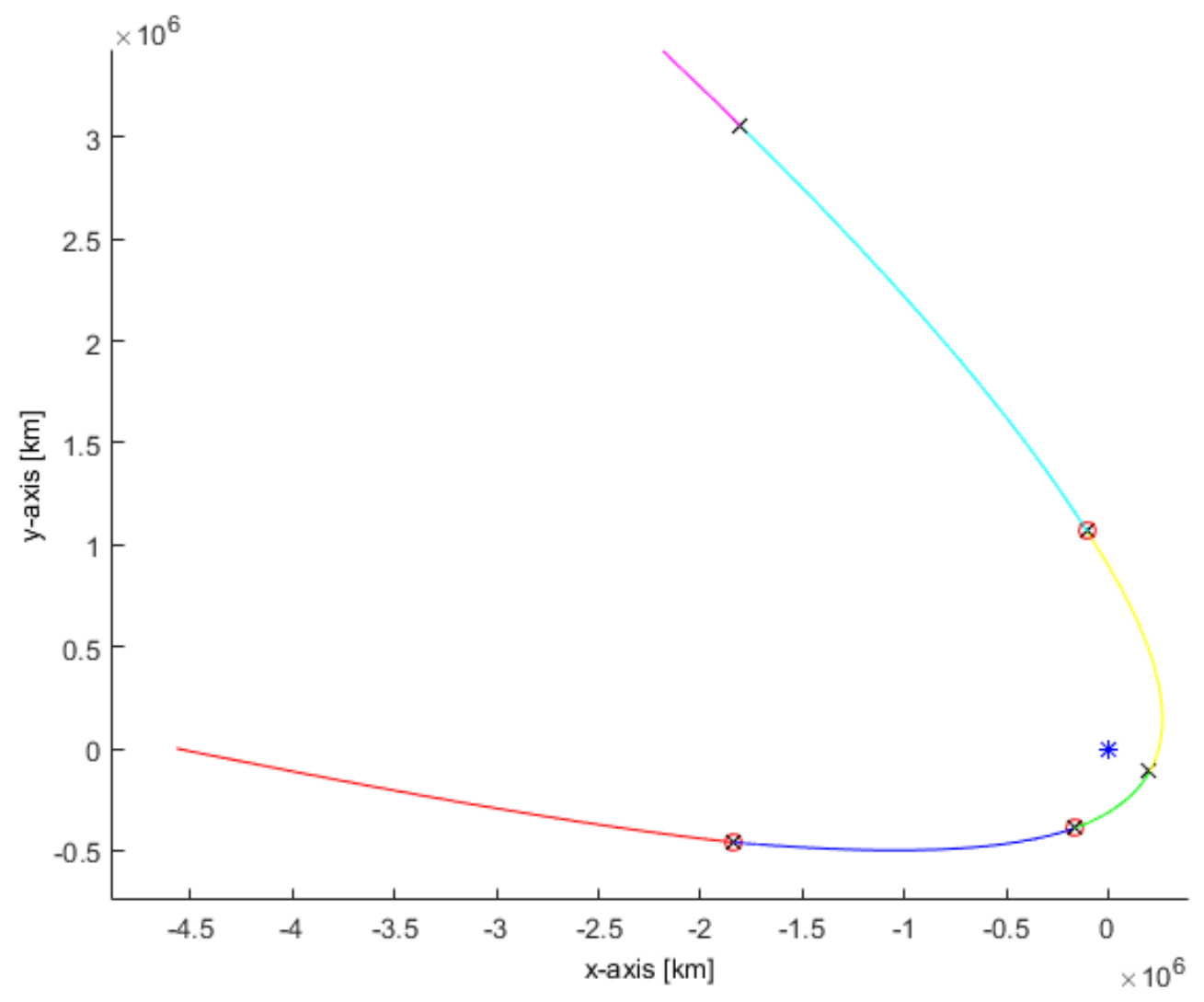

Figure 13: Example Callisto-Io-Ganymede Trajectory, Jupiter-Centered Coordinates, Zoomed

Because the initial state was subject to errors in the positions and velocities of Jupiter, Callisto, and the spacecraft, and it did not receive a TCM before the ballistic approach to Callisto, the Callisto flyby experienced the greatest spread in B-plane parameter error. However, the errors experienced were still relatively small, and the Callisto periapsis TCM was performed afterwards, beginning the navigation and ensuring smaller errors for the subsequent mission phases. Below, Table 19 details the statistical properties of the Callisto flyby, while Figure 14 gives a graphical scatter plot showing the B-plane intersection point spread for the 1887 script repetitions. 
Table 19: Callisto Flyby Statistical Properties

\begin{tabular}{|c|c|c|c|c|c|}
\hline & $\mathbf{R p}$ & Altp & BdotR & BdotT & B Angle \\
\hline Average & 2918.20 & 507.90 & -2.21 & 2962.57 & -0.04 \\
\hline Min. & 2899.49 & 489.19 & -19.96 & 2943.86 & -0.39 \\
\hline Max & 2936.18 & 525.88 & 15.91 & 2980.55 & 0.31 \\
\hline Range & 36.69 & 36.69 & 35.88 & 36.69 & 0.69 \\
\hline Std. Dev. & 5.29 & & 5.69 & 5.29 & 0.11 \\
\hline & {$[\mathrm{km}]$} & {$[\mathrm{km}]$} & {$[\mathrm{km}]$} & {$[\mathrm{km}]$} & [deg] \\
\hline
\end{tabular}

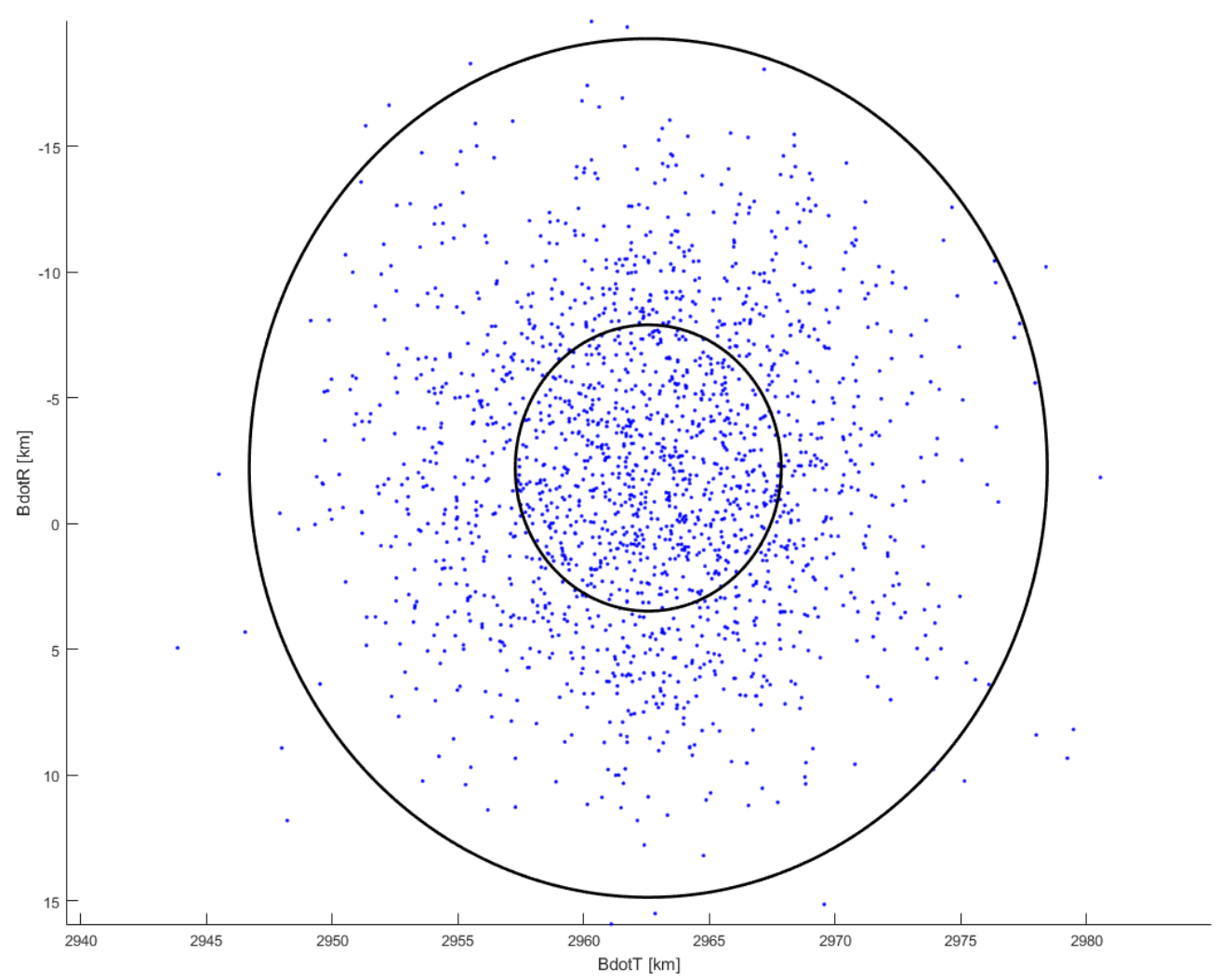

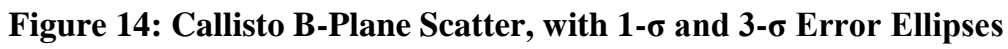

Note from Table 20 that the range in Callisto flyby altitude was still only $36.69 \mathrm{~km}$, with a very safe minimum altitude of $489.19 \mathrm{~km}$. Furthermore, the B-plane angle achieves an average of $-0.04 \mathrm{deg}$, very close to the desired 0 degree planar flyby. Similarly, the Io flyby was analyzed to reveal its statistical distribution and B-plane spread. Table 20 and Figure 15 below detail these results. 
Table 20: Io Flyby Statistical Properties

\begin{tabular}{|c|c|c|c|c|c|}
\hline & $\mathbf{R p}$ & Altp & BdotR & BdotT & B Angle \\
\hline Average & 2112.70 & 291.10 & -964.54 & 1902.65 & -26.88 \\
\hline Min. & 2093.82 & 272.22 & -983.64 & 1884.19 & -27.37 \\
\hline Max & 2129.44 & 307.84 & -946.15 & 1917.99 & -26.39 \\
\hline Range & 35.62 & 35.62 & 37.49 & 33.80 & 0.98 \\
\hline Std. Dev. & 5.19 & & 5.25 & 5.19 & 0.14 \\
\hline & {$[\mathrm{km}]$} & {$[\mathrm{km}]$} & {$[\mathrm{km}]$} & {$[\mathrm{km}]$} & {$[\mathrm{deg}]$} \\
\hline
\end{tabular}

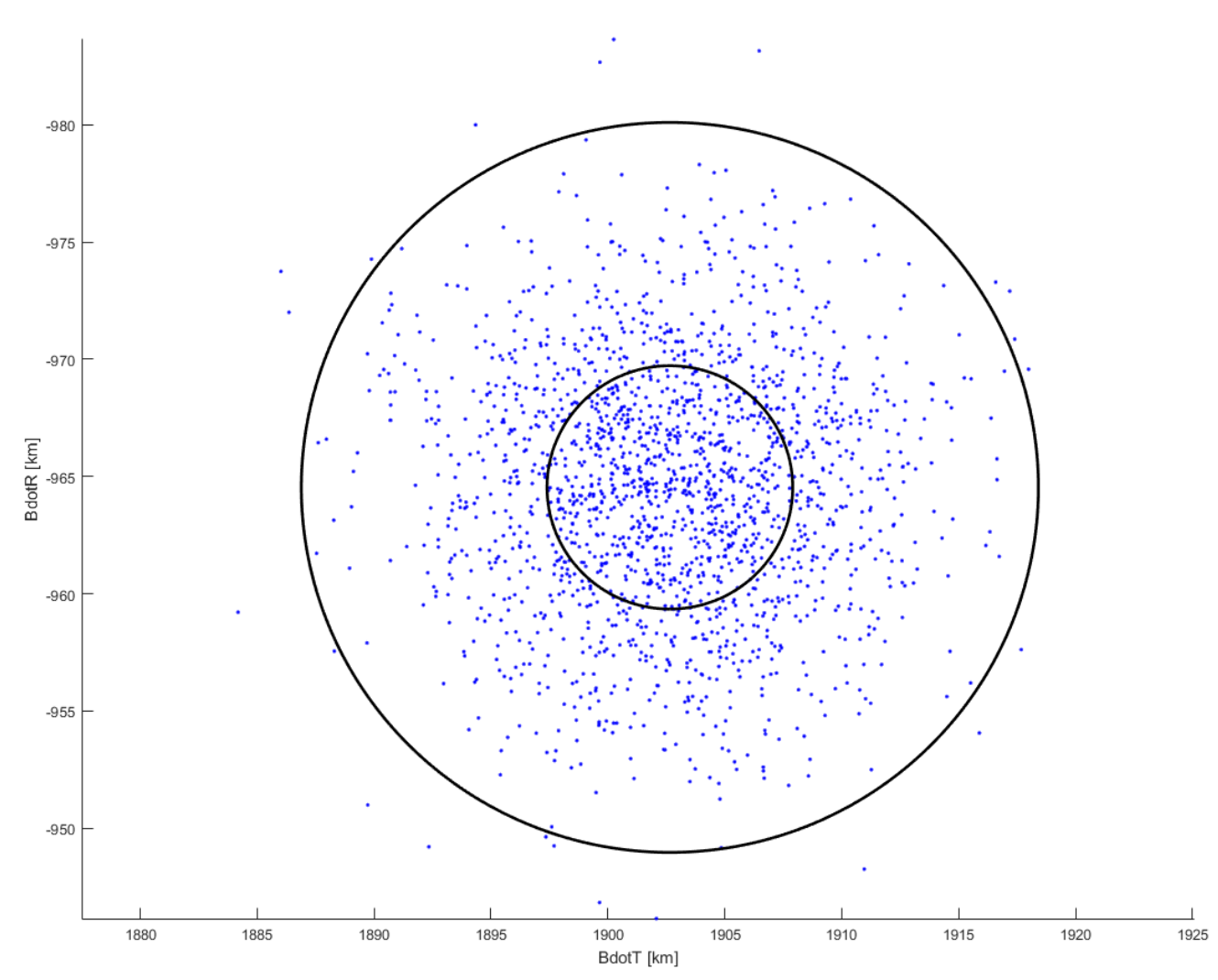

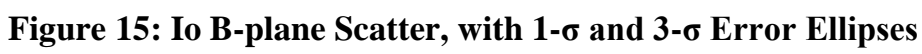

Because the Callisto periapsis TCM served to target the Io flyby, the spread here was much more controlled, with an altitude range of $35.62 \mathrm{~km}$ and a safe minimum altitude of $272.22 \mathrm{~km}$. Note that the Callisto periapsis TCM targeted a specific Io B-plane angle in order to reach a suitable inclination to reach Ganymede. The angle here was very consistent around the average of -26.88 deg with a range of only $0.98 \mathrm{deg}$. A similar set of data is presented for the Ganymede flyby, after the composite JOI maneuver, below in Table 21 and Figure 16. 
Table 21: Ganymede Flyby Statistical Properties

\begin{tabular}{|c|c|c|c|c|c|}
\hline & $\mathbf{R p}$ & Altp & BdotR & BdotT & B Angle \\
\hline Average & 2729.70 & 98.50 & 512.47 & -2731.34 & 169.37 \\
\hline Min. & 2709.88 & 78.68 & 494.28 & -2752.09 & 168.94 \\
\hline Max & 2749.70 & 118.50 & 532.11 & -2711.10 & 169.74 \\
\hline Range & 39.82 & 39.82 & 37.83 & 40.99 & 0.80 \\
\hline Std. Dev. & 5.69 & & 5.01 & 5.75 & 0.10 \\
\hline & {$[\mathrm{km}]$} & {$[\mathrm{km}]$} & {$[\mathrm{km}]$} & {$[\mathrm{km}]$} & {$[\mathrm{deg}]$} \\
\hline
\end{tabular}

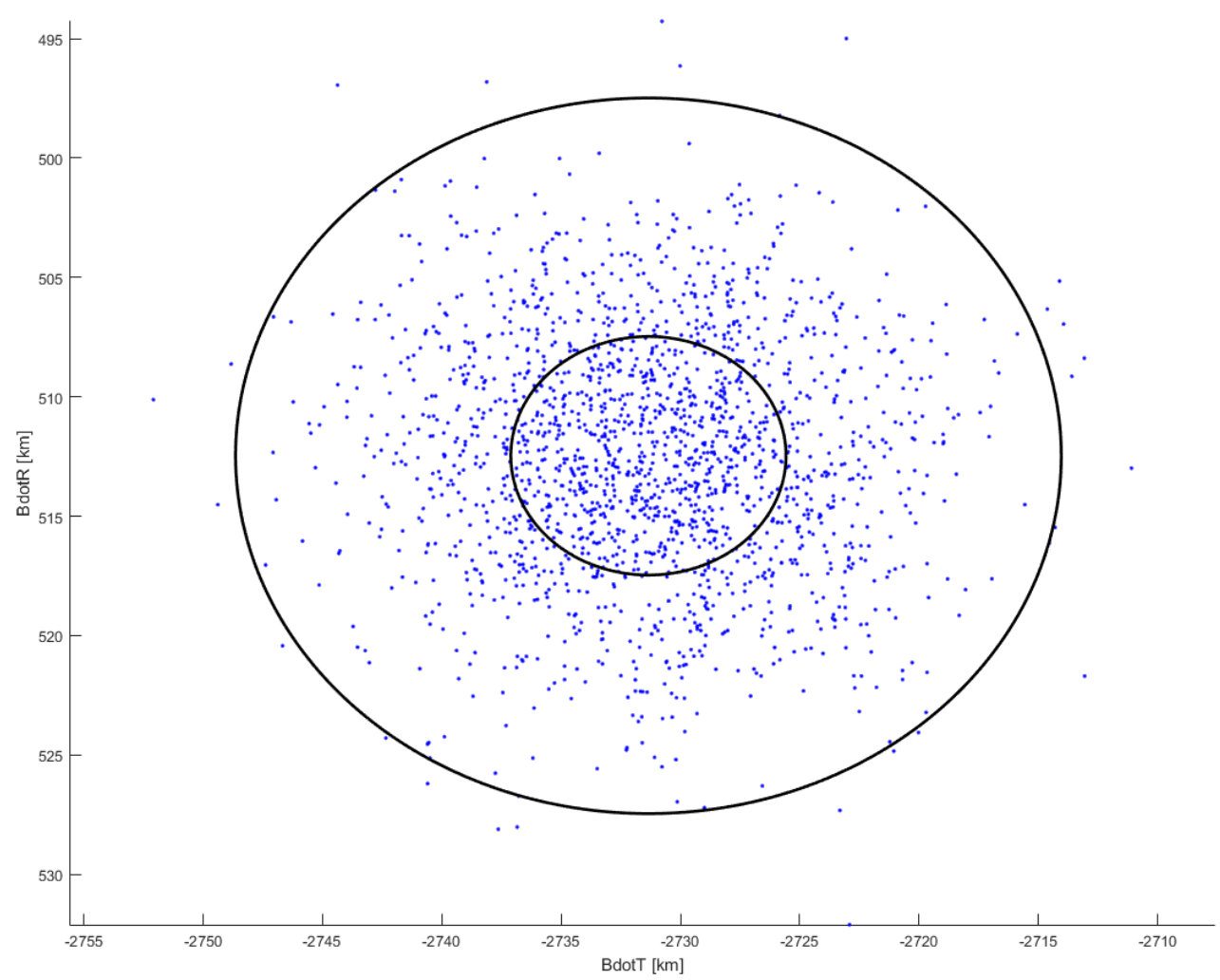

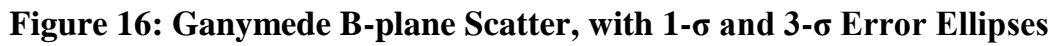

The Ganymede flyby spread is relatively controlled in $B \cdot R$, but varies slightly more in $B \cdot T$, with ranges of $37.83 \mathrm{~km}$ and $40.99 \mathrm{~km}$ respectively, lending to a noticeable oblateness in the error ellipses. This is, however, not a significant issue because the Ganymede flyby is not critical for targeting subsequent flybys like the previous ones. The flyby is successful as it receives sizable gravity assist without approaching dangerously close; and the following TCM 2 can easily correct these errors to target the appropriate apojove. TCM 2 is essentially a JOI cleanup 
maneuver, so it is not required to safely navigate the flybys. However, the subsequent Jupiter satellite tour would require it to efficiently target the first flyby after capture.

The script recorded all of the components for each maneuver, and through post-processing the statistical properties were extracted for each component. Additionally, the mission $\Delta \mathrm{V}$ was recorded and analyzed similarly. The reduced statistical maneuver data can be examined below in Table 22.

Table 22: Statistical Maneuver Data $[\mathrm{m} / \mathrm{s}]$

\begin{tabular}{|c|c|c|c|c|c|c|c|c|c|}
\hline & \multicolumn{3}{|c|}{ TCM 1 (Callisto periapsis) } & \multicolumn{2}{|c|}{ Nominal JOI } & \multirow[b]{2}{*}{$\Delta \mathbf{V z}$} & \multicolumn{3}{|c|}{ JOI Adjustment } \\
\hline & $\Delta \mathbf{V} x$ & $\Delta \mathbf{V y}$ & $\Delta \mathbf{V z}$ & $\Delta \mathbf{V} x$ & $\Delta V y$ & & $\Delta \mathbf{V x}$ & $\Delta \mathbf{V y}$ & $\Delta \mathbf{V z}$ \\
\hline Average & 0.003 & -0.001 & 0.010 & -122.616 & -221.027 & -8.767 & -1.217 & -0.388 & 2.695 \\
\hline Min. & -0.948 & -0.243 & -1.456 & -122.689 & -221.070 & -8.770 & -13.536 & -2.873 & 1.216 \\
\hline Max. & 1.012 & 0.196 & 1.370 & -122.538 & -220.986 & -8.763 & 10.225 & 1.888 & 4.110 \\
\hline Range & 1.960 & 0.439 & 2.827 & 0.151 & 0.083 & 0.007 & 23.761 & 4.761 & 2.894 \\
\hline \multirow[t]{3}{*}{ Std. Dev. } & 0.319 & 0.070 & 0.466 & 0.022 & 0.012 & 0.001 & 3.707 & 0.737 & 0.431 \\
\hline & \multicolumn{3}{|c|}{ TCM 2 (post-Ganymede) } & \multicolumn{3}{|c|}{ Apojove Period Match } & \multirow{2}{*}{\multicolumn{3}{|c|}{$\begin{array}{l}\text { Mission } \Delta V \\
\text { Magnitude } \\
\end{array}$}} \\
\hline & $\Delta \mathbf{V x}$ & $\Delta \mathbf{V y}$ & $\Delta \mathbf{V z}$ & $\Delta \mathbf{V x}$ & $\Delta \mathbf{V y}$ & $\Delta \mathbf{V z}$ & & & \\
\hline Average & -0.070 & 0.163 & 0.011 & -0.002 & -0.004 & 0.000 & & 264.023 & \\
\hline Min. & -12.601 & -33.945 & -1.755 & -0.642 & -0.906 & -0.089 & & 254.009 & \\
\hline Max. & 11.190 & 36.649 & 1.667 & 0.616 & 0.851 & 0.094 & & 302.761 & \\
\hline Range & 23.790 & 70.595 & 3.422 & 1.257 & 1.757 & 0.183 & & 48.753 & \\
\hline Std. Dev. & 3.677 & 11.232 & 0.507 & 0.207 & 0.286 & 0.025 & & 7.740 & \\
\hline
\end{tabular}

This table details the average, minimum, maximum, range, and standard deviations of each component of each maneuver in $\mathrm{m} / \mathrm{s}$. It is important to note that these are the bounds of each component, and do not correspond to a particular maneuver instance. A histogram showing mission $\Delta \mathrm{V}$ magnitude probability distribution can be examined in Figure 17. 


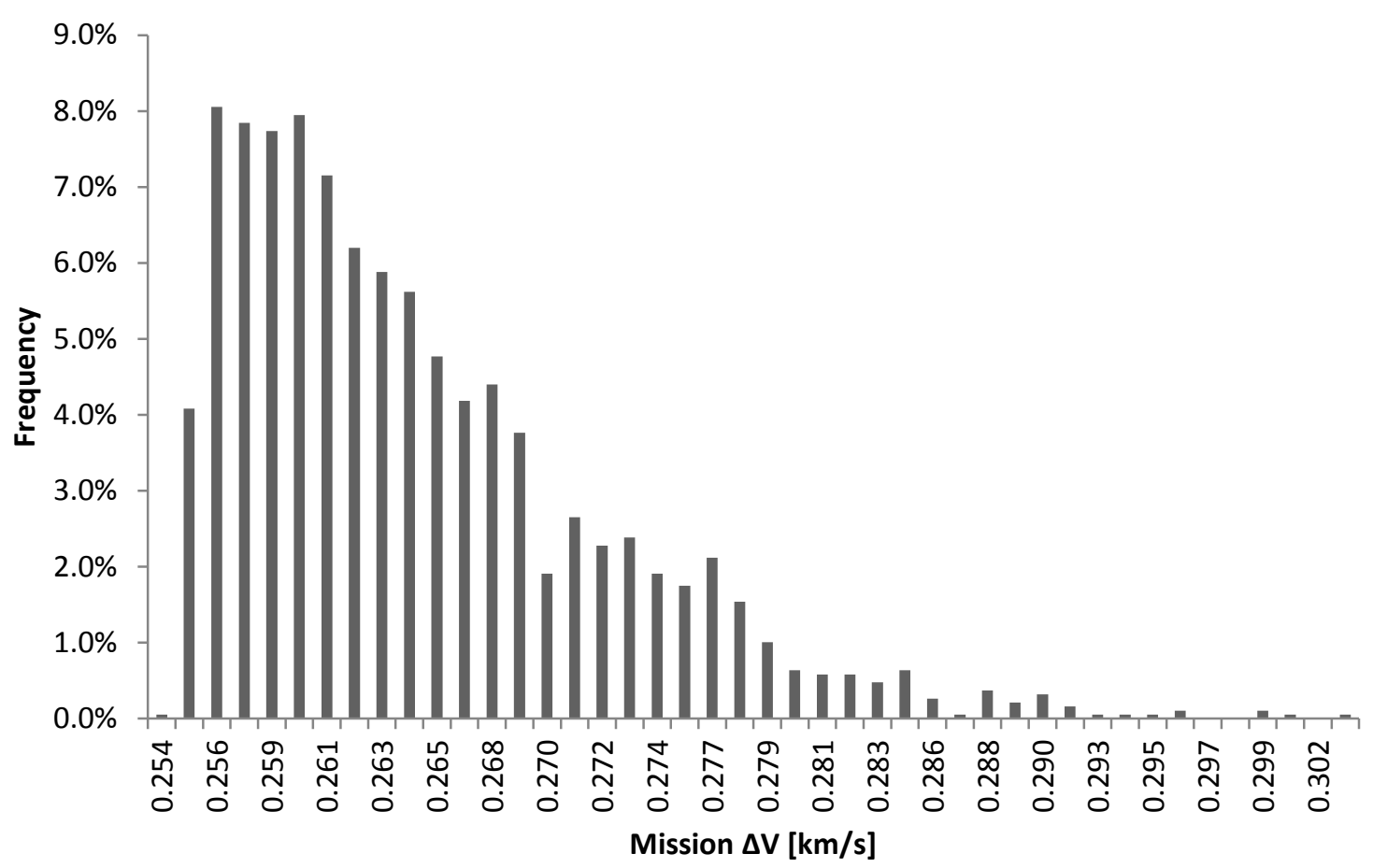

Figure 17: Mission $\Delta \mathrm{V}$ Histogram

As is shown, the mission $\Delta \mathrm{V}$ probability distribution was heavily skewed to the low end, but covered a sizable range, from a minimum of $254.009 \mathrm{~m} / \mathrm{s}$ to the $99^{\text {th }}$ percentile value of 288.938 $\mathrm{m} / \mathrm{s}$ (with a few outliers above that value). However, the average was $264.023 \mathrm{~m} / \mathrm{s}$, the median was $262.096 \mathrm{~m} / \mathrm{s}$, and the probability distribution peaked at an expected mission $\Delta \mathrm{V}$ value of $256.276 \mathrm{~m} / \mathrm{s}$, representing 152 runs, approximately $8 \%$ of the total 1887 . This further demonstrates the trend toward a favorably low mission $\Delta \mathrm{V}$, with the extreme maximum having a very rare chance of occurrence when unfavorable perturbations combined, resulting in only two of the 1887 runs requiring a $\Delta \mathrm{V}$ of more than $300 \mathrm{~m} / \mathrm{s}$. Furthermore, because the ideal postGanymede TCM experienced no execution error, it had no issue achieving the 295.271 day period of the nominal MATLAB trajectory, with a range and standard deviation on the order of the machine's precision due to its deterministic behavior. 


\section{E. Conclusions}

As a general measure of success, the Monte Carlo simulation experienced no fatal script errors and no instances of a sub-surface flyby. The statistical properties of the mission $\Delta \mathrm{V}$ requirements were consistent and favorable, demonstrating that the mission is feasibly navigable given reasonable errors. Perturbations did not, in any case, cause extreme course deviations that cannot be corrected with modest trajectory corrections. As will be discussed in the following sections, the results fall within expected boundaries and represent an efficient, desirable mission design for Jovian capture which can be used for missions with various means and ends. The method employed is rigorous and adjustable, statistically stable and theoretically sound. The method can be altered at nearly any time, and even the results described herein represent a marginal improvement upon the original published results [44].

\section{1. $\Delta V$ Comparison}

The flyby sequence examined here was a triple-satellite-aided capture with a perijove radius of approximately $3.3 \mathrm{RJ}$, yielding a median mission $\Delta \mathrm{V}$ of $262.096 \mathrm{~m} / \mathrm{s}$ and median adjusted JOI magnitude of $253.709 \mathrm{~m} / \mathrm{s}$. This approximately fits in the preliminary navigation findings of Lynam and Longuski, as shown by their representative data shown in Table 23, which claimed a best-case triple-flyby JOI of $245 \mathrm{~m} / \mathrm{s}$ with a perijove of $3 \mathrm{RJ}$.

Table 23: JOI $\Delta \mathrm{V}$ [m/s] for best Jupiter Capture at various RJ [24]

\begin{tabular}{l|ccccc}
\hline Flyby Sequences & 5 RJ & 4 RJ & 3 RJ & 2 RJ & 1 RJ \\
\hline \hline Unaided & 825 & 735 & 641 & 524 & 371 \\
Best Single & 556 & 526 & 483 & 416 & 308 \\
Best Double & 330 & 340 & 333 & 299 & 228 \\
Best Triple & 202 & 232 & 245 & 234 & 190 \\
Best Quad & N/A & N/A & N/A & 175 & 160 \\
\hline
\end{tabular}


These data further suggest that triple-satellite-aided captures may arguably provide the best opportunities for entry into the Jovian system. This is partially due to the non-existence of quadruple flyby sequences with reasonable perijove radii; a perijove radius of less than $3 \mathrm{RJ}$ would experience intense radiation from Jupiter, making only the first three columns of data worth considering. Of these, the triple-flyby sequence clearly provides substantial $\Delta \mathrm{V}$ reduction over other methods of capture.

\section{Adaptability}

Because the MATLAB model is all original script, written functionally and with an adaptive step size ode113 integrator, it can be easily modified to similarly examine different flyby sequences and even use low-thrust SEP. Such work has been examined in other existing software packages by Patrick and Lynam [39,40]. This method allows for alteration of all spacecraft initial conditions, allowing for various modes of interplanetary intercept, rather than the simple nearHohmann transfer employed to reach the initial state in this mission profile. By altering the interplanetary course, flyby encounter order, propulsion method and flyby safety margins, the capture could be further optimized for absolute minimal $\Delta \mathrm{V}$ or for a particular science schedule. This being said, further investigation would be required in order to determine if SEP would indeed be capable of performing the necessary corrections under the influence of the applied errors as currently defined. 


\section{Chapter 3: $8^{\text {th }}$ Annual Global Trajectory Optimisation Competition}

This chapter represents the problem provided for a competition and the methodology used by Dr. Alfred Lynam and his two students, Sean Patrick and the author, Alan Didion to solve the problem, as well as the relevant results. The problem was written by a team at NASA Jet Propulsion Laboratory (JPL) led by Anastassios Petropoulos, and teams were given one month to generate the best solution possible. Competing teams included engineers, research scientists, and students from 18 universities, companies, and government agencies from across the globe. The material comprising this chapter was originally submitted under the title: "Application of Resonant Orbits in Conjunction with Lunar Flybys in Low-Thrust Mission Design". More information, including the problem statement and competition history, can be located at the GTOC8 web portal. ${ }^{\mathrm{iii}}$ Much of the problem statement information contained herein is drawn from the given prompt for the competition [45].

The use of Very-Long-Baseline Interferometry (VLBI) in radio astronomy allows for superior angular resolution than other techniques. For the $8^{\text {th }}$ Global Trajectory Optimisation Competition (GTOC8), a group at JPL has organized a problem involving three spacecraft that are to be used for high resolution mapping of radio sources using VLBI. The problem focuses on optimizing low-thrust maneuvers to place these three spacecraft into simple formations so as to map the given radio sources. For this problem Lynam et al. have developed optimized trajectories through original scripts written in MATLAB.

In this project, the author was specifically tasked with designing and implementing all software to be used for testing ideas, simulating the mission, and sorting and scoring the resulting data. The author wrote a frame script and several functions from scratch in MATLAB

\footnotetext{
iii Global Trajectory Optimisation Competition Portal, [online], http://sophia.estec.esa.int/gtoc_portal/?page_id=560 (Accessed: 26 May 2015)
} 
and used these to develop and solve the trajectory ideas conceived and designed by Lynam and Patrick, which ranged from basic, short-term, low-fidelity simulations to visualize a mission concept to fully-functional, high-fidelity simulation and optimization programs to produce a finished product.

\section{A. Problem Description \& Scoring}

For this year's problem, the scenario began with three spacecraft, collocated in a circular orbit with an altitude of $400 \mathrm{~km}$ inside the ecliptic plane, positioned on the $\mathrm{x}$-axis at the starting epoch. The spacecraft each have two propulsion systems, a chemical propulsion system with a total $\Delta \mathrm{V}$ capacity of up to $3 \mathrm{~km} / \mathrm{s}$ and an $\mathrm{I}_{\mathrm{sp}}$ of $450 \mathrm{~s}$ and a low-thrust system with $\mathrm{I}_{\mathrm{sp}}$ of $5000 \mathrm{~s}$ and a maximum thrust of $0.1 \mathrm{~N}$. The chemical propulsion system may only be fired once, and it must be fired before the low-thrust system can be used; however, it need not use all $3 \mathrm{~km} / \mathrm{s}$ of available $\Delta \mathrm{V}$. In addition, the impulsive $\Delta \mathrm{V}$ must occur between MJD 58849.0 and 58880.0 and the mission had to end within three years. Each spacecraft could be up to $4000 \mathrm{~kg}$, of which 1890 kg was dry mass.

The purpose of this three-spacecraft constellation was to form a large triangle, the normal vector of which would represent a VLBI measurement boresight vector. Throughout the mission, the measurement vector would sweep across the sky, as governed by the movement of the satellite constellation, and its (geocentric) direction compared to a list of 420 given radio sources. If at any time the vector were to cross a source to within 0.1 degrees, a measurement would be counted. Measurements were required to have a 15 minute cool-down, to account for slewing and data recording, and were scored according to the procedure to follow. 


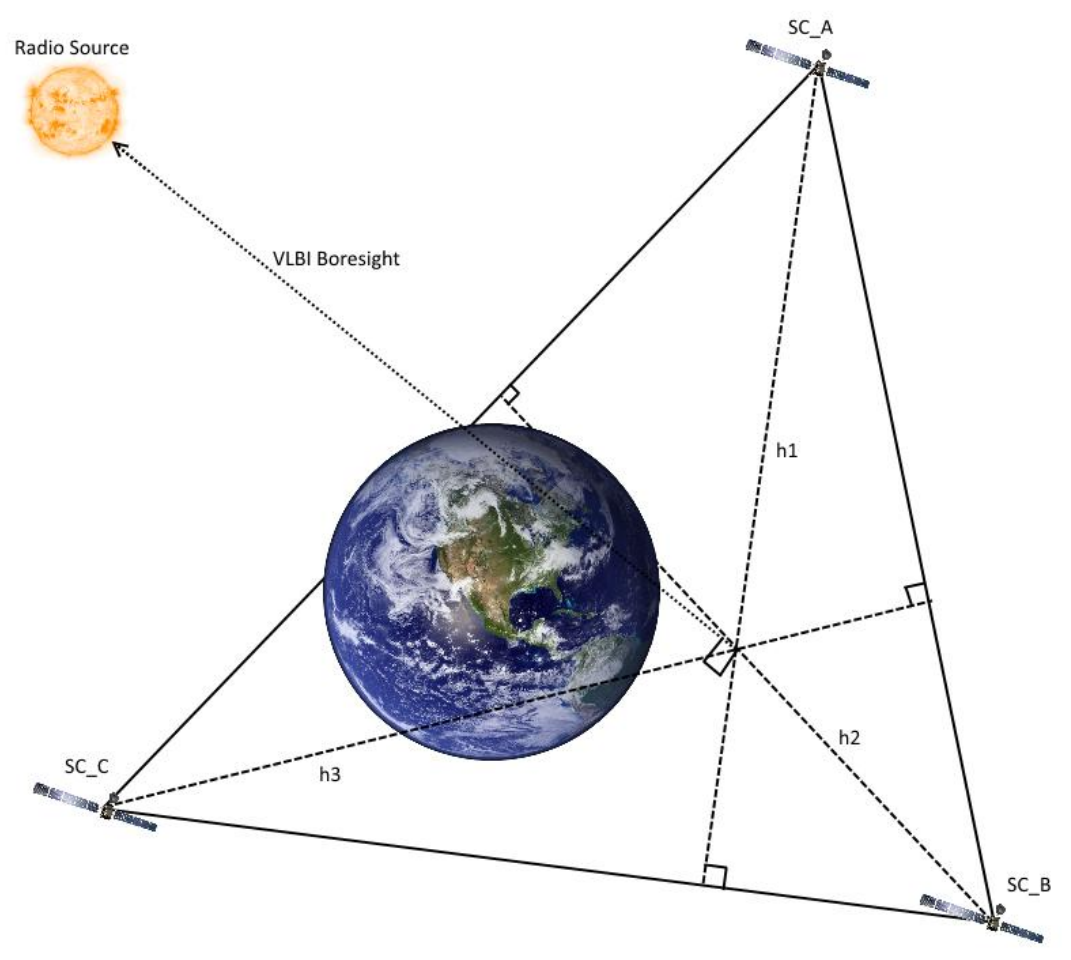

Figure 18: Diagram of an Example VLBI Measurement

As shown in Figure 18, one of the scoring parameters for a measurement was the maximum altitude, $\mathrm{h}$, of the VLBI triangle at the moment a measurement was counted. Recall, an altitude of a triangle is a distance from a vertex to a perpendicular side, and every triangle has three such quantities, which here correspond to the three spacecraft, A, B, and C. In order to be a valid measurement, the maximum altitude had to be greater or equal to $10,000 \mathrm{~km}$. The triangle normal, $\mathrm{n}$, is calculated using the geocentric radius vectors of the three spacecraft, as shown below in Equation 15. The $+/$ - reminds the investigator that the triangle can have a normal and an anti-normal vector and that both should be checked for source matches.

$$
\begin{gathered}
\vec{n}= \pm\left(\vec{r}_{B}-\vec{r}_{A}\right) \times\left(\vec{r}_{C}-\vec{r}_{A}\right) \\
\vec{s}=(\cos \delta * \cos \alpha) \hat{x}+(\cos \delta * \sin \alpha) \hat{y}+(\sin \delta) \hat{z}
\end{gathered}
$$


Equation 16 describes how source vectors, s, are calculated from the given data for declination and right ascension of the sources, denoted by $\delta$ and $\alpha$ respectively. These two equations can then be compared by taking the inverse cosine of the dot product between them. If this angle is less than 0.1 degrees, the match is counted as valid. Equation 17 below shows this operation.

$$
\varphi=\cos ^{-1}(\vec{n} \cdot \vec{s})
$$

Furthermore, repeat observations were rewarded with a repeat observation factor, $\mathrm{P}$, which has complex behavior as follows:

- First valid observation of a unique source: $\mathrm{P}=1$

- Second observation of a source, if the maximum altitude is at least three times the previous: $\mathrm{P}=3$, else: $\mathrm{P}=1$

- Third observation of a source, if the maximum altitude is at least six times that of the second: $\mathrm{P}=6$,

- if the maximum altitude is at least three times that of the second: $\mathrm{P}=3$, else: $\mathrm{P}=1$

- Fourth or greater observation of a source: $\mathrm{P}=0$

The scoring algorithm is detailed below in Equation 18, where $\mathrm{J}$ is the total score of the mission as a whole. $\mathrm{P}$ is the repeat observation factor and $\mathrm{h}$ is the VLBI triangle's maximum altitude, as previously described, and $\delta$ is simply the declination angle of the source being observed. 


$$
J=\sum_{\begin{array}{c}
\text { all } \\
\text { observations }
\end{array}} P h\left(0.2+\cos ^{2} \delta\right)
$$

Further rules governing the mission design included that the spacecraft orbital radii must all remain between $6578.14 \mathrm{~km}$ and $1000000.0 \mathrm{~km}$ and that the dynamical model include only gravitational influence from the Earth, neglecting the sun and moon, as will be shown in the next section. Any and all flybys of the moon were to be treated as instantaneous, modeled with patched-conics and assumed to occur at the position of the center of the moon; this will also be discussed in the following section.

\section{B. Given Conditions and Dynamical Model}

In addition to the given constraints on mission specifics, the simulation environment was rigidly prescribed as described herein. To begin, the necessary global physical constants were defined as in Table 24 below. This allowed all submitted solutions to be graded on the same metric, as these values can occasionally differ based on the preferred source of the author or over time through improved observations.

Table 24: Given Physical Constants

\begin{tabular}{lcrc}
\hline \multicolumn{1}{c}{ Parameter } & Symbol & \multicolumn{1}{c}{ Value } & Unit \\
\hline \hline Gravitational Parameter, Earth & $\mu$ & 398600.4329 & {$[\mathrm{~km} 3 / \mathrm{s} 2]$} \\
Gravitational Parameter, Moon & $\mu_{\mathrm{M}}$ & 4902.8006 & {$[\mathrm{~km} 3 / \mathrm{s} 2]$} \\
Radius, Earth & $\mathrm{R}_{\mathrm{E}}$ & 6378.14 & {$[\mathrm{~km}]$} \\
Radius, Moon & $\mathrm{R}_{\mathrm{M}}$ & 1737.5 & {$[\mathrm{~km}]$} \\
Gravitational Acceleration & $\mathrm{g}$ & 9.80665 & {$[\mathrm{~m} / \mathrm{s} 2]$} \\
Day & $\mathrm{N} / \mathrm{A}$ & 86400 & {$[\mathrm{~s}]$} \\
Year & $\mathrm{N} / \mathrm{A}$ & 365.25 & {$[\mathrm{day}]$} \\
\hline
\end{tabular}

\section{Dynamical Modeling}

The model to be used represents an Earth-centered inertial (J2000) coordinate frame, with numerical integration allowed for astrodynamical propagation, but patched conics only for lunar 
flybys. In this model, Earth is the central body and is fixed. The sun's gravitational influence is neglected as is that of the moon. Furthermore, the moon propagates dynamically (using Newtonian mechanics, not Keplerian elements or ephemerides). The set of Equations 19 below show the simple two-body acceleration of the moon around the earth, in terms of conventional Cartesian coordinates. The following Table 25 holds the moon's Keplerian elements as necessary to initialize the model, at the initial epoch of MJD 58849.0. These three equations are integrated each time-step throughout the simulation to describe the dynamical motion of the moon.

$$
\begin{gathered}
\ddot{x}+\mu \frac{x}{r^{3}}=0, \ddot{y}+\mu \frac{y}{r^{3}}=0, \ddot{z}+\mu \frac{z}{r^{3}}=0 \\
r=\sqrt{x^{2}+y^{2}+z^{2}}=\frac{a\left(1-e^{2}\right)}{1+e \cos \theta}
\end{gathered}
$$

Table 25: Moon Keplerian Orbital Elements

\begin{tabular}{cccc}
\hline Orbit Element & Symbol & Value & Unit \\
\hline \hline Semimajor-Axis & $\mathrm{a}$ & 383500.0 & {$[\mathrm{~km}]$} \\
Eccentricity & $\mathrm{e}$ & 0.04986 & {$[\mathrm{~N} / \mathrm{A}]$} \\
Inclination & $\mathrm{i}$ & 5.2586 & {$[\mathrm{deg}]$} \\
LAN & $\Omega$ & 98.0954 & {$[\mathrm{deg}]$} \\
Arg. Of Periapsis & $\omega$ & 69.3903 & {$[\mathrm{deg}]$} \\
Mean Anomaly & $\mathrm{M}_{0}$ & 164.35025 & {$[\mathrm{deg}]$} \\
\hline
\end{tabular}

The equations governing the propagation of the three spacecraft are similar to those of the moon, but include a thrust term, $T$, and instantaneous mass, $m$, of the individual spacecraft in the acceleration equation. Furthermore, the mass of the spacecraft is decremented according to the specific impulse of the SEP engines (recall, 5000 s) when firing. This can be seen below in Equations 21 and 22, similar to the previous equations in conventional Cartesian coordinates, though here the radius, $r$, is that of the spacecraft in question at the moment in question. Again, 
these three equations are integrated each time-step for each of the three spacecraft individually to fully describe their propagation through the system. These thrust values can be changed automatically by the script every time the propagation pauses for an important event or to take a measurement.

$$
\begin{gathered}
\ddot{x}+\mu \frac{x}{r^{3}}=\frac{T_{x}}{m}, \quad \ddot{y}+\mu \frac{y}{r^{3}}=\frac{T_{y}}{m}, \quad \ddot{z}+\mu \frac{z}{r^{3}}=\frac{T_{z}}{m} \\
\dot{m}=-\frac{T}{I_{S P} g}
\end{gathered}
$$

These equations are integrated in much the same way that the system from Chapter 2 is integrated. Important events such as timed maneuvers and the lunar rendezvous were detected within the loop, breaking the loop through the inclusion of an "events" function or by manually applying time constraints, depending on the specifics. For the majority of the mission, the integrator was set to stop at regular intervals to check for VLBI matches. These intervals were determined by examining the instantaneous geometry of the VLBI triangle and calculating the sweep-rate of the measurement vector. Time-steps were then selected to ensure that the measurement vector would sweep across the celestial sphere no more than its measurement resolution (0.1 deg) during any given propagation phase, and thus not miss a measurement opportunity.

\section{Patched-Conics for Lunar Flybys}

If a lunar flyby was to be used, and it was in this scenario, it was to occur instantaneously according to a patched-conic method. In such a method, the spacecraft's trajectory turns by a 
turning angle as described below. The v-infinity value is drawn automatically from the simulation at the moment of rendezvous and the flyby periapsis radius, $\mathrm{h}_{\mathrm{p}}$, is a design parameter.

$$
\sin \left(\frac{\delta_{t}}{2}\right)=\frac{\mu_{M} /\left(R_{M}+h_{p M}\right)}{v_{\infty}^{2}+\mu_{M} /\left(R_{M}+h_{p M}\right)}
$$

Furthermore, the v-infinity is to be conserved throughout the flyby, and the flyby periapsis must be higher than $50 \mathrm{~km}$. Additionally, the v-infinity had to be higher than $0.25 \mathrm{~km} / \mathrm{s}$; if all these conditions were met, the flyby would be considered valid.

\section{Impulsive Maneuvers}

As described in the competition rules, each spacecraft was to include an impulsive chemical engine and a low-thrust SEP engine. The mass decrement for low-thrust was handled very simply with each time-step, as described in Equation 22. The impulsive chemical engine, however, could only be fired once and its mass decrement was handled using a typical rocket equation. Again, the specific impulse for the chemical engine was $450 \mathrm{~s}$ and the $\Delta \mathrm{V}$ could be any value between 0 and $3 \mathrm{~km} / \mathrm{s}$, as chosen as a design parameter. This operation is described below, in Equation 24, in terms of mass after and before the maneuver ( + and - respectively) as an exponential function of the $\Delta \mathrm{V}$ design parameter.

$$
m\left(t^{+}\right)=m\left(t^{-}\right) \exp \left(-\frac{\Delta V}{g I_{S P}}\right)
$$

\section{Methodology}

To solve this, Lynam, et al. designed a trajectory in which one spacecraft (craft A, red in figures) was put into an elliptical, high inclination orbit. This is accomplished through the use of 
a maximum, purely prograde impulsive maneuver followed by continuous velocity direction low-thrust spiral and finally a gravity-assist of the moon with a 20,000 km flyby radius. After this flyby the spacecraft continued to thrust in the normal/anti-normal direction as appropriate, to raise the inclination further as the mission continued, switching when passing through the equatorial plane. The other two spacecraft (craft B and craft C, green and blue respectively) were put into elliptical orbits, apsides offset by 180 degrees from each other with each craft also offset 90 degrees from craft A's line of apsides. This was intended to ensure optimal radio source mapping geometry for this configuration. The maneuvers to put the craft into these orbits are timed such that the new periods have a resonance of an integer plus 0.5 with the starting orbit. This was a combined V-N (velocity-normal) maneuver, which used the remaining $\Delta \mathrm{V}$ to modify the inclination of both orbits up to a possible 10 degrees. The inclination component of the burns for craft B and craft $\mathrm{C}$ were opposite so as to place the two craft in the same plane.

Once the spacecraft constellation was constructed, the model was left to propagate until two years have passed. During this time, normal vectors were calculated and measurements were collected to determine source matches. A post-process sorting algorithm was employed to determine which matches are valid by applying the 15-day interval rule and striking the invalid measurements.

\section{Results}

A full, three year mission trajectory was successfully developed. This trajectory included the three spacecraft successfully being placed into the intended constellation and numerous valid measurements being recorded. Figure 19 below shows the first hours of the mission, wherein spacecraft A (red) loitered in the starting orbit until the time came to boost with maximum thrust into an orbit which later would allow for lunar rendezvous. At this point, it can be seen that 
spacecraft B (green) waited until it was offset 90 degrees from spacecraft A's periapsis and boosted with maximum impulsive thrust in a combined V-N maneuver described previously. As can be seen, the elliptical orbits of spacecraft B and C are 180 degrees opposed and in the same plane, 90 degrees to spacecraft $\mathrm{A}$.

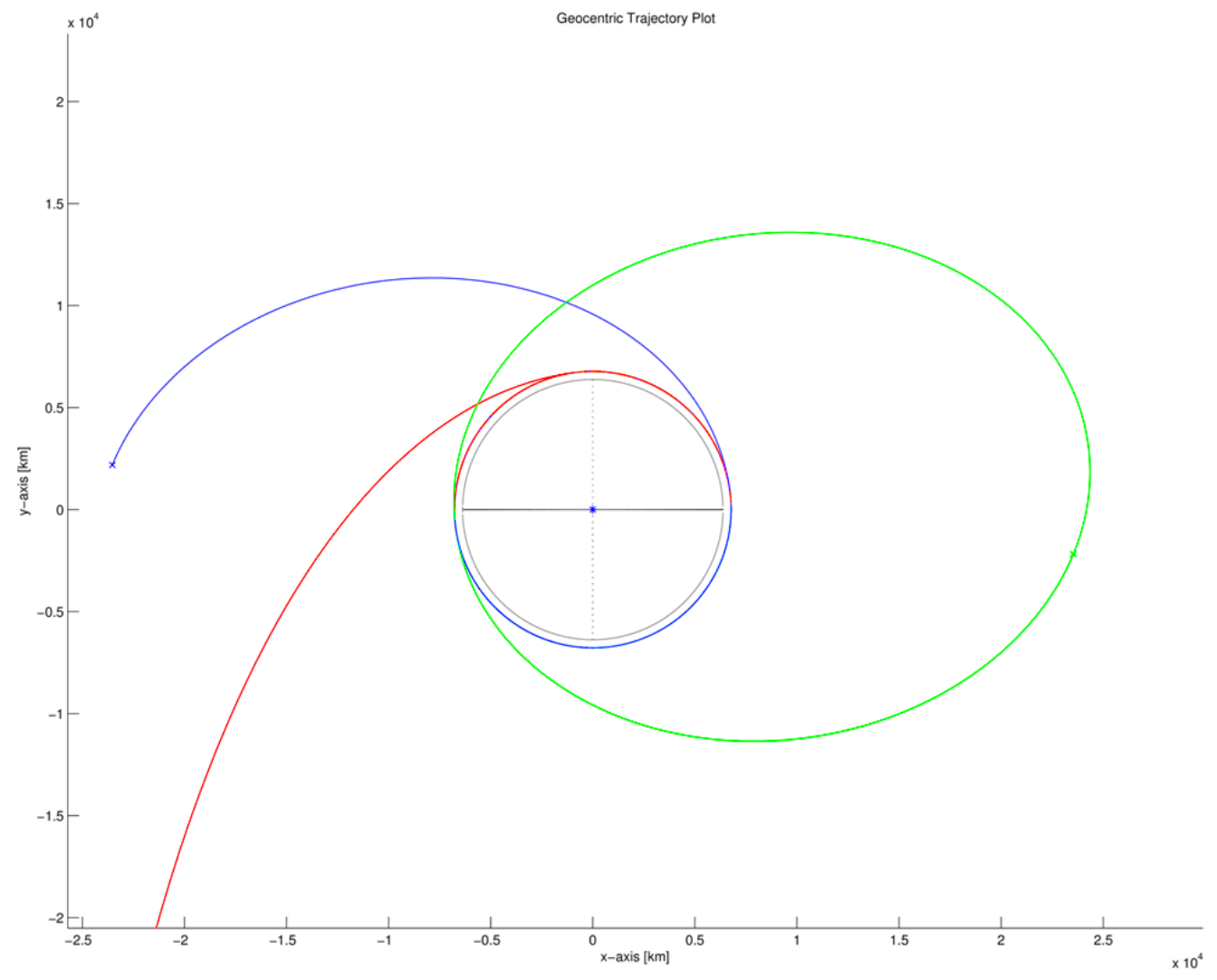

Figure 19: Geocentric X-Y Plot of Initial Constellation Architecture

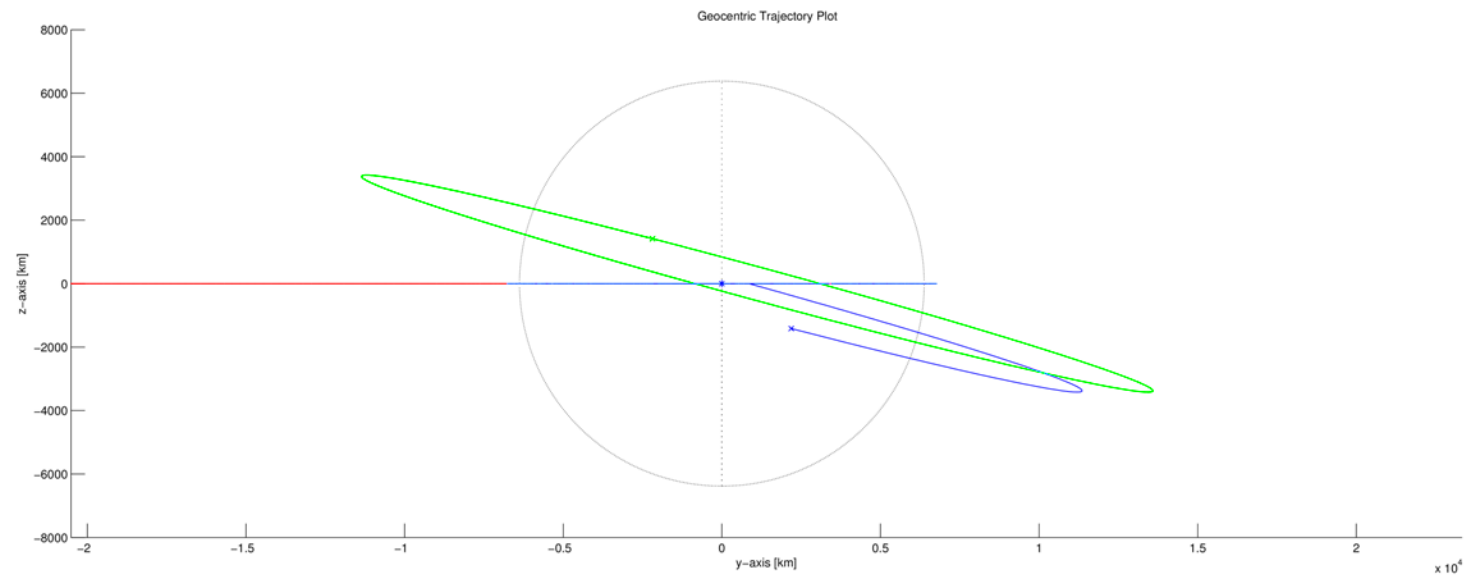

Figure 20: Geocentric Y-Z Plot of Initial Constellation Architecture 
A side view, shown in Figure 20, shows the ecliptic nature of the orbit of spacecraft A and the inclined resonant orbits of spacecraft B and C. Note: this is a side view with respect to Earth, and is not intended to be perpendicular to the plane of the orbits $\mathrm{B}$ and $\mathrm{C}$.

Immediately after completing the impulsive maneuver, spacecraft A begins firing its lowthrust engines to spiral out to the moon. This slow spiral can be seen in detail below in Figure 21, which is a top-down geocentric view showing the orbit of the moon and spacecraft A's lunar rendezvous. This lunar rendezvous was then performed as previously described. Note that the allowed $3 \mathrm{~km} / \mathrm{s}$ was not enough to impulsively reach lunar rendezvous, but $3.1 \mathrm{~km} / \mathrm{s}$ was more than enough to overshoot the moon. It is obvious that this was by design of the problem author in order to signify the necessity of a low-thrust outward spiral for a lunar flyby. This figure clearly displays the scale of the orbits of spacecraft B and C, as compared to that of spacecraft A and of the moon.

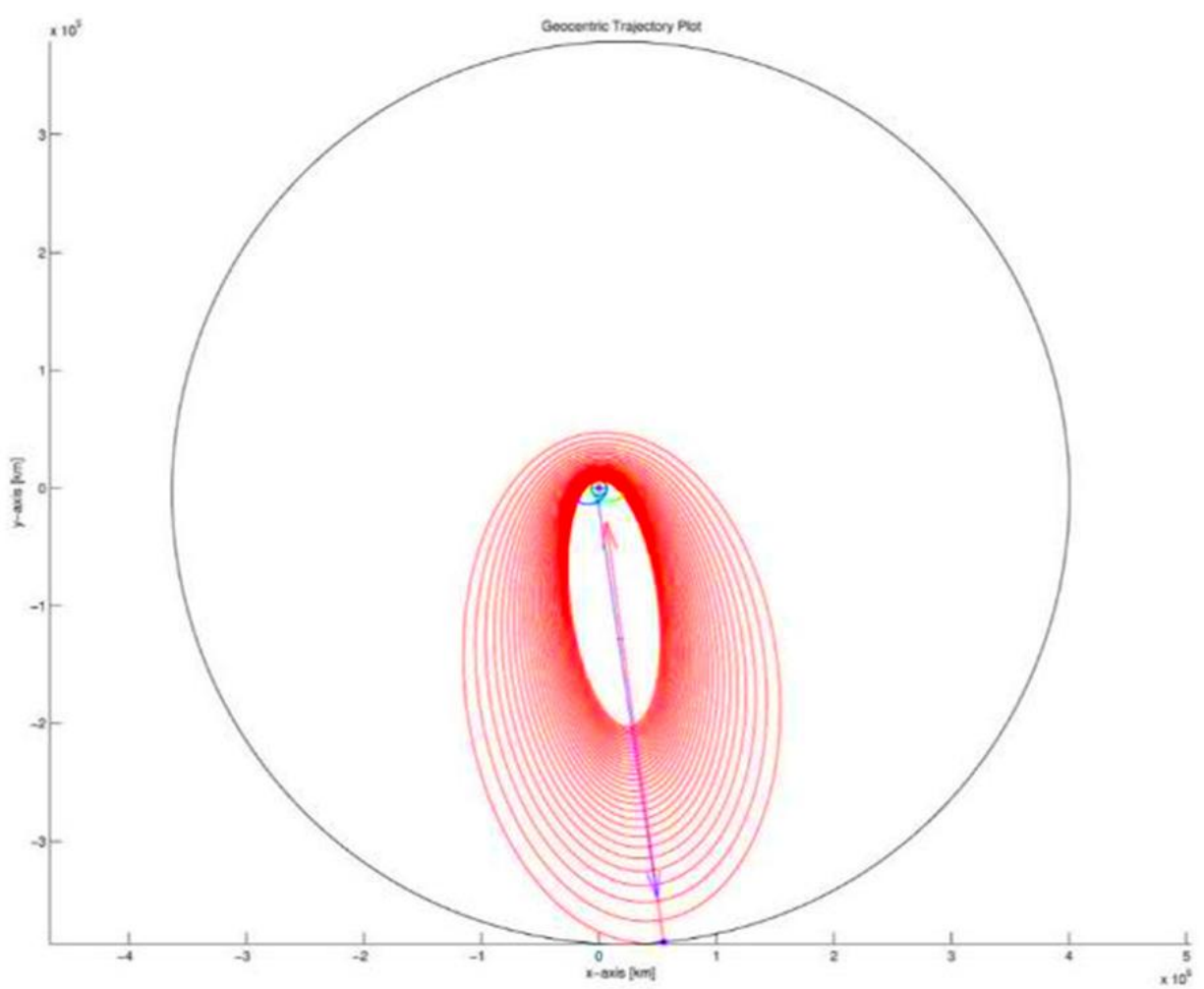

Figure 21: Geocentric X-Y Plot of Lunar Rendezvous, Zoomed to the Moon's Orbit 


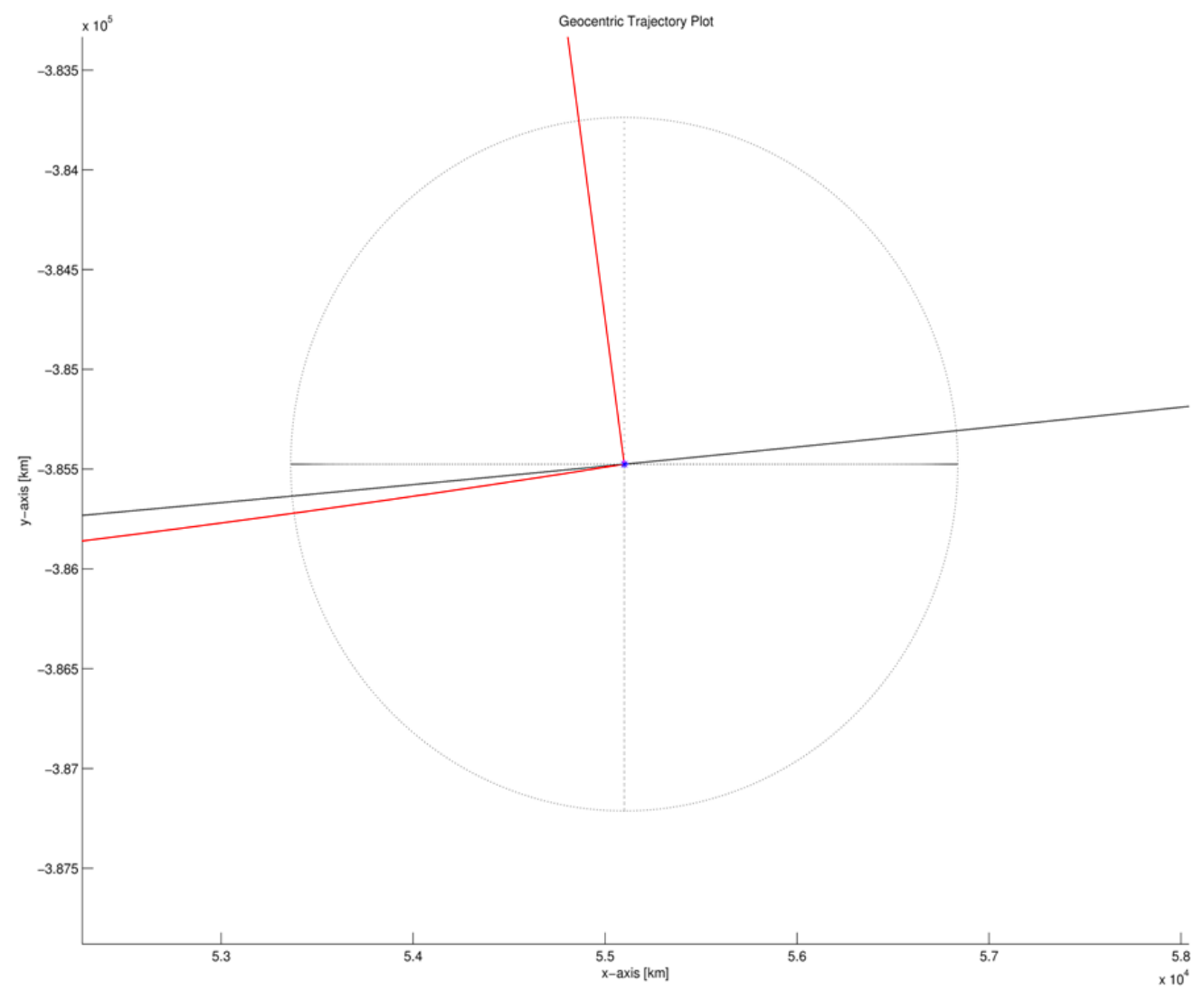

Figure 22: Selenocentric X-Y Plot of Lunar Rendezvous

Figure 22 is a closer view of the moment of spacecraft A's lunar rendezvous which verifies the requirement that lunar rendezvous be conducted at the exact center of the moon. The circle represents the surface of the moon and the dark and light solid lines represent the orbits of the moon and spacecraft A, respectively. Note that the trajectory of spacecraft A diverts sharply after the instantaneous flyby, as the spacecraft is propelled into a high-inclination orbit. The turn is actually not as severe as it appears here, and a more intuitive representation can be seen later in Figure 24.

Figure 23 is another top-down plot showing the eccentricity reduction of spacecraft A's orbit after the lunar flyby. At this point in the simulation, the spacecraft is now set to fire its low-thrust engines in whatever direction will increase inclination, until it runs out of fuel or the mission ends. This gives the VLBI vector a slow sweeping motion from high to low declination over 
time. Note in this figure that the VLBI measurement triangle is visible, and the measurement vector extends out from the intersection of altitudes.

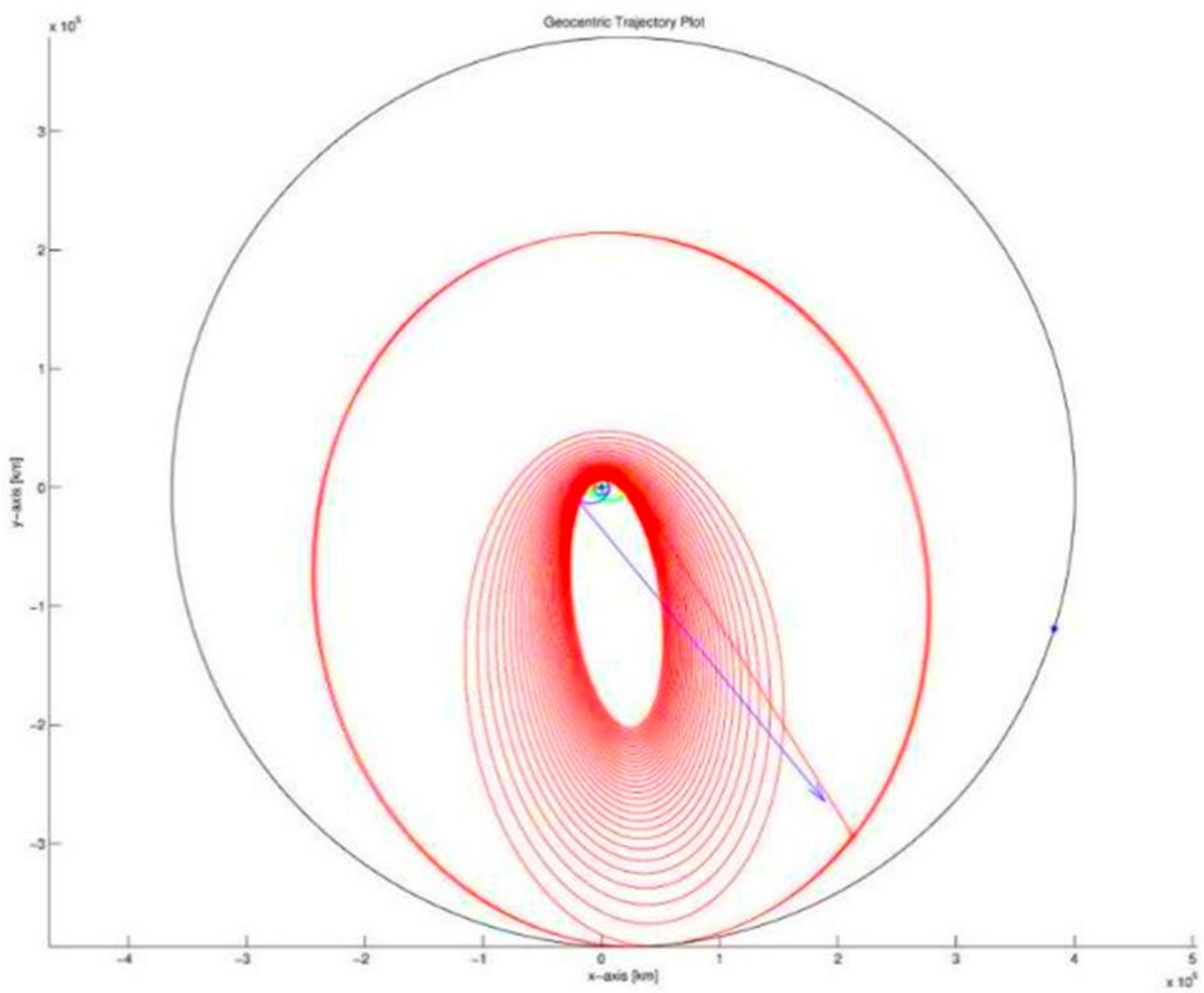

Figure 23: Geocentric X-Y Plot. 60 Days After Lunar Flyby

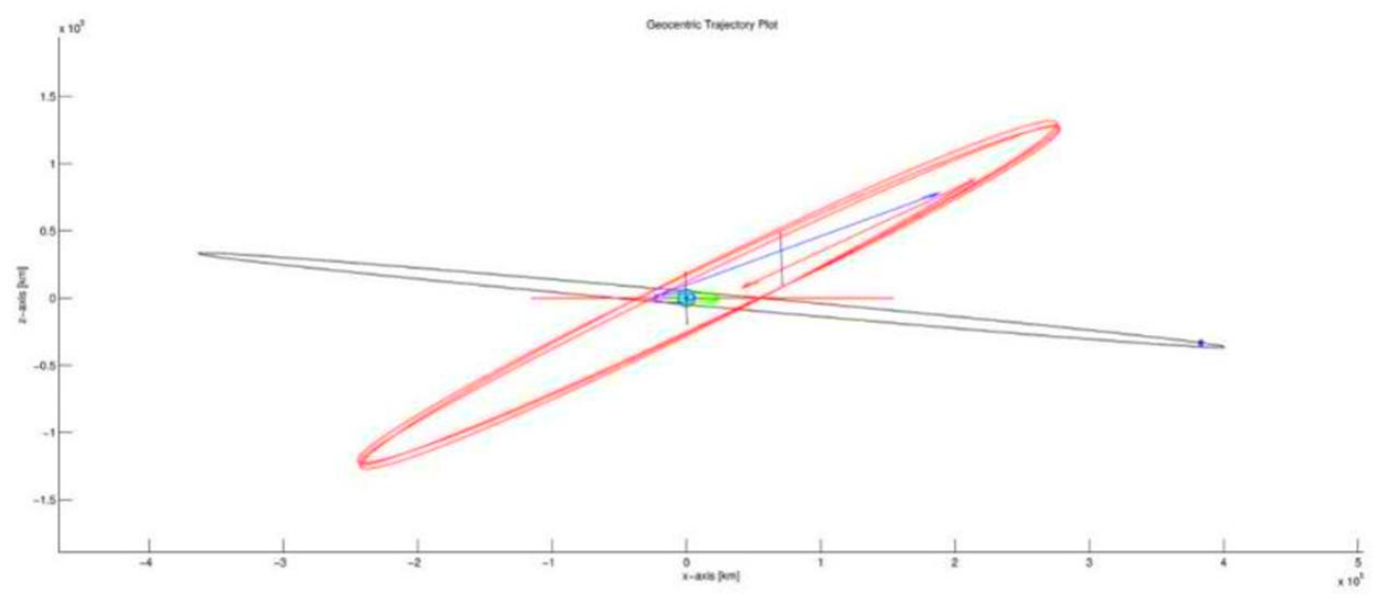

Figure 24: Geocentric X-Z Plot, 60 Days After Lunar Flyby 
Figure 24 shows the same information but again from a side view. In this view, the dramatic inclination change in spacecraft A's orbit provided by the lunar flyby is readily apparent. It is also apparent that direction in which the measurement points will sweep as spacecraft A continues to alter its inclination.

Meticulous track is kept of each event in the mission sequence. Table 26 below provides a mission time table with each important event recorded individually. Note that the initial boost from spacecraft $\mathrm{A}$ is not immediate, but timed in order to properly rendezvous with the moon as described. Additionally, the low-thrust SEP engines on spacecraft A begin to fire immediately after the impulsive maneuver is completed. The completed constellation shown in the previous few figures had nearly 800 days to propagate before the mission time limit is reached.

Table 26: Mission Timeline

\begin{tabular}{lrrl}
\hline Event & \multicolumn{1}{c}{ Time } & Time & Note \\
& MET [day] & MJD [day] & \\
\hline \hline Mission Start & 0.000 & 58849.000 & Given Epoch \\
SC A Impulse & 4.324 & 58853.324 & Maximum V-direction (3 km/s) \\
SC A SEP, V-direction & 4.324 & 58853.324 & Maximum V-direction $(0.1 \mathrm{~N})$ \\
SC B Impulse & 4.340 & 58853.340 & Combined V-N direction (norm <3 km/s) \\
SC C Impulse & 4.565 & 58853.565 & Combined V-N direction (norm <3 km/s) \\
SC A Targets Moon & 181.997 & 59030.997 & Optimizer, full VNC authority (norm $<0.1 \mathrm{~N})$ \\
SC A Moon Rendezvous & 186.997 & 59035.997 & Centimeter accuracy to lunar center \\
Lunar Flyby & 186.997 & 59035.997 & Instantaneous 20k km altitude flyby \\
SC A Long-term Inc-change & 186.997 & 59035.997 & Begin N/-N direction low-thrust \\
Mission End & 1095.750 & 59944.750 & Mission time limit reached \\
\hline
\end{tabular}

These specifics are for a mission with a $\mathrm{B} / \mathrm{C}$ orbit resonance of 3.5 to the period of the initial orbit. The rest of their remaining $\Delta \mathrm{V}$ is used to change the inclination of their orbits to about 10 degrees.

At mission end, each craft successfully expended all chemical propellant mass and spacecraft B and C had $2026.8 \mathrm{~kg}$ of mass remaining. Spacecraft A performed a longer post-flyby low- 
thrust maneuver arc, ending with $1914.7 \mathrm{~kg}$ of mass. With a dry mass of $1890.0 \mathrm{~kg}$, this leaves spacecraft A, B and C with $24.7 \mathrm{~kg}, 136.8 \mathrm{~kg}$, and $136.8 \mathrm{~kg}$ of fuel respectively at mission end. A full mass fuel budget is given in Table 27.

Over the course of the mission, the spacecraft constellation successfully matched 156 sources. After striking $4^{\text {th }}$ and higher repetitions and applying the 15-day measurement interval rule, 21 valid measurements were retained and submitted. When processed through the given scoring algorithm, the total score for this constellation is $181,688.6$ points. Note: after the trajectory files were processed by the scoring judges, the previously quoted score was found to be too low (the algorithm under-reported some scores) and the total score was adjusted upwards to $255,469.4$ points.

Table 27: Fuel Mass Budget

\begin{tabular}{lrrrr}
\hline & Time Start [MJD] & Time End [MJD] & Amount [kg] & Remaining [kg] \\
\hline \hline Spacecraft A & & & & \\
\hline Impulse & 58853.324 & N/A & 1973.191 & 2026.809 \\
Spiral & 58853.324 & 59030.997 & 31.264 & 1995.545 \\
Target Moon & 59030.997 & 59035.997 & 0.778 & 1994.767 \\
End of Mission & 59035.997 & 59944.750 & 80.064 & 1914.702 \\
\hline Spacecraft B & & & & \\
\hline Impulse & 58853.340 & N/A & 1973.191 & 2026.809 \\
\hline Spacecraft C & 5853.565 & N/A & 1973.191 & 2026.809 \\
\hline Impulse
\end{tabular}

\section{E. Conclusions}

The three-spacecraft constellation designed here benefits from a few key features. Namely, the resonance of the orbits of spacecraft $\mathrm{B}$ and $\mathrm{C}$ ensure that they are only ever as close as the sum of their periapsides (at least the diameter of Earth), ensuring a consistent altitude for the measurement triangle. Additionally, the flyby of spacecraft A with the moon allows for lowdeclination measurements, improving possible scores for the measured sources. By having 
spacecraft A continue normal/antinormal thrust after the lunar flyby, its inclination continues to change, sweeping lower and lower declinations for a more diverse set of source matches.

This arrangement, however, suffers from a few insufficiencies. Namely, the resonant orbits used in the results delivered here (factor of 3.5) have small, consistent size. Thus, the measurement triangle altitude is arguably too consistent; repeat observations are wasted, rather than accrue score multipliers. Furthermore, spacecraft A takes more than half a year, one sixth of the mission allowance, spiraling to the moon in the equatorial plane. It is possible that spacecraft A could have been inclined with the initial impulse, but this would have drastically lengthened the time necessary to reach the moon. Finally, multiple lunar flybys would possibly improve the equatorial searching capabilities of the constellation, but this was not examined because the postflyby normal thrust profile was deemed adequate.

Certainly, this design would benefit greatly from further optimization of the low-thrust maneuvering to achieve more repeat observations at various triangle altitudes. Unfortunately, such fine-tuning was time consuming and the code was computationally expensive, prohibiting more than only a few successful runs before the competition came to a close. 


\section{General Conclusions}

Throughout the course of this research, many questions were answered about triple-satelliteaided capture trajectories. In the first chapter, it was shown through use of the NASA GMAT software that triple-satellite-aided capture trajectories can work in a full dynamical simulation. Further, a suitable candidate was identified and optimized for the specific situation. The method developed can now easily be applied to other cases and proved definitively that the triple-flyby scenario provides significant $\Delta \mathrm{V}$ savings for Jovian insertion. However, questions were raised as to the sensitivity of the scenario to perturbations and navigational limitations.

In the second chapter, these questions were examined by applying numerous errors created by various sources including ephemeris inaccuracy, navigational inaccuracy, and maneuver execution limitations. These errors were applied in a pseudo-random fashion and repeated nearly two-thousand times to prove statistical significance. The results proved that even under less than ideal conditions, the previously formulated triple-flyby scenario is reasonably navigable with plausible near-term methods. This brought the scenario into the "real world", putting to rest the doubts raised by the ideal model used in the first chapter's GMAT model.

In the third chapter, the author and his team expanded their astrodynamics experience and demonstrated their ability to solve complex astrodynamics problems in short spans of time. A complex MATLAB script was written from scratch in short order that created a reasonable solution to the GTOC8 problem. The team demonstrated its ability to generate a creative solution while working within tight constraints and rules. The final solution generated could benefit from further optimization, but the fact that a proper solution was found in the given time is considered a mark of success. 
This thesis as a whole details the entirety of the author's graduate research experience and his demonstrated ability to understand, identify, and formulate solutions to complex astrodynamical problems. It further demonstrates the academic and professional interests of the author in the space industry and particularly in complex trajectory design, optimization, and navigation. The methods contained herein include existing software and user-created scripts and hierarchies of functions. 


\section{References}

[1] Potts, C. L., Wilson, M. G., "Maneuver Design for the Galileo VEEGA Trajectory," AAS/AIAA Astrodynamics Specialist Conference, AAS Paper No 93-566, Victoria, BC, Canada, August 1993.

[2] Barber, T. J., Krug, F. A., Froidevaux, B. M., "Initial Galileo Propulsion System In-flight Characterization," AIAA/SAE/ASME/ASEE $29^{\text {th }}$ Joint Propulsion Conference and Exhibit, AIAA Paper No: 93-2117, Monterrey, CA, June 1993.

[3] Wilson, M. G., Potts, C. L., Mase, R. A., Halsell, C. A., Byrnes, D. V., "Maneuver Design

for Galileo Jupiter Approach and Orbital Operations," $12^{\text {th }}$ International Symposium for Spaceflight Dynamics, Darmstadt, Germany, June 1997.

[4] Haw, R. J., Antreasian, P. G., McElrath, T. P., and Lewis, G. D., "Galileo Prime Mission Navigation," Journal of Spacecraft and Rockets, Vol. 37, No. 1, 2000, pp. 56-63.

[5] Goodson, T. D., Gray, D. L., Hahn, Y., Peralta, F., "Cassini Maneuver Experience: Finishing Inner Cruise," AAS/AIAA Spaceflight Mechanics Meeting, AAS Paper No 00-167, Clearwater, FL, January 2000.

[6] Roth, D., Antreasian, P., Bordi, J., Criddle, K., Ionesescu, R., Jacobson, R., Jones, J., Meed, M. C., Roundhill, I., Stauch, J., "Cassini Orbit Reconstruction from Jupiter to Saturn," AAS/AIAA Astrodynamics Specialist Conference, AIAA Paper No 05-311, Lake Tahoe, CA, August 2005.

[7] Ballard, C. G., Attieta, J., Hahn, Y., Stumpf, P. W., Wagner, S. V., Williams, P. N., "Cassini Maneuver Experience: Ending the Equinox Mission," AIAA/AAS Astrodynamics Specialist Conference, Toronto, ON, Canada, August 2010. 
[8] Williams, P.N., Ballard, C. G., Gist, E. M., Goodson, T. D., Hahn, Y., Stumpf, P. W., Wagner, S. V., "Flight Path Control Design for the Cassini Equinox Mission," AAS/AIAA Spaceflight Mechanics Meeting, Savannah, GA, February 2009.

[9] Anderson, J. D., Lau, E. L., Sjogren, W. L., Schubert, G., and Moore, W. B., “Gravitational Constraints on the Internal Structure of Ganymede,” Nature, Vol. 384, 1996, pp. $541-543$.

[10] Anderson, J. D., Jacobson, R. A., McElrath, T. P. Moore, W. B., Schubert, G., and Thomas P. C., "Shape, Mean Radius, Gravity Field, and Interior Structure of Callisto," Icarus, Vol. 153, 2001.

[11] Anderson, J. D., Jacobson, R. A., Lau, E. L., Moore, W. B., Schubert, G., “Io’s gravity field and interior structure," Journal of Geophysical Research, Vol. 106, 2001.

[12] Anderson, J. D., Schubert, G., Jacobson, R. A., Lau, E. L., Moore, W. B., and Sjogren, W. L., "Europa's differentiated internal structure: Inferences from four Galileo encounters," Science, Vol. 281, 1998.

[13] Longman, R. W., “Gravity Assist from Jupiter's Moons for Jupiter-Orbiting Space Missions,” The RAND Corp., Santa Monica, CA, 1968.

[14] Longman, R. W., Schneider, A. M. “Use of Jupiter's Moons for Gravity Assist,” Journal of Spacecraft and Rockets, Vol. 7, 1970, pp. 570-576.

[15] Cline, J. K., “Satellite Aided Capture”, Celestial Mechanics, Vol. 19, 1979, pp. 405-415.

[16] Nock, K. T., Uphoff, C., "Satellite Aided Orbit Capture," AAS/AIAA Astrodynamics Specialist Conference, AAS Paper No 79-165, Provincetown, MA, June 1979. 
[17] Johannesen, J. R., D’Amario, L. A., "Europa Orbiter Mission Trajectory Design," AAS/AIAA Astrodynamics Specialist Conference, AAS Paper No 99-330, Girdwood, AK, August 1999.

[18] Yam, C. H., "Design of Missions to the Outer Planets and Optimization of Low-thrust, Gravity-assist Trajectories via Reduced Parameterization,” PhD. Dissertation, Purdue University, 2008.

[19] Landau, D. F., Strange, N., Lam, T., "Solar Electric Propulsion with Satellite Flyby for Jovian Capture,” AAS/AIAA Spaceflight Mechanics Conference, San Diego, CA, February 2010.

[20] Strange, N., Landau, D. F., Hofer, R., Snyder, J. S., Randolph, Campagnola, S., Szabo, J., Pote, B., "Solar Electric Propulsion Gravity-Assist Tours for Jupiter Missions," AIAA/AAS Astrodynamics Specialist Conference, AIAA Paper No 2012-4518, Minneapolis, MN, August 2012.

[21] Lynam, A. E., Kloster, K. W., Longuski, J. M., “An Assessment of Multiple-SatelliteAided Capture," AAS/AIAA Astrodynamics Specialist Conference, AAS Paper No 09-424, Pittsburgh, PA, August 2009.

[22] Lynam, A. E., Kloster, K. W., Longuski, J. M., "Multiple-satellite-aided Capture Trajectories at Jupiter using the Laplace Resonance," Celestial Mechanics and Dynamical Astronomy, Vol. 109, 2011, pp. 59-84.

[23] Lynam, A. E., Longuski, J. M., "Interplanetary Trajectories for Multiple-satellite-aided Capture at Jupiter," Journal of Guidance, Control, and Dynamics, Vol. 34, 2001, 1485-1494.

[24] Lynam, A. E., Longuski, J. M., "Preliminary Analysis for the Navigation of Multiplesatellite-aided capture at Jupiter," Acta Astronautica, Vol. 70, 2012, pp. 33-43. 
[25] Lynam, A. E., "Broad-search Algorithms for the Spacecraft Trajectory Design of Callisto-Ganymede-Io Triple Flyby Sequences from 2024 to 2040, Part I: Heuristic Pruning of the Search Space," Acta Astronautica, Vol. 94, Issue 1, January-February 2014, pp. 246-252.

[26] Lynam, A. E., "Broad-search Algorithms for the Spacecraft Trajectory Design of Callisto-Ganymede-Io Triple Flyby Sequences from 2024 to 2040, Part II: Lambert Pathfinding and Trajectory Solutions," Acta Astronautica, Vol. 94, Issue 1, January-February 2014, pp. 253261.

[27] Lynam, A. E., "Broad-Search Algorithms for Finding Triple- and Quadruiuple-SatelliteAided Captures at Jupiter from 2020-2080," Celestial Mechanics and Dynamical Astronomy, Vol. 121, Issue 4, pp. 347-363.

[28] Planetary Satellite Physical Parameters, JPL Solar System Dynamics, [online], http://ssd.jpl.nasa.gov/?sat_phys_par (Accessed: 6 January 2014).

[29] Thomas, P. C., Davies, M. E., Colvin, T. R., Oberst, J., Schuster, P., Neukum, G., Carr, M. H., McEwen, A., Schubert, G., Belton, M. J. S., and the Galileo Imaging Team 1998, “The Shape of Io from Galileo Limb Measurements," Icarus 135, 175-180.

[30] Anderson, J. D., Jacobson, R. A., Lau, E. L., Moore, W. B., Olsen, O., Schubert, G., and Thomas, P. C. 2000. "Shape, Mean Radius, Gravity Field and Interior Structure of Ganymede," Bulletin of the American Astronomical Society 33(3), 1101.

[31] Anderson, J. D., Jacobson, R.A., McElrath, T. P., Schubert,G., Moore, W.B., and Thomas, P. C. 2000a. "Shape, Mean Radius, Gravity Field and Interior Structure of Callisto," Icarus 153, 157-161. 
[32] Donahue, B. B., Klaus, K., “The Space Launch System Capabilities for Beyond Earth Missions," 50 th Annual AIAA/ASME/SAE/ASEE Joint Propulsion Conference, Cleveland, Ohio, July 2014, doi: 10.2514/6.2014-4021.

[33] Broucke, R. A., "The Celestial Mechanics of Gravity Assist," Proceedings of the AIAA/AAS Astrodynamics Specialist Conference, AIAA Paper 88-4220-CP, Minneapolis, MN, August 1988.

[34] Laplace, P. S., The System of the World, W. Flint, London, 1809.

[35] Strom, R. G., Terrile, R. J., Hansen, C., Masursky, H., "Volcanic eruption plumes on Io," Nature, Vol. 280, August 1979, pp. 733-736.

[36] Plainaki, C., Milillo, A., Massetti, S., Mura, A., Jia, X., Orsini, S., Mangano, V., De Angelis, E., Rispoli, R., "The $\mathrm{H} 2 \mathrm{O}$ and $\mathrm{O} 2$ exospheres of Ganymede: The result of a complex interaction between the jovian magnetospheric ions and the icy moon," Icarus, Vol. 245, January 2015, pp. 306-319.

[37] Didion, A. M., and Lynam, A. E., "Impulsive Trajectories from Earth to Callisto-IoGanymede Triple Flyby Capture at Jupiter," AAS/AIAA Astrodynamics Specialist Conference, AIAA Paper 2014-4106, San Diego, California, August 2014.

[38] Didion, A. M., and Lynam, A. E., "Impulsive Trajectories from Earth to Callisto-IoGanymede Triple Flyby Jovian Capture," Journal of Spacecraft and Rockets, Vol. 52, No. 3, 2015, pp. 746-753.

[39] Patrick, S. K., and Lynam, A. E., “Optimal SEP Trajectories from Earth to Jupiter with Triple Flyby Capture," AAS/AIAA Astrodynamics Specialist Conference, AIAA Paper 20144218, San Diego, CA, August 2014. 
[40] Patrick, S. K., and Lynam, A. E., "High-Fidelity Low-thrust SEP Trajectories From Earth to Jupiter Capture," AAS/AIAA Astrodynamics Specialist Conference, AAS Paper AAS-15-609, Vail, CO, August 2015.

[41] Wagner, S. V., and Goodson, T. D., "Execution-Error Modeling and Analysis of the Cassini-Huygens Spacecraft Through 2007," Proceedings of the AAS/AIAA Space Flight Mechanics Meeting, AAS Paper 08-113, Galveston, TX, January 2008.

[42] Vallado, D. A., and McClain, W.D., Fundamentals of Astrodynamics and Applications, Netherlands: Springer Science and Business Media, $2^{\text {nd }}$ ed., 2001.

[43] Curkendall, D. W., and Border, J. S., "Delta-DOR: The One-Nanoradian Navigation Measurement System of the Deep Space Network - History, Architecture, and Componentry," IPN Progress Report, Issue 42-19, May 2013.

[44] Didion, A. M., and Lynam, A. E., "Guidance and Navigation of a Callisto-Io-Ganymede Triple Flyby Jovian Capture," AAS/AIAA Astrodynamics Specialist Conference, AAS Paper AAS 15-624, Vail, CO, August 2015.

[45] Petropoulos, A. E., "Problem Description for the $8^{\text {th }}$ Global Trajectory Optimisation Competition," [online], http://sophia.estec.esa.int/gtoc_portal/wp-content/uploads/2015/05/gtoc8_problem_stmt.pdf (Accessed: 26 May 2015). 